\title{
LOCAL EXISTENCE OF SOLUTIONS OF A FREE BOUNDARY PROBLEM FOR EQUATIONS OF COMPRESSIBLE VISCOUS HEAT-CONDUCTING FLUIDS
}

Abstract. The local existence and the uniqueness of solutions for equations describing the motion of viscous compressible heat-conducting fluids in a domain bounded by a free surface is proved. First, we prove the existence of solutions of some auxiliary problems by the Galerkin method and by regularization techniques. Next, we use the method of successive approximations to prove the local existence for the main problem.

1. Introduction. This paper is concerned with the local motion of a drop of a viscous compressible heat-conducting fluid. Let $\Omega_{t} \subset \mathbb{R}^{3}$ be a bounded domain of the drop at time $t$. Let $v=v(x, t)\left(v=\left(v_{1}, v_{2}, v_{3}\right)\right)$ be the velocity of the fluid, $\varrho=\varrho(x, t)$ the density, $\theta=\theta(x, t)$ the temperature, $f=f(x, t)$ the external force field per unit mass, $r=r(x, t)$ the heat sources per unit mass, $\bar{\theta}=\bar{\theta}(x, t)$ the heat flow per unit surface, $p=p(\varrho, \theta)$ the pressure, $c_{v}=c_{v}(\varrho, \theta)$ the specific heat at constant volume, $\mu$ and $\nu$ the constant viscosity coefficients, $\kappa$ the constant coefficient of heat conductivity, and $p_{0}$ the external (constant) pressure. Then the motion of the drop is described by the following system of equations (see [3], Chs. 2 and 5):

$$
\begin{array}{ll}
\varrho\left[v_{t}+(v \cdot \nabla) v\right]-\operatorname{div} \mathbf{T}(v, p)=\varrho f & \text { in } \widetilde{\Omega}^{T}, \\
\varrho_{t}+\operatorname{div}(\varrho v)=0 & \text { in } \widetilde{\Omega}^{T},
\end{array}
$$

$$
\begin{aligned}
\varrho c_{v}\left(\theta_{t}+v \cdot \nabla \theta\right)-\kappa \Delta \theta+\theta p_{\theta} \operatorname{div} v \\
\quad-\frac{\mu}{2} \sum_{i, j=1}^{3}\left(v_{i x_{j}}+v_{j x_{i}}\right)^{2}-(\nu-\mu)(\operatorname{div} v)^{2}=\varrho r \quad \text { in } \widetilde{\Omega}^{T},
\end{aligned}
$$

1991 Mathematics Subject Classification: 35A05, 35R35, 76N10.

Key words and phrases: free boundary, compressible viscous heat-conducting fluids, local existence. 


$$
\begin{aligned}
& \mathbf{T} \cdot \bar{n}=-p_{0} \bar{n} \\
& \text { on } \widetilde{S}^{T} \text {, } \\
& v \cdot \bar{n}=-\phi_{t} /|\nabla \phi| \quad \text { on } \widetilde{S}^{T} \text {, } \\
& \frac{\partial \theta}{\partial n}=\bar{\theta} \quad \text { on } \widetilde{S}^{T}, \\
& \left.\varrho\right|_{t=0}=\varrho_{0},\left.\quad v\right|_{t=0}=v_{0},\left.\quad \theta\right|_{t=0}=\theta_{0} \quad \text { in } \Omega,
\end{aligned}
$$

where $\widetilde{\Omega}^{T}=\bigcup_{t \in(0, T)} \Omega_{t} \times\{t\}, \widetilde{S}^{T}=\bigcup_{t \in(0, T)} S_{t} \times\{t\}, S_{t}=\partial \Omega_{t}, \phi(x, t)=0$ describes $S_{t}, \bar{n}$ is the unit outward vector normal to the boundary, i.e. $\bar{n}=\nabla \phi /|\nabla \phi|, \Omega=\left.\Omega_{t}\right|_{t=0}=\Omega_{0}$. By $\mathbf{T}=\mathbf{T}(v, p)$ we denote the stress tensor of the form

$$
\mathbf{T}(v, p)=\left\{T_{i j}\right\}_{i, j=1,2,3}=\left\{-p \delta_{i j}+D_{i j}(v)\right\}_{i, j=1,2,3},
$$

where

$$
\mathbb{D}(v)=\left\{D_{i j}(v)\right\}_{i, j=1,2,3}=\left\{\mu\left(v_{i x_{j}}+v_{j x_{i}}\right)+(\nu-\mu) \delta_{i j} \operatorname{div} v\right\}_{i, j=1,2,3}
$$

is the deformation tensor. Moreover, in view of the thermodynamic considerations we assume that $c_{v}>0, \kappa>0, \nu>\frac{1}{3} \mu>0$.

Let the domain $\Omega$ be given. Then by $(1.1)_{5}$,

$$
\Omega_{t}=\left\{x \in \mathbb{R}^{3}: x=x(\xi, t), \xi \in \Omega\right\},
$$

where $x=x(\xi, t)$ is the solution of the Cauchy problem

$$
\frac{\partial x}{\partial t}=v(x, t),\left.\quad x\right|_{t=0}=\xi \in \Omega, \quad \xi=\left(\xi_{1}, \xi_{2}, \xi_{3}\right) .
$$

Integrating (1.3) we obtain the following relation between the Eulerian $x$ and Lagrangian $\xi$ coordinates of the same fluid particle:

$$
x=\xi+\int_{0}^{t} u\left(\xi, t^{\prime}\right) d t^{\prime} \equiv X_{u}(\xi, t),
$$

where $u(\xi, t)=v\left(X_{u}(\xi, t), t\right)$. Moreover, by $(1.1)_{5}, S_{t}=\{x: x=x(\xi, t)$, $\xi \in S=\partial \Omega\}$.

By the continuity equation $(1.1)_{2}$ and the kinematic condition $(1.1)_{5}$ the total mass is conserved, i.e.

$$
\int_{\Omega_{t}} \varrho(x, t) d x=\int_{\Omega} \varrho_{0}(\xi) d \xi=M,
$$

where $M$ is a given constant.

The aim of the paper is to show the local existence theorem for problem (1.1). In order to prove the local-in-time existence of solutions of (1.1) we 
rewrite it in Lagrangian coordinates as follows:

$$
\begin{array}{ll}
\eta u_{t}-\operatorname{div}_{u} \mathbf{T}_{u}(u, p)=\eta g & \text { in } \Omega^{T}=\Omega \times(0, T), \\
\eta_{t}+\eta \operatorname{div}_{u} u=0 & \text { in } \Omega^{T}, \\
\eta c_{v}(\eta, \gamma) \gamma_{t}-\kappa \nabla_{u}^{2} \gamma+\gamma p_{\gamma}(\eta, \gamma) \operatorname{div}_{u} u & \\
\quad-\frac{\mu}{2} \sum_{i, j=1}^{3}\left(\xi_{x_{i}} \cdot \nabla_{\xi} u_{j}+\xi_{x_{j}} \cdot \nabla_{\xi} u_{i}\right)^{2} & \\
\quad-(\nu-\mu)\left(\operatorname{div}_{u} u\right)^{2}=\eta k & \text { in } \Omega^{T}, \\
\mathbf{T}_{u}(u, p) \cdot \bar{n}_{u}=-p_{0} \bar{n}_{u} & \text { on } S^{T}=S \times(0, T), \\
\bar{n}_{u} \cdot \nabla_{u} \gamma=\bar{\gamma} & \text { on } S^{T}, \\
\left.\eta\right|_{t=0}=\varrho_{0},\left.\quad u\right|_{t=0}=v_{0},\left.\quad \gamma\right|_{t=0}=\theta_{0} & \text { in } \Omega,
\end{array}
$$

where $\eta(\xi, t)=\varrho\left(X_{u}(\xi, t), t\right), \gamma(\xi, t)=\theta\left(X_{u}(\xi, t), t\right), p=p(\eta, \gamma), g(\xi, t)=$ $f\left(X_{u}(\xi, t), t\right), k(\xi, t)=r\left(X_{u}(\xi, t), t\right), \bar{\gamma}(\xi, t)=\bar{\theta}\left(X_{u}(\xi, t), t\right), \nabla_{u}=\xi_{i x} \partial_{\xi i}$, $\mathbf{T}_{u}(u, p)=-p I+\mathbb{D}_{u}(u), I=\left\{\delta_{i j}\right\}_{i, j=1,2,3}$ is the unit matrix, $\mathbb{D}_{u}(u)=$ $\left\{D_{u i j}(u)\right\}_{i, j=1,2,3}=\left\{\mu\left(\partial_{x_{i}} \xi_{k} \partial_{\xi_{k}} u_{j}+\partial_{x_{j}} \xi_{k} \partial_{\xi_{k}} u_{i}\right)+(\nu-\mu) \delta_{i j} \operatorname{div}_{u} u\right\}, \operatorname{div}_{u} u$ $=\nabla_{u} \cdot u=\partial_{x_{i}} \xi_{k} \partial_{\xi_{k}} u_{i}, \operatorname{div}_{u} \mathbf{T}(u, p)=\left\{\partial_{x_{j}} \xi_{k} \partial_{\xi_{k}} T_{u i j}(u, p)\right\}_{i=1,2,3}\left(\partial_{x_{i}} \xi_{k}\right.$ are the elements of the matrix $\xi_{x}$ which is inverse to $\left.x_{\xi}=I+\int_{0}^{t} u_{\xi}\left(\xi, t^{\prime}\right) d t^{\prime}\right)$ and summation over repeated indices is assumed.

Let $S_{t}$ be determined (at least locally) by the equation $\phi(x, t)=0$. Then $S$ is described by $\left.\phi(x(\xi, t), t)\right|_{t=0} \equiv \widetilde{\phi}(\xi)=0$. Thus, we have

$$
\bar{n}_{u}=\bar{n}\left(X_{u}(\xi, t), t\right)=\left.\frac{\nabla_{x} \phi(x, t)}{\left|\nabla_{x} \phi(x, t)\right|}\right|_{x=X_{u}(\xi, t)} \quad \text { and } \quad \bar{n}_{0}=\bar{n}_{0}(\xi)=\frac{\nabla_{\xi} \widetilde{\phi}(\xi)}{\left|\nabla_{\xi} \widetilde{\phi}(\xi)\right|} .
$$

The proof of the existence of solutions of problem (1.4) is divided into a few steps. First, we examine the auxiliary problem (3.1) and the problem

$$
\begin{array}{ll}
\eta c_{v}(\eta, \beta) \gamma_{t}-\kappa \nabla_{\xi}^{2} \gamma & \\
\quad=K+\frac{\mu}{2} \sum_{i, j=1}^{3}\left(\xi_{x_{i}} \cdot \nabla_{\xi} w_{j}+\xi_{x_{j}} \cdot \nabla_{\xi} w_{i}\right)^{2} & \text { in } \Omega^{T}, \\
\bar{n} \cdot \nabla_{\xi} \gamma=\bar{\gamma} & \text { on } S^{T}, \\
\left.\gamma\right|_{t=0}=\theta_{0} & \text { in } \Omega,
\end{array}
$$

where $\eta>0, \beta>0$ and $w$ are given functions, $\xi_{x_{i}}=\xi_{x_{i}}(w)$. We prove the existence of solutions of problems (3.1) and (1.5) by the Galerkin method and by some regularization techniques.

Next, by using the Schauder-Tikhonov fixed point theorem we obtain the local existence of solutions of problems (3.40) and (3.76) (see Lemmas 3.5 and 3.6). 
Finally, applying the method of successive approximations we prove the local existence and the uniqueness of a solution $(u, \gamma, \eta)$ of problem (1.4) such that $u, \gamma \in \mathcal{A}_{T}, \eta \in \mathcal{B}_{T}$, where $T \leq T_{*}, T_{*}>0$ is a certain constant; $\mathcal{A}_{T}$ and $\mathcal{B}_{T}$ are given by (2.1) and (2.2) (see Theorem 4.2).

We have already considered problem (1.1) in papers [7]-[11]. In [7] we proved by using potential techniques from [5] the local existence of solutions of (1.4) in Sobolev-Slobodetskiu spaces, i.e. we obtained $(u, \gamma, \eta) \in$ $W_{2}^{4,2}\left(\Omega^{T}\right) \times W_{2}^{4,2}\left(\Omega^{T}\right) \times C\left(0, T ; \Gamma^{3,3 / 2}(\Omega)\right)$ for $T \leq T_{*}$, where $T_{*}>0$ is a certain constant. We cannot apply potential theory in the present paper because this theory is singular in the case of $H^{3}(\Omega)$ regularity (with respect to the space variable $\xi$ ) considered in the paper.

Papers [8] and [9] are concerned with conservation laws and a differential inequality, respectively, used in [10]-[11] to prove the global existence theorem for problem (1.1) in the case of a special form of the internal energy per unit mass $\varepsilon=\varepsilon(\varrho, \theta)$. The main result of the present paper, i.e. Theorem 4.2, will be used in [12] to examine the global motion of the viscous compressible barotropic fluid in the general case, i.e. without assuming any conditions on the form of the pressure $p$.

In this paper we use some results of paper [6], which is concerned with the local existence of solutions of a free boundary problem for the equations of compressible barotropic viscous self-gravitating fluids.

Moreover, local existence theorems for free boundary problems for equations of compressible viscous heat-conducting and self-gravitating fluids are proved in [2] and [4].

2. Notation and auxiliary results. We use the following notation:

- $\|u\|_{s, Q}=\|u\|_{H^{s}(Q)}, s \geq 0, s$ rational, $Q=\Omega, S, S=\partial \Omega$;

- $|u|_{p, Q}=\|u\|_{L_{p}(\Omega)}, p \in[1, \infty]$;

$(0, T)$

- $\|u\|_{s, p, q, \Omega^{T}}=\|u\|_{L_{q}\left(0, T ; W_{p}^{s}(\Omega)\right)}, p, q \in[1, \infty], 0 \leq s \in \mathbb{Z}, \Omega^{T}=\Omega \times$

- $\|u\|_{s, p, q, S^{T}}=\|u\|_{L_{q}\left(0, T ; W_{p}^{s}(S)\right)}, p, q \in[1, \infty], s \geq 0, s$ rational, $S^{T}=$ $S \times(0, T)$.

Moreover, we introduce the spaces:

$$
\begin{aligned}
\mathcal{A}_{T}=\left\{u \in C\left(0, T ; H^{2}(\Omega)\right)\right. & \cap L_{2}\left(0, T ; H^{3}(\Omega)\right): \\
u_{t} & \in C\left(0, T ; H^{1}(\Omega)\right) \cap L_{2}\left(0, T ; H^{2}(\Omega)\right), \\
u_{t t} & \left.\in C\left(0, T ; L_{2}(\Omega)\right) \cap L_{2}\left(0, T ; H^{1}(\Omega)\right)\right\}
\end{aligned}
$$

and

$$
\begin{array}{r}
\mathcal{B}_{T}=\left\{u \in C\left(0, T ; H^{2}(\Omega)\right): u_{t} \in C\left(0, T ; H^{1}(\Omega)\right) \cap L_{2}\left(0, T ; H^{2}(\Omega)\right),\right. \\
\left.u_{t t} \in C\left(0, T ; L_{2}(\Omega)\right) \cap L_{2}\left(0, T ; H^{1}(\Omega)\right)\right\}
\end{array}
$$


with the norms

$$
\begin{aligned}
\|u\|_{\mathcal{A}_{T}}= & \left(\sup _{0 \leq t \leq T}\|u\|_{2, \Omega}^{2}+\|u\|_{3,2,2, \Omega^{T}}^{2}+\sup _{0 \leq t \leq T}\left\|u_{t}\right\|_{1, \Omega}^{2}\right. \\
& \left.+\left\|u_{t}\right\|_{2,2,2, \Omega^{T}}^{2}+\sup _{0 \leq t \leq T}\left\|u_{t t}\right\|_{0, \Omega}^{2}+\left\|u_{t t}\right\|_{1,2,2, \Omega^{T}}^{2}\right)^{1 / 2}
\end{aligned}
$$

and

$$
\begin{aligned}
\|u\|_{\mathcal{B}_{T}}= & \left(\sup _{0 \leq t \leq T}\|u\|_{2, \Omega}^{2}+\sup _{0 \leq t \leq T}\left\|u_{t}\right\|_{1, \Omega}^{2}+\left\|u_{t}\right\|_{2,2,2, \Omega^{T}}^{2}\right. \\
& \left.+\sup _{0 \leq t \leq T}\left\|u_{t t}\right\|_{0, \Omega}^{2}+\left\|u_{t t}\right\|_{1,2,2, \Omega^{T}}^{2}\right)^{1 / 2}
\end{aligned}
$$

Finally, define

$$
|u|_{l, k, Q}=\sum_{0 \leq i \leq l-k}\left\|\partial_{t}^{i} u\right\|_{l-i, 2,2, Q}
$$

where $l \geq k, k \in \mathbb{Z}_{+} \cup\{0\}, Q=\Omega^{t}, S^{t}$ and

$$
|u|_{l, k, \Omega}=\sum_{0 \leq i \leq l-k}\left\|\partial_{t}^{i} u\right\|_{l-i, \Omega},
$$

where $l \geq k, k \in \mathbb{Z}_{+} \cup\{0\}$.

We denote all positive constants in estimates by the same letter $c$. We also use the following lemmas.

Lemma 2.1. The following imbedding holds:

$$
W_{r}^{l}(\Omega) \subset L_{p}^{\alpha}(\Omega) \quad\left(\Omega \subset \mathbb{R}^{3}, \Omega \text { satisfies the cone condition }\right),
$$

where either

$$
\begin{aligned}
& \kappa=\frac{|\alpha|}{l}+\frac{3}{l r}-\frac{3}{l p}<1 \quad \text { and } 1 \leq r \leq p \leq \infty \text { or } \\
& \kappa=1 \quad \text { and } 1<r \leq p<\infty,
\end{aligned}
$$

and $L_{p}^{\alpha}(\Omega)$ is the space of functions $u$ such that $\left|D_{\xi}^{\alpha} u\right|_{p, \Omega}<\infty$;

$$
W_{r}^{l}(\Omega) \subset L_{q}^{\alpha}(S) \quad\left(S=\partial \Omega, \Omega \subset \mathbb{R}^{3}\right),
$$

where either

$$
\begin{aligned}
& \kappa=\frac{|\alpha|}{l}+\frac{3}{l r}-\frac{2}{l q}<1 \quad \text { and } 1 \leq r \leq q \leq \infty \quad \text { or } \\
& \kappa=1 \quad \text { and } \quad 1<r \leq q<\infty
\end{aligned}
$$

and $L_{q}^{\alpha}(S)$ is the space of functions $u$ such that $\left|D_{\xi}^{\alpha} u\right|_{q, S}<\infty$. Moreover, the following inequalities hold:

$$
\left|D_{\xi}^{\alpha} u\right|_{p, \Omega} \leq c \varepsilon^{1-\kappa}\left|D_{\xi}^{l} u\right|_{r, \Omega}+c \varepsilon^{-\kappa}|u|_{r, \Omega}
$$

where

$$
\kappa=\frac{|\alpha|}{l}+\frac{3}{l r}-\frac{3}{l p}<1, \quad 1 \leq r \leq p \leq \infty,
$$


$\varepsilon$ is a parameter and $c>0$ is a constant independent of $u$ and $\varepsilon$;

$$
\left|D_{\xi}^{\alpha} u\right|_{q, S} \leq c \varepsilon^{1-\kappa}\left|D_{\xi}^{l} u\right|_{r, \Omega}+c \varepsilon^{-\kappa}|u|_{r, \Omega}
$$

where

$$
\kappa=\frac{|\alpha|}{l}+\frac{3}{l r}-\frac{2}{l q}<1, \quad 1 \leq r \leq q \leq \infty,
$$

$\varepsilon$ is a parameter and $c>0$ is a constant independent of $u$ and $\varepsilon$.

Lemma 2.1 follows from Theorem 10.2 of [1].

Lemma 2.2. Assume that $\eta \in C\left(0, T ; H^{2}(\Omega)\right), \eta_{t} \in C\left(0, T ; H^{1}(\Omega)\right)$, $\eta_{t t} \in L_{2}\left(\Omega^{T}\right), \eta>0, \beta \in \mathcal{A}_{T}, \beta>0, c_{v} \in C^{2}\left(\mathbb{R}_{+}^{2}\right), c_{v}>0$. Then $\eta c_{v}(\eta, \beta) \in C\left(0, T ; H^{2}(\Omega)\right), \partial_{t}\left[\eta c_{v}(\eta, \beta)\right] \in C\left(0, T ; H^{1}(\Omega)\right), \partial_{t}^{2}\left[\eta c_{v}(\eta, \beta)\right] \in$ $C\left(0, T ; L_{2}(\Omega)\right), 1 /\left(\eta c_{v}(\eta, \beta)\right) \in C(\bar{\Omega} \times[0, T])$ and

$$
\begin{aligned}
& \sup _{t}\left\|\eta c_{v}(\eta, \beta)\right\|_{2, \Omega}^{2} \\
& \leq c\left\|c_{v}\right\|_{C^{2}(\bar{V})}^{2} \sup _{t}\|\eta\|_{2, \Omega}^{2} f_{1}\left(\sup _{t}\|\eta\|_{2, \Omega}^{2}, \sup _{t}\|\beta\|_{2, \Omega}^{2}\right),
\end{aligned}
$$

where $f_{1}\left(x_{1}, x_{2}\right)=1+x_{1}+x_{2}+x_{1}^{2}+x_{2}^{2}+x_{1} x_{2}$ and $c>0$ is a constant;

$$
\begin{aligned}
& \sup _{t}\left\|\partial_{t}\left[\eta c_{v}(\eta, \beta)\right]\right\|_{1, \Omega}^{2} \\
& \quad \leq c\left\|c_{v}\right\|_{C^{2}(\bar{V})}^{2} f_{2}\left(\sup _{t}\|\eta\|_{2, \Omega}^{2}, \sup _{t}\|\beta\|_{2, \Omega}^{2}, \sup _{t}\left\|\eta_{t}\right\|_{1, \Omega}^{2}, \sup _{t}\left\|\beta_{t}\right\|_{1, \Omega}^{2}\right),
\end{aligned}
$$

where $f_{2}\left(x_{1}, x_{2}, x_{3}, x_{4}\right)=x_{1}\left(x_{3}+x_{1} x_{3}+x_{2} x_{3}+x_{1} x_{4}+x_{2} x_{4}\right)+x_{3}\left(1+x_{2}+x_{4}\right)$ and $c>0$ is a constant;

$$
\begin{aligned}
\sup _{t}\left\|\partial_{t}^{2}\left[\eta c_{v}(\eta, \beta)\right]\right\|_{0, \Omega}^{2} \leq & c\left\|c_{v}\right\|_{C^{2}(\bar{V})}^{2} f_{3}\left(\sup _{t}\|\eta\|_{2, \Omega}^{2}, \sup _{t}\left\|\eta_{t}\right\|_{1, \Omega}^{2},\right. \\
& \left.\sup _{t}\left\|\beta_{t}\right\|_{1, \Omega}^{2}, \sup _{t}\left\|\eta_{t t}\right\|_{0, \Omega}^{2}, \sup _{t}\left\|\beta_{t t}\right\|_{0, \Omega}^{2}\right),
\end{aligned}
$$

where $f_{3}\left(x_{1}, x_{2}, x_{3}, x_{4}, x_{5}\right)=x_{1}\left(x_{2}^{2}+x_{2} x_{3}+x_{4}+x_{3}^{2}+x_{5}\right)+x_{2} x_{3}+x_{4}$ and $c>0$ is a constant;

$$
\begin{aligned}
& \sup _{\Omega^{T}}\left[\eta c_{v}(\eta, \beta)\right] \leq\left\|c_{v}\right\|_{C(\bar{V})} \sup _{\Omega^{T}} \eta ; \\
& \sup _{\Omega^{T}} \frac{1}{\eta c_{v}(\eta, \beta)} \leq\left\|\frac{1}{c_{v}}\right\|_{C(\bar{V})} \sup _{\Omega^{T}} \frac{1}{\eta} .
\end{aligned}
$$

In (2.5)-(2.9), $V \subset \mathbb{R}^{2}$ is a bounded domain such that $(\eta(\xi, t), \beta(\xi, t)) \in V$ for any $(\xi, t) \in \Omega^{T}$.

The proof of the above lemma is obtained using Lemma 2.1.

Now, consider the continuity equation $(1.4)_{2}$. Integrating it we have

$$
\eta(\xi, t)=\varrho_{0}(\xi) \exp \left[-\int_{0}^{t} \operatorname{div}_{u} u d t^{\prime}\right] .
$$

By direct calculations we obtain the following lemma. 
Lemma 2.3. Let $\varrho_{0} \in H^{2}(\Omega), \varrho_{0}>0, u \in L_{\infty}\left(0, T ; H^{2}(\Omega)\right) \cap L_{2}(0, T$; $\left.H^{3}(\Omega)\right), u_{t} \in L_{\infty}\left(0, T ; H^{1}(\Omega)\right) \cap L_{2}\left(0, T ; H^{2}(\Omega)\right)$. Then $\eta$ given by $(2.10)$ belongs to $\mathcal{B}_{T}$ and the following estimates hold:

$$
\begin{aligned}
\sup _{\Omega^{t}} \eta & \leq\left\|\varrho_{0}\right\|_{2, \Omega} \phi_{1}(a(u, t)), \\
\sup _{t}\|\eta\|_{2, \Omega}^{2} & \leq\left\|\varrho_{0}\right\|_{2, \Omega}^{2} \phi_{2}(a(u, t)), \\
\sup _{t}\left\|\eta_{t}\right\|_{1, \Omega}^{2} & \leq\left\|\varrho_{0}\right\|_{2, \Omega}^{2} \phi_{3}\left(a(u, t), a_{0}\left(u_{t}, t\right),\|u(0)\|_{2, \Omega}^{2}\right), \\
\sup _{t}\left\|\eta_{t t}\right\|_{0, \Omega}^{2} & \leq\left\|\varrho_{0}\right\|_{2, \Omega}^{2} \phi_{4}\left(a(u, t), a_{0}\left(u_{t}, t\right),\|u(0)\|_{2, \Omega}^{2},\left\|u_{t}(0)\right\|_{1, \Omega}^{2}\right), \\
\left\|\eta_{t}\right\|_{1,2,2, \Omega^{t}}^{2} & \leq t\left\|\varrho_{0}\right\|_{2, \Omega}^{2} \phi_{3}\left(a(u, t), a_{0}\left(u_{t}, t\right),\|u(0)\|_{2, \Omega}^{2}\right), \\
\left\|\eta_{t t}\right\|_{0, \Omega^{t}}^{2} & \leq t\left\|\varrho_{0}\right\|_{2, \Omega}^{2} \phi_{4}\left(a(u, t), a_{0}\left(u_{t}, t\right),\|u(0)\|_{2, \Omega}^{2},\left\|u_{t}(0)\right\|_{1, \Omega}^{2}\right), \\
\left\|\eta_{t}\right\|_{2,2,2, \Omega^{t}}^{2} & \leq\left\|\varrho_{0}\right\|_{2, \Omega}^{2}\|u\|_{3,2,2, \Omega^{t}}^{2} \phi_{5}\left(t, t^{a_{1}}\|u\|_{3,2,2, \Omega^{t}}^{2}\right), \\
\left\|\eta_{t t}\right\|_{1,2,2, \Omega^{t}}^{2} & \leq\left\|\varrho_{0}\right\|_{2, \Omega}^{2} \phi_{6}(a(u, t))\left[\phi_{7}\left(a(u, t), b\left(t, u, \varepsilon_{3}\right)\right)+\left\|u_{t}\right\|_{2,2,2, \Omega^{t}}^{2}\right],
\end{aligned}
$$

where $t \leq T, \phi_{i}(i=1, \ldots, 7)$ are positive increasing continuous functions of their arguments, $a(u, t)=t \int_{0}^{t}\|u\|_{3, \Omega}^{2} d t^{\prime}, a_{0}\left(u_{t}, t\right)=t \int_{0}^{t}\left\|u_{t}\right\|_{2, \Omega}^{2} d t^{\prime}, b$ is given by (3.46) and $a_{1}>0$ is a constant. Moreover, $1 / \eta \in \mathcal{B}_{T}$ and

$$
\sup _{\Omega^{t}} \frac{1}{\eta}+\left\|\frac{1}{\eta}\right\|_{\mathcal{B}_{t}}^{2} \leq \phi_{8}\left(\|u\|_{3,2,2, \Omega^{t}}^{2}, \sup _{t}\|u\|_{2, \Omega}^{2},\left\|u_{t}\right\|_{2,2,2, \Omega^{t}}^{2}, \sup _{t}\left\|u_{t}\right\|_{2, \Omega}^{2}\right),
$$

where $t \leq T$ and $\phi_{8}$ is a positive increasing continuous function of its arguments.

3. Existence of solutions of auxiliary problems. In order to prove the local-in-time solvability of problem (1.4) we have to consider a few auxiliary problems. First, we consider the problem

$$
\begin{array}{ll}
\eta u_{t}-\operatorname{div} \mathbb{D}(u)=F & \text { in } \Omega^{T}, \\
\mathbb{D}(u) \cdot \bar{n}_{0}=G & \text { on } S^{T}, \\
\left.u\right|_{t=0}=v_{0} & \text { in } \Omega,
\end{array}
$$

where $\mathbb{D}(u)$ is defined by (1.2) and $\eta$ is a given function. Moreover,

$$
0<\varrho_{*} \leq \eta \leq \varrho^{*}<\infty,
$$

where $\varrho_{*}$ and $\varrho^{*}$ are constants.

DeFinition 3.1. By a weak solution of problem (3.1) we mean a function $u \in C\left(0, T ; L_{2}(\Omega)\right) \cap L_{2}\left(0, T ; H^{1}(\Omega)\right)$ with $u_{t} \in L_{2}\left(\Omega^{T}\right)$ which satisfies the integral identity

$$
\int_{\Omega}\left[\eta u_{t} \phi+\frac{\mu}{2} \mathbb{S}(u) \mathbb{S}(\phi)+(\nu-\mu) \operatorname{div} u \operatorname{div} \phi-F \phi\right] d \xi-\int_{S} G \phi d \xi_{s}=0
$$


for all $\phi \in H^{1}(\Omega)$ and the initial condition

$$
\left.u\right|_{t=0}=v_{0} ;
$$

here $f \phi=\sum_{i=1}^{3} f_{i} \phi_{i}, f=u_{t}, F, G, \mathbb{S}(u)=\left\{u_{i \xi_{j}}+u_{j \xi_{i}}\right\}_{i, j=1,2,3}$ and

$$
\mathbb{S}(u) \mathbb{S}(\phi)=\sum_{i, j=1}^{3}\left(u_{i \xi_{j}}+u_{j \xi_{i}}\right)\left(\phi_{i \xi_{j}}+\phi_{j \xi_{i}}\right) .
$$

In order to prove the existence of a weak solution to problem (3.1) we shall apply a Galerkin procedure. Choose a sequence of functions $\phi_{1}, \phi_{2}, \ldots$ such that: $\phi_{i} \in H^{1}(\Omega)$ for all $i ; \phi_{1}, \ldots, \phi_{n}$ are linearly independent for each $n$; the set of all linear combinations of the functions $\phi_{i}$ is dense in $H^{1}(\Omega)$. For any $n$ we define an approximate solution of problem (3.1) by

$$
\begin{aligned}
& u_{n}=\sum_{i=1}^{n} c_{i n}(t) \phi_{i}(\xi), \\
& \int_{\Omega}\left[\eta u_{n t} \phi_{i}+\frac{\mu}{2} \mathbb{S}\left(u_{n}\right) \mathbb{S}\left(\phi_{i}\right)+(\nu-\mu) \operatorname{div} u_{n} \operatorname{div} \phi_{i}-F \phi_{i}\right] d \xi \\
& -\int_{S} G \phi_{i} d \xi_{s}=0, \\
& u_{n}(0)=u_{n 0},
\end{aligned}
$$

where $u_{n 0} \rightarrow v_{0}$ in $H^{1}(\Omega), u_{n t}(0) \rightarrow u_{t}(0)$ in $H^{1}(\Omega), u_{n t t}(0) \rightarrow u_{t t}(0)$ in $L_{2}(\Omega)$ and $\left\|u_{n 0}\right\|_{1, \Omega} \leq c\left\|v_{0}\right\|_{1, \Omega},\left\|u_{n t}(0)\right\|_{1, \Omega} \leq c\left\|u_{t}(0)\right\|_{1, \Omega},\left|u_{n t t}(0)\right|_{2, \Omega} \leq$ $c\left|u_{t t}(0)\right|_{2, \Omega} ; u_{t}(0)$ and $u_{t t}(0)$ are calculated from (3.1); $c>0$ is a constant.

Lemma 3.2. Let assumption (3.2) be satisfied. Let $\eta \in C(\bar{\Omega} \times[0, T]), \eta_{t} \in$ $L_{2}\left(0, T ; H^{1}(\Omega)\right), \eta_{t t} \in L_{2}\left(\Omega^{T}\right), F \in H^{2}\left(0, T ; L_{2}(\Omega)\right), G \in H^{2}\left(0, T ; L_{2}(S)\right)$, $v_{0} \in H^{1}(\Omega), u_{t}(0) \in H^{1}(\Omega), u_{t t}(0) \in L_{2}(\Omega)$. Then there exists a unique weak solution of problem (3.1) such that $u \in L_{\infty}\left(0, T ; H^{1}(\Omega)\right), u_{t} \in L_{\infty}(0, T$; $\left.H^{1}(\Omega)\right), u_{t t} \in L_{\infty}\left(0, T ; L_{2}(\Omega)\right) \cap L_{2}\left(0, T ; H^{1}(\Omega)\right)$ and the following estimate is satisfied:

$$
\begin{aligned}
&\|u\|_{1, \Omega}^{2}+\left\|u_{t}\right\|_{1, \Omega}^{2}+\left\|u_{t t}\right\|_{0, \Omega}^{2}+\|u\|_{1,2,2, \Omega^{t}}^{2}+\left\|u_{t}\right\|_{1,2,2, \Omega^{t}}^{2}+\left\|u_{t t}\right\|_{1,2,2, \Omega^{t}}^{2} \\
& \leq \Psi_{1}\left(1 / \varrho_{*}, \varrho^{*}, t,\left\|\eta_{t}\right\|_{1,2,2, \Omega^{t}}^{2},\left\|\eta_{t t}\right\|_{0, \Omega^{t}}^{2}\right)\left[\|F\|_{0, \Omega^{t}}^{2}+\left\|F_{t}\right\|_{0, \Omega^{t}}^{2}\right. \\
&+\varepsilon_{1}\left\|F_{t t}\right\|_{0, \Omega^{t}}^{2}+\|G\|_{0, S^{t}}^{2}+\left\|G_{t}\right\|_{0, S^{t}}^{2} \\
&\left.+\varepsilon_{1}\left\|G_{t t}\right\|_{0, S^{t}}^{2}+\left\|v_{0}\right\|_{1, \Omega}^{2}+\left\|u_{t}(0)\right\|_{1, \Omega}^{2}+\left\|u_{t t}(0)\right\|_{0, \Omega}^{2}\right],
\end{aligned}
$$

where $t \leq T, \Psi_{1}$ is a positive increasing continuous function of its arguments and $\varepsilon_{1} \in(0,1)$ is a sufficiently small constant. 
Proof. First, multiply (3.4) by $c_{i n}$ and sum up over $i$ from 1 to $n$. Using the Korn inequality and Lemma 2.1 we get

$$
\begin{aligned}
\frac{d}{d t} \int_{\Omega} \eta u_{n}^{2} d \xi+c\left\|u_{n}\right\|_{1, \Omega}^{2} \leq & \frac{c}{\varrho_{*}}\left(1+\left\|\eta_{t}\right\|_{1, \Omega}^{2}\right) \int_{\Omega} \eta u_{n}^{2} d \xi \\
& +c\left(\|F\|_{0, \Omega}^{2}+\|G\|_{0, S}^{2}\right)+\varepsilon\left\|u_{n}\right\|_{1, \Omega}^{2},
\end{aligned}
$$

where we have also used the fact that

$$
\frac{\mu}{2} \int_{\Omega}\left|\mathbb{S}\left(u_{n}\right)\right|^{2} d \xi+(\nu-\mu)\left\|\operatorname{div} u_{n}\right\|_{0, \Omega}^{2} \geq c \int_{\Omega}\left|\mathbb{S}\left(u_{n}\right)\right|^{2} d \xi .
$$

Hence, integrating with respect to time, taking $\varepsilon>0$ sufficiently small and using the Gronwall inequality we have

$$
\begin{aligned}
\int_{\Omega} \eta u_{n}^{2} d \xi+\left\|u_{n}\right\|_{1,2,2, \Omega^{t}}^{2} \leq & \Psi_{2}\left(1 / \varrho_{*}, \varrho^{*}, t,\left\|\eta_{t}\right\|_{1,2,2, \Omega^{t}}^{2}\right) \\
& \times\left(\int_{\Omega} \eta(0) v_{0}^{2} d \xi+\|F\|_{0, \Omega^{t}}^{2}+\|G\|_{0, S^{t}}^{2}\right),
\end{aligned}
$$

where $\Psi_{2}$ is a positive increasing continuous function.

Next, multiplying (3.4) by $\dot{c}_{i n}$ and summing up over $i$ we obtain

$$
\begin{aligned}
\int_{\Omega}\left\{\eta u_{n t}^{2}+\frac{1}{2} \frac{d}{d t}\left[\frac{\mu}{2}\left|\mathbb{S}\left(u_{n}\right)\right|^{2}+(\nu-\mu)\left(\operatorname{div} u_{n}\right)^{2}\right]\right. & \left.-F u_{n t}\right\} d \xi \\
& -\int_{S} G u_{n t} d \xi_{s}=0 .
\end{aligned}
$$

Integrating (3.7) with respect to $t$ and using the Korn inequality yields

$$
\begin{aligned}
\int_{\Omega^{t}} \eta u_{n t}^{2} d \xi d t^{\prime}+\left\|u_{n}\right\|_{1, \Omega}^{2} \leq & \varepsilon\left\|u_{n t}\right\|_{1,2,2, \Omega^{t}}^{2}+c\left\|u_{n}\right\|_{0, \Omega}^{2} \\
& +c\left(\|F\|_{0, \Omega^{t}}^{2}+\|G\|_{0, S^{t}}^{2}+\left\|v_{0}\right\|_{1, \Omega}^{2}\right) .
\end{aligned}
$$

Differentiating (3.4) with respect to $t$, multiplying by $\dot{c}_{i n}$ and summing up over $i$ from 1 to $n$ we get

$$
\begin{gathered}
\frac{d}{d t} \int_{\Omega} \eta u_{n t}^{2} d \xi+c\left\|u_{n t}\right\|_{1, \Omega}^{2} \leq \\
\varepsilon\left\|u_{n t}\right\|_{1, \Omega}^{2}+\frac{c}{\varrho_{*}}\left(1+\left\|\eta_{t}\right\|_{1, \Omega}^{2}\right) \int_{\Omega} \eta u_{n t}^{2} d \xi \\
+c\left(\left\|F_{t}\right\|_{0, \Omega}^{2}+\left\|G_{t}\right\|_{0, S}^{2}\right) .
\end{gathered}
$$

Integrating (3.9) with respect to time gives

$$
\begin{aligned}
& \int_{\Omega} \eta u_{n t}^{2} d \xi+\left\|u_{n t}\right\|_{1,2,2, \Omega^{t}}^{2} \\
\leq & \Psi_{2}\left(1 / \varrho_{*}, \varrho^{*}, t,\left\|\eta_{t}\right\|_{1,2,2, \Omega^{t}}^{2}\right)\left(\int_{\Omega} \eta(0) u_{t}^{2}(0) d \xi+\left\|F_{t}\right\|_{0, \Omega^{t}}^{2}+\left\|G_{t}\right\|_{0, S^{t}}^{2}\right) .
\end{aligned}
$$


Next, differentiate (3.4) with respect to $t$, multiply the result by $\ddot{c}_{i n}$ and sum up over $i$ from 1 to $n$. We obtain

$$
\begin{aligned}
\int_{\Omega} \eta u_{n t t}^{2} d \xi+ & \frac{1}{2} \frac{d}{d t} \int_{\Omega}\left[\frac{\mu}{2}\left|\mathbb{S}\left(u_{n} t\right)\right|^{2}+(\nu-\mu)\left(\operatorname{div} u_{n t}\right)^{2}\right] d \xi \\
& \leq \varepsilon\left\|u_{n t t}\right\|_{1, \Omega}^{2}+c\left(\left\|\eta_{t}\right\|_{1, \Omega}^{2}\left\|u_{n t}\right\|_{0, \Omega}^{2}+\left\|F_{t}\right\|_{0, \Omega}^{2}+\left\|G_{t}\right\|_{0, S}^{2}\right) .
\end{aligned}
$$

Hence

$$
\begin{aligned}
\int_{\Omega^{t}} \eta u_{n t t}^{2} d \xi d t^{\prime} & +\left\|u_{n t}\right\|_{1, \Omega}^{2} \\
\leq & \varepsilon\left\|u_{n t t}\right\|_{1,2,2, \Omega^{t}}^{2}+c\left(\left\|\eta_{t}\right\|_{1,2,2, \Omega^{t}}^{2} \sup _{t}\left\|u_{n t}\right\|_{0, \Omega}^{2}\right. \\
& \left.+\left\|F_{t}\right\|_{0, \Omega^{t}}^{2}+\left\|G_{t}\right\|_{0, S^{t}}^{2}+\left\|u_{n t}\right\|_{0, \Omega^{t}}^{2}+\left\|u_{n t \xi}(0)\right\|_{0, \Omega}^{2}\right) .
\end{aligned}
$$

Finally, differentiating twice $(3.4)$ with respect to $t$, multiplying by $\ddot{c}_{i n}$ and summing up over $i$ we have

$$
\begin{aligned}
\frac{d}{d t} \int_{\Omega} \eta u_{n t t}^{2} d \xi+c\left\|u_{n t t}\right\|_{1, \Omega}^{2} \leq & \frac{c}{\varrho_{*}}\left(\left\|\eta_{t}\right\|_{1, \Omega}^{2}+1\right) \int_{\Omega} \eta u_{n t t}^{2} d \xi \\
& +c\left(\left\|\eta_{t t}\right\|_{0, \Omega}^{2}\left\|u_{n t}\right\|_{1, \Omega}^{2}+\varepsilon_{1}\left\|F_{t t}\right\|_{0, \Omega}^{2}\right. \\
& \left.+\varepsilon_{1}\left\|G_{t t}\right\|_{0, S}^{2}\right) .
\end{aligned}
$$

Therefore, integrating (3.12) with respect to time yields

$$
\begin{aligned}
& \quad \int_{\Omega} \eta u_{n t t}^{2} d \xi+\left\|u_{n t t}\right\|_{1,2,2, \Omega^{t}}^{2} \\
& \leq \Psi_{2}\left(1 / \varrho_{*}, \varrho^{*}, t,\left\|\eta_{t}\right\|_{1,2,2, \Omega^{t}}^{2}\right)\left(\int_{\Omega} \eta(0) u_{t t}^{2}(0) d \xi+\varepsilon_{1}\left\|F_{t t}\right\|_{0, \Omega^{t}}^{2}+\varepsilon_{1}\left\|G_{t t}\right\|_{0, S^{t}}^{2}\right. \\
& \left.\quad+\sup _{t}\left\|u_{n t}\right\|_{1, \Omega}^{2}\left\|\eta_{t t}\right\|_{0, \Omega^{t}}^{2}\right) .
\end{aligned}
$$

Using inequality (3.11) in (3.13) and assuming that $\varepsilon \Psi_{2}\left\|\eta_{t t}\right\|_{0, \Omega^{t}}^{2}$ is sufficiently small we obtain

$$
\begin{aligned}
& \int_{\Omega} \eta u_{n t t}^{2} d \xi+\left\|u_{n t t}\right\|_{1,2,2, \Omega^{t}}^{2} \\
\leq & \Psi_{3}\left(1 / \varrho_{*}, \varrho^{*}, t,\left\|\eta_{t}\right\|_{1,2,2, \Omega^{t}}^{2},\left\|\eta_{t t}\right\|_{0, \Omega^{t}}^{2}\right)\left(\int_{\Omega} \eta(0) u_{t t}^{2}(0) d \xi+\left\|u_{t \xi}(0)\right\|_{0, \Omega}^{2}\right. \\
& +\varepsilon_{1}\left\|F_{t t}\right\|_{0, \Omega^{t}}^{2}+\varepsilon_{1}\left\|G_{t t}\right\|_{0, S^{t}}^{2}+\left\|F_{t}\right\|_{0, \Omega^{t}}^{2}+\left\|G_{t}\right\|_{0, S^{t}}^{2} \\
& \left.+\left\|u_{n t}\right\|_{0, \Omega^{t}}^{2}+\sup _{t}\left\|u_{n t}\right\|_{0, \Omega}^{2}\right),
\end{aligned}
$$

where $\Psi_{3}$ is a positive increasing continuous function of its arguments. 
Now, taking into account inequalities (3.6), (3.8), (3.10), (3.11) and (3.14) we get the estimate

$$
\begin{aligned}
\left\|u_{n}\right\|_{1, \Omega}^{2}+\left\|u_{n t}\right\|_{1, \Omega}^{2} & +\left\|u_{n t t}\right\|_{0, \Omega}^{2}+\left\|u_{n}\right\|_{1,2,2, \Omega^{t}}^{2}+\left\|u_{n t}\right\|_{1,2,2, \Omega^{t}}^{2}+\left\|u_{n t t}\right\|_{1,2,2, \Omega^{t}}^{2} \\
\leq & \Psi_{1}\left(1 / \varrho_{*}, \varrho^{*}, t,\left\|\eta_{t}\right\|_{1,2,2, \Omega^{t}}^{2},\left\|\eta_{t t}\right\|_{0, \Omega^{t}}^{2}\right]\left[\|F\|_{0, \Omega^{t}}^{2}+\left\|F_{t}\right\|_{0, \Omega^{t}}^{2}\right. \\
& +\varepsilon_{1}\left\|F_{t t}\right\|_{0, \Omega^{t}}^{2}+\|G\|_{0, S^{t}}^{2}+\left\|G_{t}\right\|_{0, S^{t}}^{2} \\
& +\varepsilon_{1}\left\|G_{t t}\right\|_{0, S^{t}}^{2}+\int_{\Omega} \eta(0) v_{0}^{2} d \xi+\int_{\Omega} \eta(0) u_{t}^{2}(0) d \xi \\
& \left.+\int_{\Omega} \eta(0) u_{t t}^{2}(0) d \xi+\left\|v_{0}\right\|_{1, \Omega}^{2}+\left\|u_{t \xi}(0)\right\|_{0, \Omega}^{2}\right] .
\end{aligned}
$$

Choosing a subsequence and letting $n \rightarrow \infty$ we obtain the existence of a solution of (3.1) and estimate (3.5). Uniqueness follows from (3.5). This concludes the proof.

Lemma 3.3. Let assumption (3.2) be satisfied. Let $\eta \in C\left(0, T ; H^{2}(\Omega)\right)$, $\eta_{t} \in C\left(0, T ; H^{1}(\Omega)\right), 1 / \eta \in C\left(0, T ; H^{2}(\Omega)\right),(1 / \eta)_{t} \in C\left(0, T ; H^{1}(\Omega)\right), \eta_{t t} \in$ $L_{2}\left(\Omega^{T}\right), F \in H^{2}\left(0, T ; L_{2}(\Omega)\right) \cap L_{2}\left(0, T ; H^{1}(\Omega)\right), G \in L_{2}\left(0, T ; H^{3 / 2}(S)\right)$, $\left.G_{t} \in L_{2}\left(0, T ; H^{1 / 2}(S)\right), G_{t t} \in L_{2}\left(S^{T}\right)\right), S \in H^{5 / 2}, v_{0} \in H^{2}(\Omega), u_{t}(0) \in$ $H^{1}(\Omega), u_{t t}(0) \in L_{2}(\Omega)$ (where $u_{t}(0)$ and $u_{t t}(0)$ are calculated from (3.1)). Moreover, let the compatibility condition be satisfied:

$$
\mathbb{D}\left(v_{0}\right) \cdot \bar{n}_{0}=G(0) \quad \text { on } S .
$$

Then the solution $u$ of problem (3.1) belongs to $\mathcal{A}_{T}$ and for $t \leq T$ the following estimate holds:

$$
\begin{aligned}
\|u\|_{\mathcal{A}_{t}}^{2} \leq & \Psi_{4}\left(1 / \varrho_{*}, \varrho^{*}, t, h\left(t, \eta, \varepsilon_{2}\right)\right)\left[\|F\|_{1,2,2, \Omega^{t}}^{2}+\left\|F_{t}\right\|_{0, \Omega^{t}}^{2}\right. \\
& +\varepsilon_{1}\left\|F_{t t}\right\|_{0, \Omega^{t}}^{2}+\sup _{t}\|F\|_{0, \Omega}^{2}+\|G\|_{3 / 2,2,2, S^{t}}^{2} \\
& \left.+\left\|G_{t}\right\|_{1 / 2,2,2, S^{t}}^{2}+\varepsilon_{1}\left\|G_{t t}\right\|_{0, S^{t}}^{2}+|u(0)|_{2,0, \Omega}^{2}\right],
\end{aligned}
$$

where $\varepsilon_{i} \in(0,1)(i=1,2)$ are sufficiently small constants, $\Psi_{4}$ is a positive increasing continuous function of its arguments depending also on $\|\Phi\|_{3, \Omega}^{2}$ (where $\Phi$ is a transformation which straightens locally the boundary of $\Omega$ ) and

$$
\begin{aligned}
h\left(t, \eta, \varepsilon_{2}\right)= & \left\|\eta_{t}\right\|_{1,2,2, \Omega^{t}}^{2}+\left\|\eta_{t t}\right\|_{0, \Omega^{t}}^{2}+\varepsilon_{2} \sup _{t}\|\eta\|_{2, \Omega}^{2} \\
& +c\left(\varepsilon_{2}\right) \sup _{t}\|\eta\|_{0, \Omega}^{2}+\varepsilon_{2} \sup _{t}\left\|\eta_{t}\right\|_{1, \Omega}^{2}+c\left(\varepsilon_{2}\right) \sup _{t}\left\|\eta_{t}\right\|_{0, \Omega}^{2} .
\end{aligned}
$$

Proof. In [6] it is proved that the solution $u$ of problem (3.1) belongs to $L_{2}\left(0, T ; H^{3}(\Omega)\right)$, so in view of Lemma 3.2 it suffices to prove that $u \in C\left(0, T ; H^{2}(\Omega)\right)$ with $u_{t} \in C\left(0, T ; H^{1}(\Omega)\right) \cap L_{2}\left(0, T ; H^{2}(\Omega)\right), u_{t t} \in$ $C\left(0, T ; L_{2}(\Omega)\right)$ and that the estimate (3.15) holds. In order to do it con- 
sider as in [6] a covering $\left\{\Omega_{j}\right\}_{j=1}^{n}$ of $\Omega$ and associate with this covering a partition of unity $\left\{\zeta_{j}\right\}_{j=1}^{n}$, i.e. $\sum_{j=1}^{n} \zeta_{j}=1, \operatorname{supp} \zeta_{j} \subset \Omega_{j}, \zeta_{j} \in C_{0}^{\infty}\left(\mathbb{R}^{3}\right)$. Denote by $\widetilde{\Omega}$ an arbitrary set of the covering $\left\{\Omega_{j}\right\}$ such that $\widetilde{\Omega} \cap S=\emptyset$. Denote by $\zeta$ a function of the partition of unity $\left\{\zeta_{j}\right\}$ such that $\operatorname{supp} \zeta \subset \widetilde{\Omega}$. Since the identity (3.3) is satisfied for any test function $\phi$ it is also fulfilled for $\zeta \phi$. Then we have

$$
\begin{aligned}
\int_{\widetilde{\Omega}}\left[\eta u_{t} \zeta \phi+\frac{\mu}{2} \mathbb{S}(u) \mathbb{S}(\zeta \phi)+(\nu-\mu) \operatorname{div} u\right. & \operatorname{div}(\zeta \phi)-F \zeta \phi] d \xi \\
& -\int_{S \cap \widetilde{\Omega}=\widetilde{S}} G \zeta \phi d \xi_{s}=0 .
\end{aligned}
$$

Now, apply the transformation $\Phi: \widetilde{\Omega} \rightarrow \widehat{\Omega}$ which straightens locally the boundary of $\Omega$. Then (3.17) takes the form

$$
\begin{aligned}
& \int_{\widehat{\Omega}}\left\{\widehat{\eta} \widetilde{u}_{t} \widehat{\phi}+\frac{\mu}{2}\left[\widehat{\mathbb{S}}(\widetilde{u}) \widehat{\mathbb{S}}(\widehat{\phi})+\widehat{\mathbb{S}}(\widetilde{u}) \widehat{\mathbb{D}}_{1}(\widehat{\zeta}, \widehat{\phi})-\widehat{\mathbb{D}}_{1}(\widehat{u}, \widehat{\zeta}) \widehat{\mathbb{S}}(\widehat{\phi})\right]\right\} J d z \\
& +\int_{\widehat{\Omega}}\{(\nu-\mu)[\widehat{\operatorname{div}} \widetilde{u} \widehat{\operatorname{div}} \widehat{\phi}+\widehat{\operatorname{div}} \widehat{u} \widehat{\phi} \cdot \widehat{\nabla} \widehat{\zeta}-\widehat{u} \cdot \widehat{\nabla} \widehat{\zeta} \widehat{\operatorname{div}} \widehat{\phi}]-\widehat{F} \widehat{\zeta} \widehat{\phi}\} J d z \\
& -\int_{\widehat{S}} \widehat{G} \widehat{\zeta} \widehat{\phi} \sqrt{g} d z_{s}=0,
\end{aligned}
$$

where $\widetilde{\Omega} \ni \xi \rightarrow \Phi(\xi)=z \in \widehat{\Omega}, \widehat{u}=u \circ \Phi^{-1}, \widetilde{u}=\widehat{u} \widehat{\zeta}, J$ is the Jacobian of the transformation $\xi=\Phi^{-1}(z)=\left(z_{1}, z_{2}, z_{3}+\widetilde{\psi}(z)\right), \widetilde{\psi}$ is an extension to $\widehat{\Omega}$ of a function $\psi$ such that $\widetilde{S}$ is described by $\xi_{3}=\psi\left(\xi_{1}, \xi_{2}\right), g=1+\psi_{z_{1}}^{2}+\psi_{z_{2}}^{2}$, $\nabla_{\xi}$ in $\mathbb{S}, \widehat{\mathbb{D}}_{1}$, etc. is replaced by $\widehat{\nabla}=\left.\nabla_{\xi} \Phi(\xi)\right|_{\xi=\Phi^{-1}(z)} \cdot \nabla_{z}$, and $\widehat{\mathbb{D}_{1}}(\widehat{\zeta}, \widehat{w})=$ $\left\{\widehat{w}_{i} \widehat{\nabla}_{j} \widehat{\zeta}+\widehat{w}_{j} \widehat{\nabla}_{i} \widehat{\zeta}\right\}_{i, j=1,2,3}\left(w=\phi, u ; \widehat{\nabla}=(\widehat{\nabla})_{i=1,2,3}\right)$

Moreover, we need that $\widehat{\Omega}=\left\{z \in \mathbb{R}^{3}:\left|z_{i}\right|<d, i=1,2,0<z_{3}<d\right\}$, $\widehat{S}=\Phi(\widetilde{S})=\left\{z \in \mathbb{R}^{3}:\left|z_{i}\right|<d, i=1,2, z_{3}=0\right\}$. Since the first integral in (3.18) vanishes on $\partial \widehat{\Omega} \backslash \widehat{S}$ it can be extended by zero onto $\mathbb{R}_{+}^{3}=\left\{z \in \mathbb{R}^{3}\right.$ : $\left.z_{3}>0\right\}$.

Assume in (3.18) (as in [6]) $\widehat{\phi}=\delta_{n}^{-1} \delta_{n} \widetilde{u}$, where

$$
\begin{gathered}
\delta_{h} u(z)=\frac{1}{h}\left[u\left(z^{\prime}+h, z_{3}\right)-u(z)\right], \quad \delta_{h}^{-1} u(z)=\frac{1}{h}\left[u\left(z^{\prime}-h, z_{3}\right)-u(z)\right], \\
z^{\prime}=\left(z_{1}, z_{2}\right) .
\end{gathered}
$$

Then the first term in (3.18) can be rewritten as (see (A.7) in [6])

$$
\begin{aligned}
\int_{\widehat{\Omega}} \widehat{\eta} \widetilde{u}_{t} \widehat{\phi} J d z= & -\int_{\widehat{\Omega}} \delta_{h} \widehat{\eta} \widetilde{u}_{t} \delta_{h} \widetilde{u} J d z-\int_{\widehat{\Omega}} \widehat{\eta} \widetilde{u}_{t} \delta_{h} \widetilde{u} \delta_{h} J d z \\
& -\frac{1}{2} \frac{d}{d t} \int_{\widehat{\Omega}} \widehat{\eta}\left|\delta_{h} \widetilde{u}\right|^{2} J d z+\frac{1}{2} \int_{\widehat{\Omega}} \widehat{\eta}_{t}\left|\delta_{h} \widetilde{u}\right|^{2} J d z
\end{aligned}
$$


and by Lemma 2.1 the first two terms in (3.19) are bounded by

$$
\varepsilon\left\|\delta_{h} \widetilde{u}\right\|_{0, \widehat{\Omega}}^{2}+c(\varepsilon)\left(\varepsilon_{2}\|\widehat{\eta}\|_{2, \widehat{\Omega}}^{2}+c\left(\varepsilon_{2}\right)\|\widehat{\eta}\|_{0, \widehat{\Omega}}^{2}\right)\left\|\widetilde{u}_{t}\right\|_{1, \widehat{\Omega}}^{2},
$$

where $\varepsilon \in(0,1)$ and $\varepsilon_{2} \in(0,1)$. Hence in the same way as in [6] we obtain (cf. inequality (A.8) of $[6]$ ) the estimate

$$
\begin{aligned}
\frac{1}{2} \frac{d}{d t} \int_{\widehat{\Omega}} \widehat{\eta}\left|\delta_{h} \widetilde{u}\right|^{2} J d z+\frac{\mu}{2}\left\|\delta_{h} \widetilde{u}\right\|_{1, \widehat{\Omega}}^{2} \\
\leq c\left(\varepsilon_{2}\|\widehat{\eta}\|_{2, \widehat{\Omega}}^{2}+c\left(\varepsilon_{2}\right)\|\widehat{\eta}\|_{0, \widehat{\Omega}}^{2}\right)\left\|\widetilde{u}_{t}\right\|_{1, \widehat{\Omega}}^{2}+c\left\|\widehat{\eta}_{t}\right\|_{1, \widehat{\Omega}}^{2}\left\|\delta_{h} \widetilde{u}\right\|_{0, \widehat{\Omega}}^{2} \\
\quad+\varepsilon\left\|\delta_{h} \widehat{u}\right\|_{1, \widehat{\Omega}}^{2}+c\|\widehat{u}\|_{1, \widehat{\Omega}}^{2}+c\left(\|\widetilde{F}\|_{0, \widehat{\Omega}}^{2}+\|\widetilde{G}\|_{1,2, \widehat{S}}^{2}\right) .
\end{aligned}
$$

Integrating (3.20) with respect to time, going back to the old variables, summing over all neighborhoods of the partition of unity, using the fact that $\varepsilon$ is sufficiently small and letting $h$ tend to 0 we get

$$
\begin{aligned}
& \int_{\Omega} \eta u_{\tau}^{2} d \xi+\mu\left\|u_{\tau}\right\|_{1,2,2, \Omega^{t}}^{2} \\
\leq & \int_{\Omega} \eta(0) v_{0 \tau}^{2} d \xi+\left(\varepsilon_{2} \sup _{t}\|\eta\|_{2, \Omega}^{2}+c\left(\varepsilon_{2}\right) \sup _{t}\|\eta\|_{0, \Omega}^{2}\right)\left\|u_{t}\right\|_{1,2,2, \Omega^{t}}^{2} \\
& +c\left\|\eta_{t}\right\|_{1,2,2, \Omega^{t}}^{2} \sup _{t}\|u\|_{1, \Omega}^{2}+c\left(\|u\|_{1,2,2, \Omega^{t}}^{2}+\|F\|_{0, \Omega^{t}}^{2}+\|G\|_{1 / 2,2,2, S^{t}}\right),
\end{aligned}
$$

where $u_{\tau}$ denotes the tangent derivatives to the boundary and the constant $c$ depends on $\|\Phi\|_{3, \Omega}^{2}$.

To calculate the normal derivatives we use the equation

$$
-\operatorname{div} \mathbb{D}(u)=F-\eta u_{t} .
$$

Hence we have

$$
\left\|u_{n}\right\|_{1, \Omega}^{2} \leq c\left(\|F\|_{0, \Omega}^{2}+\left\|u_{\tau}\right\|_{1, \Omega}^{2}\right)+c\left(\varepsilon_{2}\|\eta\|_{1, \Omega}^{2} c\left(\varepsilon_{2}\right)\|\eta\|_{0, \Omega}^{2}\right)\left\|u_{t}\right\|_{1, \Omega}^{2} .
$$

Now, inequalities (3.21) and (3.22) imply

$$
\begin{aligned}
& \int_{\Omega} \eta u_{\tau}^{2} d \xi+\|u\|_{2,2,2, \Omega^{t}}^{2} \\
\leq & \int_{\Omega} \eta(0) v_{0 \tau}^{2} d \xi+\left(\varepsilon_{2} \sup _{t}\|\eta\|_{2, \Omega}^{2}+c\left(\varepsilon_{2}\right) \sup _{t}\|\eta\|_{0, \Omega}^{2}\right)\left\|u_{t}\right\|_{1,2,2, \Omega^{t}}^{2} \\
& +c\left\|\eta_{t}\right\|_{1,2,2, \Omega^{t}}^{2} \sup _{t}\|u\|_{1, \Omega}^{2}+c\left(\|u\|_{1,2,2, \Omega^{t}}^{2}+\|F\|_{0, \Omega^{t}}^{2}+\|G\|_{1 / 2,2,2, S^{t}}^{2}\right),
\end{aligned}
$$

where $t \leq T$ and the r.h.s. of (3.23) is bounded in terms of the estimates for the weak solution (see Lemma 3.2).

Now, we obtain estimates for $\sup _{t}\|u\|_{2, \Omega}^{2}$ and $\left\|u_{t}\right\|_{2,2,2, \Omega^{t}}^{2}$. To do this we put $\widehat{\phi}=\delta_{h}^{-1} \delta_{h} \widetilde{u}_{t}$ in (3.18). 
Using the Hölder and Young inequalities and Lemma 2.1 we obtain

$$
\begin{array}{rl}
\int_{\widehat{\Omega}} \widehat{\eta}\left|\delta_{h} \widetilde{u}_{t}\right|^{2} J & d z+\frac{1}{2} \frac{d}{d t} \int_{\widehat{\Omega}}\left[\frac{\mu}{2}\left|\widehat{\mathbb{S}}\left(\delta_{n} \widetilde{u}\right)\right|^{2}+(\nu-\mu)\left(\widehat{\operatorname{div}} \delta_{n} \widetilde{u}\right)^{2}\right] J d z \\
\leq & \varepsilon\left\|\delta_{h} \widetilde{u}_{t}\right\|_{1, \widehat{\Omega}}^{2}+c\left[\left\|\widetilde{u}_{t}\right\|_{1, \widehat{\Omega}}^{2}\left(\varepsilon_{2}\left\|\delta_{h} \widehat{\eta}\right\|_{1, \widehat{\Omega}}^{2}+c\left(\varepsilon_{2}\right)\left\|\delta_{h} \widehat{\eta}\right\|_{0, \widehat{\Omega}}^{2}\right)\right. \\
& +\left\|\widetilde{u}_{t}\right\|_{1, \widehat{\Omega}}^{2}\left(\varepsilon_{2}\|\widehat{\eta}\|_{2, \widehat{\Omega}}^{2}+c\left(\varepsilon_{2}\right)\|\widehat{\eta}\|_{0, \widehat{\Omega}}^{2}\right)\left\|\delta_{h} J\right\|_{1, \widehat{\Omega}}^{2}+\|\widehat{u}\|_{1, \widehat{\Omega}}^{2} \\
& \left.+\|\widetilde{u}\|_{2, \widehat{\Omega}}^{2}\left\|\delta_{h} J\right\|_{1, \widehat{\Omega}}^{2}+\|\widetilde{F}\|_{0, \widehat{\Omega}}^{2}+\|\widetilde{G}\|_{1 / 2, \widehat{S}}^{2}\right] .
\end{array}
$$

Integrating (3.24) with respect to time and using the Korn inequality yields

$$
\begin{aligned}
& \int_{\widehat{\Omega}^{t}} \widehat{\eta}\left|\delta_{h} \widetilde{u}_{t}\right|^{2} J d z d t^{\prime}+\left\|\delta_{h} \widetilde{u}\right\|_{1, \widehat{\Omega}}^{2} \\
\leq & \varepsilon\left\|\delta_{h} \widetilde{u}_{t}\right\|_{1,2,2, \widehat{\Omega}^{t}}^{2}+c\left[\| \widetilde { u } _ { t } \| _ { 1 , 2 , 2 , \widehat { \Omega } ^ { t } } ^ { 2 } \left(\varepsilon_{2} \sup _{t}\left\|\delta_{h} \widehat{\eta}\right\|_{1, \widehat{\Omega}}^{2}+c\left(\varepsilon_{2}\right) \sup _{t}\left\|\delta_{h} \widehat{\eta}\right\|_{0, \widehat{\Omega}}^{2}\right.\right. \\
& \left.+c\left(\varepsilon_{2}\right) \sup _{t}\|\widehat{\eta}\|_{0, \widehat{\Omega}}^{2}\right)+\left\|\widetilde{u}_{t}\right\|_{1,2,2, \widehat{\Omega}^{t}}^{2}\|\Phi\|_{3, \widehat{\Omega}}^{2}\left(\varepsilon_{2} \sup _{t}\|\widehat{\eta}\|_{2, \widehat{\Omega}}^{2}\right. \\
& \left.+c\left(\varepsilon_{2}\right) \sup _{t}\|\eta\|_{0, \Omega}^{2}\right)+\|\widehat{u}\|_{1,2,2, \widehat{\Omega}^{t}}^{2}+\|\Phi\|_{3, \widehat{\Omega}}^{2}\|\widetilde{u}\|_{2,2,2, \widehat{\Omega}^{t}}^{2} \\
& \left.+\|\widetilde{F}\|_{0, \widehat{\Omega}^{t}}^{2}+\|\widetilde{G}\|_{1 / 2,2,2, \widehat{S}^{t}}^{2}+\left\|v_{0}\right\|_{2, \widehat{\Omega}}^{2}\right] .
\end{aligned}
$$

Now, since by Lemma 3.2, $\left(\eta u_{t}\right)_{, t}=\eta_{t} u_{t}+\eta u_{t t} \in L_{2}\left(\Omega^{t}\right)$ and $F_{t} \in$ $L_{2}\left(\Omega^{t}\right)$ we have $[\operatorname{div} \mathbb{D}(u)]_{, t}=\operatorname{div} \mathbb{D}\left(u_{t}\right) \in L_{2}\left(\Omega^{t}\right)$. Therefore, differentiating $(3.1)_{1}$ and $(3.1)_{2}$ with respect to $t$ we obtain the problem

$$
\begin{array}{ll}
\eta u_{t t}-\operatorname{div} \mathbb{D}\left(u_{t}\right)=F_{t}-\eta_{t} u_{t} & \text { in } \Omega^{T}, \\
\mathbb{D}\left(u_{t}\right) \cdot \bar{n}_{0}=G_{t} & \text { on } S^{T}, \\
\left.u_{t}\right|_{t=0}=u_{t}(0) & \text { in } \Omega .
\end{array}
$$

Problem (3.26) is, with respect to $v=u_{t}$, of analogous form to problem (3.1), so to get an estimate for $\|v\|_{2,2,2, \Omega^{t}}^{2}=\left\|u_{t}\right\|_{2,2,2, \Omega^{t}}^{2}$ we use the same argument as in the case of the estimate for $\|u\|_{2,2,2, \Omega^{t}}^{2}$. Thus, (3.20) is now replaced by

$$
\begin{aligned}
\frac{1}{2} \frac{d}{d t} \int_{\widehat{\Omega}} \widehat{\eta}\left|\delta_{h} \widetilde{u}_{t}\right|^{2} J d z+ & \frac{\mu}{2}\left\|\delta_{h} \widetilde{u}_{t}\right\|_{1, \widehat{\Omega}}^{2} \\
\leq & c\left(\varepsilon_{2}\|\widehat{\eta}\|_{2, \widehat{\Omega}}^{2}+c\left(\varepsilon_{2}\right)\|\hat{\eta}\|_{0, \widehat{\Omega}}^{2}\right)\left\|\widetilde{u}_{t t}\right\|_{1, \widehat{\Omega}}^{2} \\
& +c\left\|\widehat{\eta}_{t}\right\|_{1, \widehat{\Omega}}^{2}\left\|\delta_{h} \widetilde{u}_{t}\right\|_{0, \widehat{\Omega}}^{2}+\varepsilon\left\|\delta_{h} \widetilde{u}_{t}\right\|_{1, \Omega}^{2}+c\left\|\widehat{u}_{t}\right\|_{1, \widehat{\Omega}}^{2} \\
& +c\left(\left\|\widetilde{F}_{t}\right\|_{0, \hat{\Omega}}^{2}+\left\|\widetilde{G}_{t}\right\|_{1 / 2, \widehat{S}}^{2}+\left\|\widehat{\eta}_{t}\right\|_{1, \widehat{\Omega}}^{2}\left\|\widetilde{u}_{t}\right\|_{1, \widehat{\Omega}}^{2}\right) .
\end{aligned}
$$

Integrating (3.27) with respect to time gives 


$$
\begin{aligned}
& \int_{\widehat{\Omega}} \widehat{\eta}\left|\delta_{h} \widetilde{u}_{t}\right|^{2} J d z+\int_{0}^{t}\left\|\delta_{h} \widetilde{u}_{t}\right\|_{1, \widehat{\Omega}}^{2} d t^{\prime} \\
& \leq c\left(\varepsilon_{2} \sup _{t}\|\widehat{\eta}\|_{2, \widehat{\Omega}}^{2}+c\left(\varepsilon_{2}\right) \sup _{t}\|\widehat{\eta}\|_{0, \widehat{\Omega}}^{2}\right)\left\|\widetilde{u}_{t t}\right\|_{1,2,2, \widehat{\Omega}^{t}}^{2} \\
&+c\left\|\widehat{\eta}_{t}\right\|_{1,2,2, \widehat{\Omega}^{t}}^{2}\left(\sup _{t}\left\|\delta_{h} \widetilde{u}_{t}\right\|_{0, \widehat{\Omega}}^{2}+\sup _{t}\left\|\widetilde{u}_{t}\right\|_{1, \widehat{\Omega}^{2}}^{2}\right)+c\left\|\widehat{u}_{t}\right\|_{1,2,2, \widehat{\Omega}^{t}}^{2} \\
&+c\left(\left\|\widetilde{F}_{t}\right\|_{0, \widehat{\Omega}^{t}}^{2}+\left\|\widetilde{G}_{t}\right\|_{1 / 2,2,2, \widehat{S}^{t}}^{2}+\int_{\widehat{\Omega}} \eta(0)\left|\delta_{h} u_{t}(0)\right|^{2} d \xi\right) .
\end{aligned}
$$

Adding inequalities (3.25) and (3.28), next going back to the old variables, summing over all neighbourhoods of the partition of unity, using the fact that $\varepsilon$ is sufficiently small and letting $h$ tend to 0 we get

$$
\begin{aligned}
& \int_{\Omega^{t}} \eta\left|u_{t \tau}\right|^{2} d \xi d t^{\prime}+\int_{\Omega} \eta\left|u_{t \tau}\right|^{2} d \xi+\left\|u_{\tau}\right\|_{1, \Omega}^{2}+\left\|u_{t \tau}\right\|_{1,2,2, \Omega^{t}} \\
& \leq c\left(\|\Phi\|_{3, \Omega}^{2}\right)\left[\left\|u_{t}\right\|_{1,2,2, \Omega^{t}}^{2}\left(\varepsilon_{1} \sup _{t}\|\eta\|_{2, \Omega}^{2}+c\left(\varepsilon_{1}\right) \sup _{t}\|\eta\|_{0, \Omega}^{2}\right)\right. \\
& \quad+\left\|u_{t t}\right\|_{1,2,2, \Omega^{t}}^{2}\left(\varepsilon_{2} \sup _{t}\|\eta\|_{2, \Omega}^{2}+c\left(\varepsilon_{2}\right) \sup _{t}\|\eta\|_{0, \Omega}^{2}\right)+\|u\|_{2,2,2, \Omega^{t}}^{2} \\
& \quad+\left\|u_{t}\right\|_{1,2,2, \Omega^{t}}^{2}+\left\|\eta_{t}\right\|_{1,2,2, \Omega^{t}}^{2} \sup _{t}\left\|u_{t}\right\|_{1, \Omega}^{2}+\|F\|_{0, \Omega^{t}}^{2}+\left\|F_{t}\right\|_{0, \Omega^{t}}^{2} \\
& \left.\quad+\|G\|_{1 / 2,2,2, S^{t}}^{2}+\left\|G_{t}\right\|_{1 / 2,2,2, S^{t}}^{2}+\left\|v_{0}\right\|_{2, \Omega}^{2}+\int_{\Omega} \eta(0)\left|u_{t \tau}(0)\right|^{2} d \xi\right] .
\end{aligned}
$$

In order to calculate the normal derivatives of $u_{t}$ we use the equation

$$
-\operatorname{div} \mathbb{D}\left(u_{t}\right)=F_{t}-\eta u_{t t}-\eta_{t} u_{t} .
$$

Hence, we have

$$
\begin{aligned}
\left\|u_{t n}\right\|_{1,2,2, \Omega^{t}}^{2} \leq & c\left[\left\|F_{t}\right\|_{0, \Omega^{t}}^{2}+\left\|u_{t \tau}\right\|_{1,2,2, \Omega^{t}}^{2}+\left\|\eta_{t}\right\|_{1,2,2, \Omega^{t}}^{2} \sup _{t}\left\|u_{t}\right\|_{1, \Omega}^{2}\right. \\
& \left.+\left(\varepsilon_{2} \sup _{t}\|\eta\|_{2, \Omega}^{2}+c\left(\varepsilon_{2}\right) \sup _{t}\|\eta\|_{0, \Omega}^{2}\right)\left\|u_{t t}\right\|_{0, \Omega^{t}}^{2}\right] .
\end{aligned}
$$

Taking into account inequalities (3.29), (3.30) and the inequality

$$
\begin{aligned}
\sup _{t}\left\|u_{n}\right\|_{1, \Omega}^{2} \leq & c\left(\sup _{t}\|F\|_{0, \Omega}^{2}+\sup _{t}\left\|u_{\tau}\right\|_{1, \Omega}^{2}\right) \\
& +c\left(\varepsilon_{1} \sup _{t}\|\eta\|_{1, \Omega}^{2}+c\left(\varepsilon_{1}\right) \sup _{t}\|\eta\|_{0, \Omega}^{2}\right) \sup _{t}\left\|u_{t}\right\|_{1, \Omega}^{2},
\end{aligned}
$$

which follows from (3.22), and using estimate (3.5) we find that $u \in L_{\infty}(0, T$; $\left.H^{2}(\Omega)\right), u_{t} \in L_{2}\left(0, T ; H^{2}(\Omega)\right)$ and

$$
\begin{aligned}
\text { (3.31) } & \|u\|_{2, \Omega}^{2}+\left\|u_{t}\right\|_{2,2,2, \Omega^{t}}^{2} \\
\leq & \Psi_{5}\left(1 / \varrho_{*}, \varrho^{*}, t,\left\|\eta_{t}\right\|_{1,2,2, \Omega^{t}}^{2},\left\|\eta_{t t}\right\|_{0, \Omega^{t}}^{2}, \varepsilon_{2} \sup _{t}\|\eta\|_{2, \Omega}^{2}+c\left(\varepsilon_{2}\right) \sup _{t}\|\eta\|_{0, \Omega}^{2}\right) \\
\times & {\left[\|F\|_{0, \Omega^{t}}^{2}+\left\|F_{t}\right\|_{0, \Omega^{t}}^{2}+\varepsilon_{1}\left\|F_{t t}\right\|_{0, \Omega^{t}}^{2}+\sup _{t}\|F\|_{0, \Omega}^{2}\right.} \\
+ & \left.\|G\|_{1 / 2,2,2, S^{t}}^{2}+\left\|G_{t}\right\|_{1 / 2,2,2, S^{t}}^{2}+\varepsilon_{1}\left\|G_{t t}\right\|_{0, S^{t}}^{2}+|u(0)|_{2,0, \Omega}^{2}\right],
\end{aligned}
$$

where $\Psi_{5}$ is a positive increasing continuous function. 
It remains to find an estimate for $\|u\|_{3,2,2, \Omega^{t}}^{2}$. Using the same argument as in [6] and after similar calculations to those for (A.16) of [6] we get

$$
\begin{aligned}
& \quad \frac{1}{2} \frac{d}{d t} \int_{\widehat{\Omega}} \widehat{\eta}\left|\partial_{\tau}^{2} j_{\delta} u\right|^{2} J d z+\frac{\mu}{2}\left\|\partial_{\tau}^{2} j_{\delta} \widetilde{u}\right\|_{1, \widehat{\Omega}}^{2} \\
& \leq c\left[\|\widehat{\eta}\|_{2, \widehat{\Omega}}^{2}\left\|j_{\delta} \widetilde{u}_{t}\right\|_{1, \widehat{\Omega}}^{2}+\left(\varepsilon_{2}\left\|\widehat{\eta}_{t}\right\|_{1, \widehat{\Omega}}^{2}+c\left(\varepsilon_{2}\right)\left\|\widehat{\eta}_{t}\right\|_{0, \widehat{\Omega}}^{2}\right)\left\|\partial_{\tau}^{2} j_{\delta} \widetilde{u}\right\|_{0, \widehat{\Omega}}^{2}\right. \\
& \left.\quad+\|\widehat{\eta}\|_{2, \widehat{\Omega}}^{2}\left\|\widetilde{u}_{t}\right\|_{1, \widehat{\Omega}}^{2}+\|\widehat{u}\|_{2, \widehat{\Omega}}^{2}+\|\widetilde{F}\|_{1, \widehat{\Omega}}^{2}+\|\widetilde{G}\|_{3 / 2, \widehat{S}}^{2}+\varepsilon\left\|\partial_{n}^{2} \partial_{\tau} j_{\delta} \widetilde{u}\right\|_{0, \widehat{\Omega}}^{2}\right],
\end{aligned}
$$

where $j_{\delta}$ is the Friedrichs mollifier operator. Integrating (3.32) with respect to time yields

$$
\int_{\widehat{\Omega}} \widehat{\eta}\left|\partial_{\tau}^{2} j_{\delta} \widetilde{u}\right|^{2} J d z+\mu\left\|\partial_{\tau}^{2} j_{\delta} \widetilde{u}\right\|_{1,2,2, \hat{\Omega}^{t}}^{2}
$$

$$
\begin{aligned}
\leq & c\left[\|\widehat{\eta}\|_{2,2,2, \widehat{\Omega}^{t}}^{2} \sup _{t}\left\|\widetilde{u}_{t}\right\|_{1, \widehat{\Omega}}^{2}\right. \\
& +\left(\varepsilon_{2} \sup _{t}\left\|\widehat{\eta}_{t}\right\|_{1, \widehat{\Omega}}^{2}+c\left(\varepsilon_{2}\right) \sup _{t}\left\|\widehat{\eta}_{t}\right\|_{0, \widehat{\Omega}}^{2}\right)\|\widehat{u}\|_{2,2,2, \widehat{\Omega}^{t}}^{2}+\|\widehat{u}\|_{2,2,2, \widehat{\Omega}^{t}}^{2} \\
& \left.+\|\widetilde{F}\|_{1,2,2, \widehat{\Omega}^{t}}^{2}+\|\widetilde{G}\|_{3 / 2,2,2, \widehat{S}^{t}}^{2}+\varepsilon\left\|\partial_{n}^{2} \partial_{\tau} j_{\delta} \widetilde{u}\right\|_{0, \widehat{\Omega}^{t}}^{2}+\int_{\widehat{\Omega}} \widehat{\eta}(0)\left|\partial_{\tau}^{2} \widetilde{u}(0)\right|^{2} J d z\right] .
\end{aligned}
$$

Next, (A.18) of [6] is now replaced by

$$
\left\|\partial_{\tau} \partial_{n}^{2} \int \omega_{\delta}(x-y)\left[1-\frac{1}{\Phi_{3, x}^{2}}\left(\Phi_{3, x}^{2}-\Phi_{3, y}^{2}\right)\right] \widetilde{u}(y) d y\right\|_{0, \hat{\Omega}^{t}}^{2}
$$

$$
\leq c\|\widehat{r}\|_{2,2,2, \widehat{\Omega}^{t}}^{2} \sup _{t}\left\|\widetilde{u}_{t}\right\|_{1, \widehat{\Omega}^{2}}^{2}+c_{1}\left\|\partial_{\tau}^{2} j_{\delta} \widetilde{u}\right\|_{1,2,2, \widehat{\Omega}^{t}}^{2}+c\|\widehat{u}\|_{2,2,2, \widehat{\Omega}^{t}}^{2}+c\|\widetilde{F}\|_{1,2,2, \widehat{\Omega}^{t}}^{2},
$$

where $\omega_{\delta}$ is the smooth kernel of the mollifier operator $j_{\delta}$. Estimates (3.33) and (3.34) imply

$$
\begin{aligned}
& \frac{2 c_{1}}{\mu} \int_{\widehat{\Omega}}\left|\partial_{\tau}^{2} j_{\delta} \widetilde{u}\right|^{2} J d z+c_{1}\left\|\partial_{\tau}^{2} j_{\delta} \widetilde{u}\right\|_{1,2,2, \widehat{\Omega}^{t}}^{2} \\
& \quad+\left\|\partial_{\tau} \partial_{n}^{2} \int \omega_{\delta}(x-y)\left[1-\frac{1}{\Phi_{3, x}^{2}}\left(\Phi_{3, x}^{2}-\Phi_{3, y}^{2}\right)-\frac{2 \varepsilon c_{1}}{\mu}\right] \widetilde{u}(y) d y\right\|_{0, \widehat{\Omega}^{t}}^{2} \\
& \leq c\left[\|\widehat{\eta}\|_{2,2,2, \widehat{\Omega}^{t}}^{2} \sup _{t}\left\|\widetilde{u}_{t}\right\|_{1, \widehat{\Omega}}^{2}\right. \\
& \quad+\left(\varepsilon_{2} \sup _{t}\left\|\widehat{\eta}_{t}\right\|_{1, \widehat{\Omega}}^{2}+c\left(\varepsilon_{2}\right) \sup _{t}\left\|\widehat{\eta}_{t}\right\|_{0, \widehat{\Omega}}^{2}\right)\|\widehat{u}\|_{2,2,2, \widehat{\Omega}^{t}}^{2} \\
& \quad+\|\widehat{u}\|_{2,2,2, \widehat{\Omega}^{t}}^{2}+\|\widetilde{F}\|_{1,2,2, \widehat{\Omega}^{t}}^{2}+\|\widetilde{G}\|_{3 / 2,2,2, \widehat{S}^{t}}^{2} \\
& \left.\quad+\varepsilon\left\|\partial_{n}^{2} \partial_{\tau} j_{\delta} \widetilde{u}\right\|_{0, \widehat{\Omega}^{t}}^{2}+\int_{\widehat{\Omega}} \widehat{\eta}(0)\left|\partial_{\tau}^{2} \widetilde{u}(0)\right|^{2} J d z\right] .
\end{aligned}
$$

Using the fact that $\Phi_{3, x}^{2}$ is close to one and $\Phi_{3, x}^{2}-\Phi_{3, y}^{2}$ is close to zero for $x, y \in \widehat{\Omega}$ and for $\widehat{\Omega}$ sufficiently small, we obtain from (3.35), after taking the 
limit as $\delta \rightarrow 0$,

$$
\begin{aligned}
& \int_{\widehat{\Omega}} \widehat{\eta}\left|\partial_{\tau}^{2} \widetilde{u}\right|^{2} J d z+\left\|\partial_{\tau}^{2} \widetilde{u}\right\|_{1,2,2, \widehat{\Omega}^{t}}^{2}+\left\|\partial_{n}^{2} \partial_{\tau} \widetilde{u}\right\|_{0, \widehat{\Omega}^{t}}^{2} \\
\leq & c\left[\|\widehat{\eta}\|_{2,2,2, \widehat{\Omega}^{t}}^{2} \sup _{t}\left\|\widetilde{u}_{t}\right\|_{1, \widehat{\Omega}}^{2}\right. \\
& +\left(\varepsilon_{2} \sup _{t}\left\|\widehat{\eta}_{t}\right\|_{1, \widehat{\Omega}^{2}}^{2}+c\left(\varepsilon_{2}\right) \sup _{t}\left\|\widehat{\eta}_{t}\right\|_{0, \widehat{\Omega}}^{2}\right)\|\widehat{u}\|_{2,2,2, \widehat{\Omega}^{t}}^{2} \\
& \left.+\|\widehat{u}\|_{2,2,2, \widehat{\Omega}^{t}}^{2}+\|\widetilde{F}\|_{1,2,2, \widehat{\Omega}^{t}}^{2}+\|\widetilde{G}\|_{3 / 2,2,2, \widehat{S}^{t}}^{2}+\int_{\widehat{\Omega}} \widehat{\eta}(0)\left|\partial_{\tau}^{2} \widetilde{u}(0)\right|^{2} J d z\right] .
\end{aligned}
$$

Next, from equation (A.13) of [6] we have

$$
\begin{aligned}
\left\|\partial_{n}^{3} \widetilde{u}\right\|_{0, \widehat{\Omega}^{t}}^{2} \leq & c\left(\left\|\partial_{n}^{2} \partial_{\tau} \widetilde{u}\right\|_{0, \widehat{\Omega}^{t}}^{2}+\left\|\partial_{n} \partial_{\tau}^{2} \widetilde{u}\right\|_{0, \widehat{\Omega}^{t}}^{2}\right. \\
& \left.+\|\widehat{u}\|_{2,2,2, \widehat{\Omega}^{t}}^{2}+\|\widehat{\eta}\|_{2,2,2, \widehat{\Omega}^{t}}^{2} \sup _{t}\left\|\widetilde{u}_{t}\right\|_{1, \widehat{\Omega}^{t}}^{2}+\|\widetilde{F}\|_{1,2,2, \widehat{\Omega}^{t}}^{2}\right) .
\end{aligned}
$$

Taking into account (3.36) and (3.37) we see that $u \in L_{2}\left(0, T ; H^{3}(\Omega)\right)$ and

$$
\begin{aligned}
\|u\|_{3,2,2, \Omega^{t}}^{2} \leq & c\left[\|\eta\|_{2,2,2, \Omega^{t}}^{2} \sup _{t}\left\|u_{t}\right\|_{1, \Omega}^{2}\right. \\
& +\left(\varepsilon_{2} \sup _{t}\left\|\eta_{t}\right\|_{1, \Omega}^{2}+c\left(\varepsilon_{2}\right) \sup _{t}\left\|\eta_{t}\right\|_{0, \Omega}^{2}\right)\|u\|_{2,2,2, \Omega^{t}}^{2} \\
& +\|u\|_{2,2,2, \Omega^{t}}^{2}+\|F\|_{1,2,2, \Omega^{t}}^{2} \\
& \left.+\|G\|_{3 / 2,2,2, S^{t}}^{2}+\int_{\Omega} \eta(0)\left|\partial_{\tau}^{2} u(0)\right|^{2} d z\right] .
\end{aligned}
$$

Adding inequalities (3.31), (3.38) and (3.5) we obtain estimate (3.15).

To complete the proof notice that from $u \in L_{\infty}\left(0, T ; H^{2}(\Omega)\right) \cap L_{2}(0, T$; $\left.H^{3}(\Omega)\right)$ and $u_{t} \in L_{\infty}\left(0, T ; H^{1}(\Omega)\right) \cap L_{2}\left(0, T ; H^{2}(\Omega)\right)$ it follows that $u \in$ $C\left(0, T ; H^{2}(\Omega)\right)$. Next, since $u_{t} \in L_{\infty}\left(0, T ; H^{1}(\Omega)\right) \cap L_{2}\left(0, T ; H^{2}(\Omega)\right)$ and $u_{t t} \in L_{\infty}\left(0, T ; L_{2}(\Omega)\right) \cap L_{2}\left(0, T ; H^{1}(\Omega)\right)$ we have $u_{t} \in C\left(0, T ; H^{1}(\Omega)\right)$.

In order to prove that $u_{t t} \in C\left(0, T ; L_{2}(\Omega)\right)$ differentiate $(3.1)_{1}$ with respect to $t$, multiply by $\phi \in H^{1}(\Omega)$ and integrate over $\Omega$, using the boundary condition $(3.1)_{2}$, to get

$$
\begin{aligned}
\int_{\Omega} u_{t t} \phi d \xi= & \int_{\Omega}\left(-\frac{\eta_{t} u_{t}}{\eta}+\frac{F_{t}}{\eta}-\nabla\left(\frac{1}{\eta}\right) \mathbb{D}\left(u_{t}\right)\right) \phi d \xi \\
& -\int_{\Omega} \frac{\mathbb{D}\left(u_{t}\right)}{\eta} \nabla \phi d \xi+\int_{S} \frac{G_{t}}{\eta} \phi d \xi_{s} \quad \forall \phi \in H^{1}(\Omega),
\end{aligned}
$$

where $\mathbb{D}\left(u_{t}\right) \nabla \phi=\sum_{i, j=1}^{3} D_{i j}\left(u_{t}\right) \phi_{i x_{j}}$. Hence, 


$$
\begin{aligned}
\frac{d}{d t} \int_{\Omega} u_{t t} \phi d \xi= & \int_{\Omega}\left[-\nabla\left(\left(\frac{1}{\eta}\right)_{, t}\right) \mathbb{D}\left(u_{t}\right)-\nabla\left(\frac{1}{\eta}\right) \mathbb{D}\left(u_{t t}\right)\right. \\
& \left.-\frac{\eta_{t t} u_{t}}{\eta}-\frac{\eta_{t} u_{t t}}{\eta}-\left(\frac{1}{\eta}\right)_{, t} \eta_{t} u_{t}+\frac{F_{t t}}{\eta}+F_{t}\left(\frac{1}{\eta}\right)_{, t}\right] \phi d \xi \\
& -\int_{\Omega}\left[\frac{\mathbb{D}\left(u_{t t}\right)}{\eta}-\mathbb{D}\left(u_{t}\right)\left(\frac{1}{\eta}\right)_{, t}\right] \nabla \phi d \xi \\
& +\int_{S}\left(\frac{G_{t t}}{\eta}+G_{t}\left(\frac{1}{\eta}\right)_{, t}\right) \phi d \xi_{s} \\
\equiv & \left\langle g_{1}, \phi\right\rangle_{\Omega}+\left\langle g_{2}, \phi\right\rangle_{S} \quad \forall \phi \in H^{1}(\Omega) .
\end{aligned}
$$

Identity (3.39) implies

$$
\begin{aligned}
& \left\|u_{t t t}\right\|_{L_{2}\left(0, T ;\left(H^{1}(\Omega)\right)^{*}\right)}^{2} \\
& \quad \leq c \int_{0}^{T} \sup _{\|\phi\|_{1, \Omega} \leq 1}\left|\left\langle g_{1}, \phi\right\rangle_{\Omega}\right|^{2} d t+c \int_{0}^{T} \sup _{\|\phi\|_{1, \Omega} \leq 1}\left|\left\langle g_{2}, \phi\right\rangle_{S}\right|^{2} d t \\
& \quad \leq \Psi_{6}\left(\|\eta\|_{\mathcal{B}_{T}}^{2},\|1 / \eta\|_{\mathcal{B}_{T}}^{2},\left\|u_{t}\right\|_{2,2,2, \Omega^{T}}^{2},\left\|u_{t t}\right\|_{1,2,2, \Omega^{T}}^{2},\left\|G_{t t}\right\|_{0, S^{T}}^{2}\right. \\
& \left.\quad\left\|G_{t}\right\|_{1 / 2,2,2, S^{T}},\left\|F_{t t}\right\|_{0, \Omega^{T}}^{2},\left\|F_{t}\right\|_{0, \Omega^{T}}^{2}\right),
\end{aligned}
$$

where $\Psi_{6}$ is a positive increasing continuous function of its arguments. Hence $u_{t t} \in C\left(0, T ; L_{2}(\Omega)\right)$. This completes the proof of the lemma.

Now, consider the problem

$$
\begin{array}{ll}
\eta u_{t}-\operatorname{div}_{w} \mathbb{D}_{w}(u)=F & \text { in } \Omega^{T}, \\
\mathbb{D}_{w}(u) \cdot \bar{n}_{w}=G & \text { on } S^{T}, \\
\left.u\right|_{t=0}=v_{0} & \text { in } \Omega,
\end{array}
$$

where $\eta$ and $w$ are given functions, $\bar{n}_{w}=\bar{n}\left(X_{w}(\xi, t), t\right), \mathbb{D}_{w}(u)=\mu \mathbb{S}_{w}(u)+$ $(\nu-\mu) I \operatorname{div}_{w} u, \mathbb{S}_{w}(u)=\left\{\partial_{x_{i}} \xi_{k} \partial_{\xi_{k}} u_{j}+\partial_{x_{j}} \xi_{k} \partial_{\xi_{k}} u_{i}\right\}_{i, j=1,2,3}, I=\left\{\delta_{i j}\right\}_{i, j=1,2,3}$. We assume that $\eta$ satisfies $(3.2)$ and $w=w(\xi, t)$ is such that

$$
x=\xi+\int_{0}^{t} w(\xi, s) d s \equiv X_{w}(\xi, t)=x(\xi, t)
$$

and $x_{\xi}=\left\{\partial x_{i} / \partial \xi_{j}\right\}_{i, j=1,2,3}, \xi_{x}=\left\{\partial \xi_{i} / \partial x_{j}\right\}_{i, j=1,2,3}$ are matrices with determinants close to 1 for $t \in[0, T]$.

In order to prove the existence of solutions of (3.40) consider first the problem

$$
\begin{array}{ll}
\eta u_{t}-\operatorname{div} \mathbb{D}(u)=\operatorname{div}_{w} \mathbb{D}_{w}\left(u^{*}\right)-\operatorname{div} \mathbb{D}\left(u^{*}\right)+F & \text { in } \Omega^{T}, \\
\mathbb{D}(u) \cdot \bar{n}_{0}=\mathbb{D}\left(u^{*}\right) \cdot \bar{n}_{0}-\mathbb{D}_{w}\left(u^{*}\right) \cdot \bar{n}_{w}+G & \text { on } S^{T}, \\
\left.u\right|_{t=0}=v_{0} & \text { in } \Omega,
\end{array}
$$

where $u^{*}$ is a given function. 
LEMMA 3.4. Let the assumptions of Lemma 3.3 be satisfied and let $w, u^{*}$ $\in \mathcal{A}_{T}$, where $\mathcal{A}_{T}$ is given by (2.1). Moreover, assume that

$$
\sup _{t \in[0, T]} \sup _{\xi \in \Omega}\left|I-x_{\xi}\right| \leq \delta
$$

and

$$
\sup _{t \in[0, T]} \sup _{\xi \in \Omega}\left|I-\xi_{x}\right| \leq \delta
$$

where $\delta$ is sufficiently small and $I$ is the unit matrix. Then there exists a unique solution $u \in \mathcal{A}_{T}$ of problem (3.41) and for $t \leq T$ the following estimate holds:

$$
\begin{aligned}
\|u\|_{\mathcal{A}_{t}}^{2} \leq & \Psi_{7}\left(1 / \varrho_{*}, \varrho^{*}, t, h\left(t, \eta, \varepsilon_{2}\right)\right)\left[\|F\|_{1,2,2, \Omega^{t}}^{2}+\left\|F_{t}\right\|_{0, \Omega^{t}}^{2}\right. \\
& +\varepsilon_{1}\left\|F_{t t}\right\|_{0, \Omega^{t}}^{2}+\sup _{t}\|F\|_{0, \Omega}^{2}+\|G\|_{3 / 2,2,2, S^{t}}^{2} \\
& +\left\|G_{t}\right\|_{1 / 2,2,2, S^{t}}^{2}+\varepsilon_{1}\left\|G_{t t}\right\|_{0, S^{t}}^{2}+\left.\mathbf{|} u(0)\right|_{2,0, \Omega} ^{2} \\
& \left.+c\left(t, u^{*}, \varepsilon_{4}\right) \Psi_{8}\left(a(w, t), b\left(t, w, \varepsilon_{3}\right)\right)\right]
\end{aligned}
$$

where

$$
\begin{aligned}
a(w, t)= & t \int_{0}^{t}\|w\|_{3, \Omega}^{2} d t^{\prime} \\
b\left(t, w, \varepsilon_{3}\right)= & \int_{0}^{t}\left(\varepsilon_{3}\|w\|_{3, \Omega}^{2}+c\left(\varepsilon_{3}\right)\|w\|_{0, \Omega}^{2}\right) d t^{\prime} \\
& +\int_{0}^{t}\left(\varepsilon_{3}\left\|w_{t}\right\|_{2, \Omega}^{2}+c\left(\varepsilon_{3}\right)\left\|w_{t}\right\|_{0, \Omega}^{2}\right) d t^{\prime} \\
& +\left(\varepsilon_{3} \sup _{t}\|w\|_{2, \Omega}^{2}+c\left(\varepsilon_{3}\right) \sup _{t}\|w\|_{0, \Omega}^{2}\right) \\
& +\left(\varepsilon_{3} \sup _{t}\left\|w_{t}\right\|_{1, \Omega}^{2}+c\left(\varepsilon_{3}\right) \sup _{t}\left\|w_{t}\right\|_{0, \Omega}^{2}\right), \\
c\left(t, u^{*}, \varepsilon_{4}\right)= & \int_{0}^{t}\left(\varepsilon_{4}\left\|u^{*}\right\|_{3, \Omega}^{2}+c\left(\varepsilon_{4}\right)\left\|u^{*}\right\|_{0, \Omega}^{2}\right) d t^{\prime} \\
& +\int_{0}^{t}\left(\varepsilon_{4}\left\|u_{t}^{*}\right\|_{2, \Omega}^{2}+c\left(\varepsilon_{4}\right)\left\|u_{t}^{*}\right\|_{0, \Omega}^{2}\right) d t^{\prime}+t \int_{0}^{t}\left\|u^{*}\right\|_{3, \Omega}^{2} d t^{\prime},
\end{aligned}
$$

$\varepsilon_{i} \in(0,1)(i=1, \ldots, 4)$ are constants, $\Psi_{7}$ and $\Psi_{8}$ are positive increasing continuous functions of their arguments $\left(\Psi_{7}\right.$ also depends on $\left.\|\Phi\|_{3, \Omega}^{2}\right)$ and $h\left(t, \eta, \varepsilon_{2}\right)$ is given by (3.16).

Proof. In the case of smooth functions $w, u^{*}$ and $\Phi$ the existence of a unique solution $u \in \mathcal{A}_{T}$ of problem (3.41) follows from Lemmas 3.2 and 3.3. Estimate (3.44) is obtained in the same way as (3.5) and (3.15). We shall show for example how to obtain an estimate for $\sup _{t}\left\|u_{t}\right\|_{1, \Omega}^{2}$. Differentiating 
the system (3.40) with respect to $t$ we get

$$
\begin{aligned}
& \eta u_{t t}-\operatorname{div} \mathbb{D}\left(u_{t}\right)=-\eta_{t} u_{t}+\operatorname{div}_{w} \mathbb{D}_{w}\left(u_{t}^{*}\right) \\
& -\operatorname{div} \mathbb{D}\left(u_{t}^{*}\right)+F_{1}+F_{t} \quad \text { in } \Omega^{T}, \\
& \mathbb{D}\left(u_{t}\right) \cdot \bar{n}_{0}=\mathbb{D}\left(u_{t}^{*}\right) \cdot \bar{n}_{0}-\mathbb{D}_{w}\left(u_{t}^{*}\right) \cdot \bar{n}_{w}+G_{1}+G_{t} \quad \text { on } S^{T},
\end{aligned}
$$

where $F_{1}$ and $G_{1}$ are functions satisfying

$$
\begin{aligned}
& \left|F_{1}\right| \leq\left|f_{1}\right|\left|w_{\xi}\right|\left|u_{\xi \xi}^{*}\right|+\left|f_{2}\right|\left|w_{\xi}\right|\left|\int_{0}^{t} w_{\xi \xi} d t^{\prime}\right|\left|u_{\xi}^{*}\right|+\left|f_{3}\right|\left|w_{\xi \xi}\right|\left|u_{\xi}^{*}\right|, \\
& \left|G_{1}\right| \leq\left|g_{1}\right|\left|w_{\xi}\right|\left|u_{\xi}^{*}\right|+\left|g_{2}\right|\left|w_{\xi}\right|\left|\int_{0}^{t} w_{\xi} d t^{\prime}\right|\left|u_{\xi}^{*}\right|
\end{aligned}
$$

and $f_{i}(i=1,2,3)$ and $g_{i}(i=1,2)$ are $C^{\infty}$ functions of $I+\int_{0}^{t} w_{\xi} d t^{\prime}$.

Now, multiplying $(3.48)_{1}$ by $u_{t} J_{w}$ (where $J_{w}$ is the Jacobian of the transformation $x=X_{w}(\xi, t)$ ), integrating over $\Omega^{t}$, using the boundary condition $(3.48)_{2}$ and the Korn inequality yields

$$
\begin{aligned}
& \int_{\Omega^{t}} \eta u_{t t}^{2} J_{w} d \xi d t^{\prime}+c\left\|u_{t}\right\|_{1, \Omega}^{2} \\
& \leq \varepsilon\left\|u_{t t}\right\|_{1,2,2, \Omega^{t}}^{2}+\varepsilon b\left(t, w, \varepsilon_{3}\right) \sup _{t}\left\|u_{t}\right\|_{1, \Omega}^{2} \\
& \quad+c\left(\left\|\eta_{t}\right\|_{1,2,2, \Omega^{t}}^{2} \sup _{t}\left\|u_{t}\right\|_{0, \Omega}^{2}+\left\|u_{t}\right\|_{1,2,2, \Omega^{t}}^{2}+\left\|F_{t}\right\|_{0, \Omega^{t}}^{2}+\left\|G_{t}\right\|_{0, S^{t}}^{2}\right) \\
& \quad+c\left[\frac{\mu}{2} \int_{\Omega^{t}}\left|\mathbb{S}_{w}\left(u_{t}^{*}\right) \mathbb{S}_{w}\left(u_{t t}\right)-\mathbb{S}\left(u_{t}^{*}\right) \mathbb{S}\left(u_{t t}\right)\right| d \xi d t^{\prime}\right. \\
& \left.\quad+(\nu-\mu) \int_{\Omega^{t}}\left|\operatorname{div}_{w} u_{t}^{*} \operatorname{div}_{w} u_{t t}-\operatorname{div} u_{t}^{*} \operatorname{div} u_{t t}\right| d \xi d t^{\prime}\right] \\
& \quad+c \int_{\Omega^{t}}\left|F_{1} u_{t t}\right| d \xi d t^{\prime}+c \int_{S^{t}}\left|G_{1} u_{t t}\right| d \xi_{S} d t^{\prime}+\left\|u_{t \xi}(0)\right\|_{0, \Omega}^{2} .
\end{aligned}
$$

By (3.49)-(3.51) and Lemma 2.1,

$$
\begin{aligned}
& \int_{\Omega^{t}}\left|F_{1} u_{t t}\right| d \xi d t^{\prime}+\int_{S^{t}}\left|G_{1} u_{t t}\right| d \xi_{s} d t^{\prime} \\
\leq & \varepsilon\left\|u_{t t}\right\|_{1,2,2, \Omega^{t}}^{2}+c(\varepsilon)\left(\varepsilon_{3} \sup _{t}\|w\|_{2, \Omega}^{2}++c\left(\varepsilon_{3}\right) \sup _{t}\|w\|_{0, \Omega}^{2}\right) \\
& \times\left(\varepsilon_{4} \int_{0}^{t}\left\|u^{*}\right\|_{3, \Omega}^{2} d t^{\prime}+c\left(\varepsilon_{4}\right) \int_{0}^{t}\left\|u^{*}\right\|_{0, \Omega}^{2} d t^{\prime}\right) \\
& \times\left(1+t \int_{0}^{t}\|w\|_{3, \Omega}^{2} d t^{\prime}\right)+c(\varepsilon)\left(\varepsilon_{3} \int_{0}^{t}\|w\|_{3, \Omega}^{2} d t^{\prime}+c\left(\varepsilon_{3}\right) \int_{0}^{t}\|w\|_{0, \Omega}^{2} d t^{\prime}\right) \\
& \times\left(\varepsilon_{4} \sup _{t}\left\|u^{*}\right\|_{2, \Omega}^{2}+c\left(\varepsilon_{4}\right) \sup _{t}\left\|u^{*}\right\|_{0, \Omega}^{2}\right)
\end{aligned}
$$


and

$$
\begin{aligned}
\frac{\mu}{2} \int_{\Omega^{t}}\left|\mathbb{S}_{w}\left(u_{t}^{*}\right) \mathbb{S}_{w}\left(u_{t t}\right)-\mathbb{S}\left(u_{t}^{*}\right) \mathbb{S}\left(u_{t t}\right)\right| d \xi d t^{\prime} & \\
& +(\nu-\mu) \int_{\Omega^{t}}\left|\operatorname{div}_{w} u_{t}^{*} \operatorname{div}_{w} u_{t t}-\operatorname{div} u_{t}^{*} \operatorname{div} u_{t t}\right| d \xi d t^{\prime} \\
\leq & \delta c\left(\varepsilon_{4}\right)\left\|u_{t t}\right\|_{1, \Omega^{t}}^{2}+\varepsilon_{4}\left\|u_{t}^{*}\right\|_{1, \Omega^{t}}^{2} .
\end{aligned}
$$

In view of (3.52)-(3.53) inequality (3.51) gives

$$
\begin{aligned}
& \int_{\Omega^{t}} \eta u_{t t}^{2} J_{w} d \xi d t^{\prime}+\left\|u_{t}\right\|_{1, \Omega}^{2} \\
& \leq \varepsilon_{5}\left\|u_{t t}\right\|_{1,2,2, \Omega^{t}}^{2}+\varepsilon b\left(t, w, \varepsilon_{3}\right) \sup _{t}\left\|u_{t}\right\|_{1, \Omega}^{2} \\
& \quad+c\left(\left\|F_{t}\right\|_{0, \Omega^{t}}^{2}+\left\|G_{t}\right\|_{0, S^{t}}^{2}+\left\|u_{t}\right\|_{1,2,2, \Omega^{t}}^{2}+\left\|\eta_{t}\right\|_{1,2,2, \Omega^{t}}^{2} \sup _{t}\left\|u_{t}\right\|_{0, \Omega}^{2}\right) \\
& \quad+\left\|u_{t \xi}(0)\right\|_{0, \Omega}^{2}+c\left(t, u^{*}, \varepsilon_{4}\right) \Psi_{9}\left(a(w, t), b\left(t, w, \varepsilon_{3}\right)\right),
\end{aligned}
$$

where $\varepsilon_{5}>0$ is sufficiently small, and $\Psi_{9}$ is a positive increasing continuous function.

To estimate $\left\|u_{t t}\right\|_{1,2,2, \Omega^{t}}^{2}$ differentiate (3.48) ${ }_{1}$ twice with respect to $t$, multiply the result by $u_{t t} J_{w}$, and integrate over $\Omega$. Using the boundary condition $(3.48)_{2}$ and the Korn inequality we obtain

$$
\begin{aligned}
\int_{\Omega} \eta u_{t t}^{2} J_{w} d \xi & +\left\|u_{t t}\right\|_{1,2,2, \Omega^{t}}^{2} \\
\leq & \Psi_{10}\left(1 / \varrho_{*}, \varrho^{*}, t,\left\|\eta_{t}\right\|_{1,2,2, \Omega^{t}}^{2}\right)\left[\varepsilon_{1}\left\|F_{t t}\right\|_{0, \Omega^{t}}^{2}+\varepsilon_{1}\left\|G_{t t}\right\|_{0, \Omega^{t}}^{2}\right. \\
& +\int_{\Omega} \eta(0) u_{t t}^{2}(0) d \xi+\sup _{t}\left\|u_{t}\right\|_{1, \Omega}^{2}\left\|\eta_{t t}\right\|_{0, \Omega^{t}}^{2} \\
& \left.+\left\|u_{t t}\right\|_{0, \Omega^{t}}^{2}+c\left(t, u^{*}, \varepsilon_{4}\right) \Psi_{11}\left(a(w, t), b\left(t, w, \varepsilon_{3}\right)\right)\right],
\end{aligned}
$$

where $\Psi_{10}$ and $\Psi_{11}$ are positive increasing continuous functions.

In the same way we get the estimate

$$
\begin{aligned}
\int_{\Omega} \eta u_{t}^{2} J_{w} d \xi+ & \left\|u_{t}\right\|_{1,2,2, \Omega^{t}}^{2} \\
\leq & \Psi_{12}\left(1 / \varrho_{*}, \varrho^{*}, t,\left\|\eta_{t}\right\|_{1,2,2, \Omega^{t}}^{2}\right)\left[\left\|F_{t}\right\|_{0, \Omega^{t}}^{2}+\left\|G_{t}\right\|_{0, S^{t}}^{2}\right. \\
& \left.+\left\|u_{t}(0)\right\|_{0, \Omega}^{2}+c\left(t, u^{*}, \varepsilon_{4}\right) \Psi_{13}\left(a(w, t), b\left(t, w, \varepsilon_{3}\right)\right)\right],
\end{aligned}
$$

where $\Psi_{12}$ and $\Psi_{13}$ are positive increasing continuous functions. 
Now, taking into account (3.54)-(3.56) we have

$$
\begin{aligned}
&\left\|u_{t t}\right\|_{0, \Omega}^{2}+\left\|u_{t t}\right\|_{1,2,2, \Omega^{t}}^{2}+\left\|u_{t}\right\|_{1, \Omega}^{2}+\left\|u_{t}\right\|_{1,2,2, \Omega^{t}}^{2} \\
& \leq \Psi_{14}\left(1 / \varrho_{*}, \varrho^{*}, t,\left\|\eta_{t}\right\|_{1,2,2, \Omega^{t}}^{2}\left[\left\|F_{t}\right\|_{0, \Omega^{t}}^{2}+\varepsilon_{1}\left\|F_{t t}\right\|_{0, \Omega^{t}}^{2}\right.\right. \\
&+\left\|G_{t}\right\|_{0, S^{t}}^{2}+\varepsilon_{1}\left\|G_{t t}\right\|_{0, S^{t}}^{2}+\left\|u_{t}(0)\right\|_{0, \Omega}^{2}+\left\|u_{t t}(0)\right\|_{0, \Omega}^{2} \\
&\left.+c\left(t, u^{*}, \varepsilon_{4}\right) \Psi_{15}\left(a(w, t), b\left(t, w, \varepsilon_{3}\right)\right)\right],
\end{aligned}
$$

where $\Psi_{14}$ and $\Psi_{15}$ are positive increasing continuous functions.

Estimates for $\|u\|_{3,2,2, \Omega^{t}}^{2},\|u\|_{2, \Omega}^{2},\left\|u_{t}\right\|_{2,2,2, \Omega^{t}}^{2}$ are obtained by using similar considerations to those above and in Lemmas 3.2-3.3. Hence, estimate (3.44) holds.

In the case when $u^{*}, w \in \mathcal{A}_{T}$ and $\Phi \in H^{3}(\Omega)$ we get the existence of a solution $u \in \mathcal{A}_{T}$ of problem (3.41) and estimate (3.44) by using the density of smooth functions in $\mathcal{A}_{T}$ and in $H^{3}(\Omega)$, respectively. This completes the proof of the lemma.

Lemma 3.5. Let the assumptions of Lemma 3.3 and (3.42)-(3.43) be satisfied. Let $w \in \mathcal{A}_{T}$ (where $\mathcal{A}_{T}$ is given by (2.1)). Then there exists $T_{*}>0$ (depending on the arguments of $\Psi_{16}$ and on the expression in square brackets on the right-hand side of (3.57)) such that for $T \leq T_{*}$ there exists a unique solution $u \in \mathcal{A}_{T}$ of problem (3.40) and for $t \leq T$ the following estimate holds:

$$
\begin{aligned}
\|u\|_{\mathcal{A}_{t}}^{2} \leq & \Psi_{16}\left(1 / \varrho_{*}, \varrho^{*}, t, h\left(t, \eta, \varepsilon_{2}\right), a(w, t), b\left(t, w, \varepsilon_{3}\right)\right) \\
& \times\left[\|F\|_{1,2,2, \Omega^{t}}^{2}+\left\|F_{t}\right\|_{0, \Omega^{t}}^{2}+\varepsilon_{1}\left\|F_{t t}\right\|_{0, \Omega^{t}}^{2}+\sup _{t}\|F\|_{0, \Omega}^{2}\right. \\
& +\|G\|_{3 / 2,2,2, S^{t}}^{2}+\left\|G_{t}\right\|_{1 / 2,2,2, S^{t}}^{2} \\
& \left.+\varepsilon_{1}\left\|G_{t t}\right\|_{0, S^{t}}^{2}+\sup _{t}\|G\|_{0, S}^{2}+|u(0)|_{2,0, \Omega}^{2}\right],
\end{aligned}
$$

where $\Psi_{16}$ is a positive increasing continuous function of its arguments and $\varepsilon_{i} \in(0,1)(i=1,2,3)$ are sufficiently small constants.

Pr o of. Let $\mathcal{H}$ be the mapping defined by $\mathcal{H}\left(u^{*}\right)=u$, where $u^{*} \in \mathcal{A}_{T}$ is a function appearing on the right-hand sides of (3.41) and $u$ is the solution of (3.41) satisfying estimate (3.44). We shall prove that there exists a fixed point of $\mathcal{H}$. Set $\alpha(t)=\left\|u^{*}\right\|_{\mathcal{A}_{t}}^{2}$ and $\alpha_{0}=\left\|u^{*}(0)\right\|_{0, \Omega}^{2}+\left\|u_{t}^{*}(0)\right\|_{0, \Omega}^{2}$. Assuming $\varepsilon_{4}=t^{a}$ (where $a>0$ is a constant) we have

$$
c\left(t, u^{*}, \varepsilon_{4}\right) \leq \widetilde{c}\left(t^{a} \alpha+\alpha_{0}\right),
$$

where $\widetilde{c}=\widetilde{c}(t)$ is a positive increasing continuous function of $t$. Now, using (3.58) in (3.44) we get

$$
\|u\|_{\mathcal{A}_{t}}^{2} \leq G_{1}(t, d, e)+G_{2}\left(t, e, t^{a} \alpha, \alpha_{0}\right),
$$


where

$$
\begin{aligned}
d= & \|F\|_{1,2,2, \Omega^{t}}^{2}+\left\|F_{t}\right\|_{0, \Omega^{t}}^{2}+\varepsilon_{1}\left\|F_{t t}\right\|_{0, \Omega^{t}}^{2}+\sup _{t}\|F\|_{0, \Omega}^{2} \\
& \quad+\|G\|_{3 / 2,2,2, S^{t}}^{2}+\left\|G_{t}\right\|_{1 / 2,2,2, S^{t}}^{2}+\varepsilon_{1}\left\|G_{t t}\right\|_{0, S^{t}}^{2}+|u(0)|_{2,0, \Omega}^{2}, \\
e= & \frac{1}{\varrho_{*}}+\varrho^{*}+h\left(t, \eta, \varepsilon_{2}\right)+a(w, t)+b\left(t, w, \varepsilon_{3}\right),
\end{aligned}
$$

and $G_{1}$ and $G_{2}$ are positive increasing continuous functions of their arguments. There exist constants $K_{1}, K_{2}>0$ such that

$$
d \leq K_{1}, \quad e \leq K_{2} \quad \text { for } t \leq T .
$$

Let $A>0$ be a constant such that

$$
\alpha(t) \leq A \quad \text { for } t \leq T
$$

and

$$
G_{1}\left(0, K_{1}, K_{2}\right)+G_{2}\left(0, K_{2}, 0, \alpha_{0}\right)<A .
$$

Then by (3.60)-(3.62) there exists $T_{*}>0$ such that

$$
G_{1}(t, d, e)+G_{2}\left(t, e, t^{a} \alpha, \alpha_{0}\right) \leq A \quad \text { for } t \leq T_{*} .
$$

Thus, in view of (3.59) and (3.63) we have proved that if $\alpha(t) \leq A$ then

$$
\|u\|_{\mathcal{A}_{t}}^{2} \leq A \text { for } t \leq T_{*},
$$

where $T_{*}$ is sufficiently small.

Now, it remains to prove that if $u_{1}=\mathcal{H}\left(u_{1}^{*}\right)$ and $u_{2}=\mathcal{H}\left(u_{2}^{*}\right)$, then

$$
\begin{aligned}
\sup _{t}\left\|u_{1}-u_{2}\right\|_{1, \Omega}^{2}+ & \sup _{t}\left\|\left(u_{1}-u_{2}\right)_{, t}\right\|_{0, \Omega}^{2} \\
& +\left\|u_{1}-u_{2}\right\|_{2,2,2, \Omega^{t}}^{2}+\left\|\left(u_{1}-u_{2}\right)_{, t}\right\|_{1,2,2, \Omega^{t}}^{2} \\
< & \sup _{t}\left\|u_{1}^{*}-u_{2}^{*}\right\|_{1, \Omega}^{2}+\sup _{t}\left\|\left(u_{1}^{*}-u_{2}^{*}\right)_{, t}\right\|_{0, \Omega}^{2} \\
& \quad+\left\|u_{1}^{*}-u_{2}^{*}\right\|_{2,2,2, \Omega^{t}}^{2}+\left\|\left(u_{1}^{*}-u_{2}^{*}\right)_{, t}\right\|_{1,2,2, \Omega^{t}}^{2} .
\end{aligned}
$$

Define $U=u_{1}-u_{2}$ and $U^{*}=u_{1}^{*}-u_{2}^{*}$. We see that $U$ is the solution of the problem

$$
\begin{array}{ll}
\eta U_{t}-\operatorname{div} \mathbb{D}(U)=\operatorname{div}_{w} \mathbb{D}_{w}\left(U^{*}\right)-\operatorname{div} \mathbb{D}\left(U^{*}\right) & \text { in } \Omega^{T} \\
\mathbb{D}(U) \cdot \bar{n}_{0}=\mathbb{D}\left(U^{*}\right) \cdot \bar{n}_{0}-\mathbb{D}_{w}\left(U^{*}\right) \cdot \bar{n}_{w} & \text { on } S^{T} \\
\left.U\right|_{t=0}=0 . &
\end{array}
$$

First, we obtain

$$
\begin{aligned}
& \int_{\Omega^{t}} \eta U_{t}^{2} J_{w} d \xi d t^{\prime}+\sup _{t}\|U\|_{1, \Omega}^{2} \\
& \quad \leq \varepsilon\left\|U_{t}\right\|_{1,2,2, \Omega^{t}}^{2}+\delta \Psi_{17}\left(1 / \varrho_{*}, t,\left\|\eta_{t}\right\|_{1,2,2, \Omega^{t}}^{2}\right)\left\|U^{*}\right\|_{1,2,2, \Omega^{t}}^{2}
\end{aligned}
$$


where $\delta$ is the sufficiently small constant from (3.42)-(3.43), $c>0$ is a constant, and $\Psi_{17}$ is a positive increasing continuous function. Next, we have

$$
\begin{aligned}
\sup _{t}\left\|U_{t}\right\|_{0, \Omega}^{2}+\left\|U_{t}\right\|_{1,2,2, \Omega^{t}}^{2} & \\
\leq & \Psi_{18}\left(1 / \varrho_{*}, t,\left\|\eta_{t}\right\|_{1,2,2, \Omega^{t}}^{2}\right)\left[\varepsilon\left\|U^{*}\right\|_{2,2,2, \Omega^{t}}^{2}+\delta\left(1+\sup _{t}\|w\|_{2, \Omega}^{2}\right.\right. \\
& \left.\left.\quad+t \sup _{t}\|w\|_{2, \Omega}^{2}\|w\|_{3,2,2, \Omega^{t}}^{2}\right)\left\|U^{*}\right\|_{1,2,2, \Omega^{t}}^{2}\right],
\end{aligned}
$$

where $\Psi_{18}$ is a positive increasing continuous function and $\varepsilon>0$ is a sufficiently small constant. Adding inequalities (3.66) and (3.67) yields

$$
\begin{aligned}
\sup _{t} \| & U_{t}\left\|_{0, \Omega}^{2}+\right\| U_{t}\left\|_{1,2,2, \Omega^{t}}^{2}+\sup _{t}\right\| U \|_{1, \Omega}^{2} \\
\leq & \Psi_{19}\left(1 / \varrho_{*}, t,\left\|\eta_{t}\right\|_{1,2,2, \Omega^{t}}^{2}\right)\left[\varepsilon\left\|U^{*}\right\|_{2,2,2, \Omega^{t}}^{2}+\delta\left\|U_{t}^{*}\right\|_{1,2,2, \Omega^{t}}^{2}\right. \\
& \left.+\delta\left(1+\sup _{t}\|w\|_{2, \Omega}^{2}+t \sup _{t}\|w\|_{2, \Omega}^{2}\|w\|_{3,2,2, \Omega^{t}}^{2}\right)\left\|U^{*}\right\|_{1,2,2, \Omega^{t}}^{2}\right] .
\end{aligned}
$$

To obtain an estimate for $\|U\|_{2,2,2, \Omega^{t}}^{2}$ we use estimate (A.12) of [6]. We have

$$
\begin{aligned}
\|U\|_{2,2,2, \Omega^{t}}^{2} \leq & c\left(\sup _{t}\|\eta\|_{2, \Omega}^{2}\left\|U_{t}\right\|_{1,2,2, \Omega^{t}}^{2}+\sup _{t}\left\|\eta_{t}\right\|_{1, \Omega}^{2}\|U\|_{1,2,2, \Omega^{t}}^{2}\right. \\
& \left.+\|U\|_{1,2,2, \Omega^{t}}^{2}+\|\widetilde{F}\|_{0, \Omega^{t}}^{2}+\|\widetilde{G}\|_{1 / 2,2,2, S^{t}}^{2}\right)
\end{aligned}
$$

where

$$
\widetilde{F}=\operatorname{div}_{w} \mathbb{D}_{w}\left(U^{*}\right)-\operatorname{div} \mathbb{D}\left(U^{*}\right), \quad \widetilde{G}=\mathbb{D}\left(U^{*}\right) \cdot \bar{n}_{0}-\mathbb{D}_{w}\left(U^{*}\right) \cdot \bar{n}_{w},
$$

so

$$
\begin{aligned}
\|\widetilde{F}\|_{0, \Omega^{t}}^{2} & \leq c \int_{0}^{t}\left(\int_{\Omega}\left|\int_{0}^{t^{\prime}} w_{\xi \xi} d t^{\prime \prime}\right|^{2}\left|U_{\xi}^{*}\right|^{2} d \xi+\int_{\Omega}\left|\xi_{x}-I\right|^{2}\left|U_{\xi \xi}^{*}\right|^{2} d \xi\right) d t^{\prime} \\
& \leq c t\|w\|_{3,2,2, \Omega^{t}}^{2}\left\|U^{*}\right\|_{2,2,2, \Omega^{t}}^{2}+c \delta^{2}\left\|U^{*}\right\|_{2,2,2, \Omega^{t}}^{2}
\end{aligned}
$$

and

$$
\|\widetilde{G}\|_{1 / 2,2,2, S^{t}}^{2} \leq c\left\|U^{*}\right\|_{2,2,2, \Omega^{t}}^{2}\left(t\|w\|_{3,2,2, \Omega^{t}}^{2}+t^{2}\|w\|_{3,2,2, \Omega^{t}}^{4}+\delta^{2}\right) .
$$

By (3.70)-(3.71) we get

$$
\begin{aligned}
& \quad \sup _{t}\|U\|_{1, \Omega}^{2}+\sup _{t}\left\|U_{t}\right\|_{0, \Omega}^{2}+\|U\|_{2,2,2, \Omega^{t}}^{2}+\left\|U_{t}\right\|_{1,2,2, \Omega^{t}}^{2} \\
& \leq \Psi_{20}\left(1 / \varrho_{*}, t, \sup _{t}\left\|\eta_{t}\right\|_{1, \Omega}^{2}, \sup _{t}\|\eta\|_{2, \Omega}^{2}\right) \\
& \times\left[\delta\left(1+\sup _{t}\|w\|_{2, \Omega}^{2}+t \sup _{t}\|w\|_{2, \Omega}^{2}\|w\|_{3,2,2, \Omega^{t}}^{2}\right)\left\|U^{*}\right\|_{1,2,2, \Omega^{t}}^{2}\right. \\
& \left.+\left(\varepsilon+t\|w\|_{3,2,2, \Omega^{t}}^{2}+t^{2}\|w\|_{3,2,2, \Omega^{t}}^{4}+\delta^{2}\right)\left\|U^{*}\right\|_{2,2,2, \Omega^{t}}^{2}+\delta\left\|U_{t}^{*}\right\|_{1,2,2, \Omega^{t}}^{2}\right] .
\end{aligned}
$$


For $\varepsilon, \delta$ and $T_{*}$ sufficiently small inequality (3.72) gives

$$
\begin{aligned}
\sup _{t}\|U\|_{1, \Omega}^{2}+\sup _{t}\left\|U_{t}\right\|_{0, \Omega}^{2}+\|U\|_{2,2,2, \Omega^{t}}^{2}+\left\|U_{t}\right\|_{1,2,2, \Omega^{t}}^{2} \\
<\left\|U^{*}\right\|_{2,2,2, \Omega^{t}}^{2}+\left\|U_{t}^{*}\right\|_{1,2,2, \Omega^{t}}^{2} \quad \text { for } t \leq T_{*} .
\end{aligned}
$$

Since (3.62) implies (3.64) for $t \leq T_{*}$ and (3.73) holds, by the SchauderTikhonov fixed point theorem we obtain the existence of a solution $u \in \mathcal{A}_{T}$ of problem (3.40). The uniqueness of the solution follows from estimate (3.57), so to complete the proof it remains to show this estimate. Since $u$ is a fixed point of $\mathcal{H}$ by Lemma 3.4 it satisfies

$$
\begin{aligned}
\|u\|_{\mathcal{A}_{t}}^{2} \leq & \Psi_{7}\left(1 / \varrho_{*}, \varrho^{*}, t, h\left(t, \eta, \varepsilon_{2}\right)\right)\left[\|F\|_{1,2,2, \Omega^{t}}^{2}+\left\|F_{t}\right\|_{0, \Omega^{t}}^{2}\right. \\
& +\varepsilon_{1}\left\|F_{t t}\right\|_{0, \Omega^{t}}^{2}+\sup _{t}\|F\|_{0, \Omega}^{2} \\
& +\|G\|_{3 / 2,2,2, S^{t}}^{2}+\left\|G_{t}\right\|_{1 / 2,2,2, S^{t}}^{2}+\varepsilon_{1}\left\|G_{t t}\right\|_{0, S^{t}}^{2} \\
& \left.+|u(0)|_{2,0, \Omega}^{2}+c\left(t, u, \varepsilon_{4}\right) \Psi_{8}\left(a(w, t), b\left(t, w, \varepsilon_{3}\right)\right)\right] .
\end{aligned}
$$

Moreover, in [6] (see Lemma 3.7) the following estimate is proved:

$$
\begin{aligned}
\|u\|_{1, \Omega}^{2}+\left\|u_{t}\right\|_{0, \Omega}^{2} & +\|u\|_{1,2,2, \Omega^{t}}^{2}+\left\|u_{t}\right\|_{1,2,2, \Omega^{t}}^{2} \\
\leq & \Psi_{21}\left(t, 1 / \varrho_{*}, a(w, t), b\left(t, w, \varepsilon_{3}\right),\left\|\eta_{t}\right\|_{1,2,2, \Omega^{t}}^{2}\right) \\
& \times\left[\int_{\Omega} \varrho_{0} v_{0}^{2} d \xi+\int_{\Omega} \varrho_{0} u_{t}^{2}(0) d \xi+\left\|v_{0}\right\|_{1, \Omega}^{2}+\left\|F_{t}\right\|_{0, \Omega}^{2}\right. \\
& \left.+\left\|G_{t}\right\|_{0, S^{t}}^{2}+\sup _{t}\|F\|_{0, \Omega}+\sup _{t}\|G\|_{0, S}^{2}\right] .
\end{aligned}
$$

Now, taking into account notation (3.47) and inequalities (3.74)-(3.75), for $t \leq T_{*}$ we get estimate $(3.57)$ if $\varepsilon_{4} \Psi_{7}\left(a(w, t), b\left(t, w, \varepsilon_{3}\right)\right)$ and $T_{*}$ are sufficiently small. This completes the proof of the lemma.

Next, consider the problem

$$
\begin{aligned}
& \eta c_{v}(\eta, \beta) \gamma_{t}-\kappa \nabla_{w}^{2} \gamma=K+\frac{\mu}{2} \sum_{i, j=1}^{3}\left(\xi_{x_{i}} \cdot \nabla_{\xi} w_{j}+\xi_{x_{j}} \cdot \nabla_{\xi} w_{i}\right)^{2} \\
& +(\nu-\mu)\left(\nabla_{w} \cdot w\right)^{2} \quad \text { in } \Omega^{T}, \\
& \bar{n}_{w} \cdot \nabla_{w} \gamma=\bar{\gamma} \quad \text { on } S^{T}, \\
& \left.\gamma\right|_{t=0}=\theta_{0} \quad \text { in } \Omega,
\end{aligned}
$$

where $\xi_{x}=\xi_{x}(w)$ is the inverse matrix to $x_{\xi}=I+\int_{0}^{t} w_{\xi}\left(\xi, t^{\prime}\right) d t^{\prime}$. The following lemma holds.

Lemma 3.6. Let $\eta>0, \eta \in C\left(0, T ; H^{2}(\Omega)\right), \eta_{t} \in C\left(0, T ; H^{1}(\Omega)\right)$, $\eta_{t t} \in L_{2}\left(\Omega^{T}\right), w \in \mathcal{A}_{T}, \beta \in \mathcal{A}_{T}$ (where $\mathcal{A}_{T}$ is given by (2.1)), $\beta>0$, 
$0<\sigma_{*} \leq \eta c_{v}(\eta, \beta) \leq \sigma^{*}\left(\sigma_{*}\right.$ and $\sigma^{*}$ are constants $), K \in H^{2}\left(0, T ; L_{2}(\Omega)\right) \cap$ $L_{2}\left(0, T ; H^{1}(\Omega)\right), \bar{\gamma} \in L_{2}\left(0, T ; H^{3 / 2}(S)\right), \bar{\gamma}_{t} \in L_{2}\left(0, T ; H^{1 / 2}(S)\right), \bar{\gamma}_{t t} \in$ $L_{2}\left(S^{T}\right), S \in H^{5 / 2}, c_{v} \in C^{2}\left(\mathbb{R}_{+}^{2}\right), c_{v}>0, \theta_{0} \in H^{2}(\Omega), \gamma_{t}(0) \in H^{1}(\Omega)$, $\gamma_{t t}(0) \in L_{2}(\Omega)$, (where $\gamma_{t}(0)$ and $\gamma_{t t}(0)$ are calculated from (3.76)). Moreover, let assumptions (3.42)-(3.43) and the following compatibility condition be satisfied:

$$
\bar{n}_{0} \cdot \nabla_{\xi} \theta_{0}=\bar{\theta}(\xi, 0) \quad \text { on } S .
$$

Then there exists $T_{*}>0$ (depending on the arguments of $\Psi_{22}$ and on the expression in square brackets on the right-hand side of (3.77)) such that for $T \leq T_{*}$ there exists a unique solution $\gamma \in \mathcal{A}_{T}$ of problem (3.76) satisfying, for $t \leq T$,

$$
\begin{aligned}
\|\gamma\|_{\mathcal{A}_{t}}^{2} \\
\leq \Psi_{22}\left(1 / \sigma_{*}, \sigma^{*}, t, d_{1}(t, \eta), d_{2}\left(t, \beta, \varepsilon_{5}\right), h\left(t, \eta, \varepsilon_{6}\right), a(w, t), b\left(t, w, \varepsilon_{3}\right)\right) \\
\quad \times\left[\|K\|_{1,2,2, \Omega^{t}}^{2}+\left\|K_{t}\right\|_{0, \Omega^{t}}^{2}+\varepsilon_{1}\left\|K_{t t}\right\|_{0, \Omega^{t}}^{2}+\sup _{t}\|K\|_{0, \Omega}^{2}\right. \\
\quad+\|\bar{\gamma}\|_{3 / 2,2,2, S^{t}}^{2}+\left\|\bar{\gamma}_{t}\right\|_{1 / 2,2,2, S^{t}}^{2}+\varepsilon_{1}\left\|\bar{\gamma}_{t t}\right\|_{0, S^{t}}^{2}+\sup _{t}\|\bar{\gamma}\|_{0, S}^{2} \\
\left.\quad+\mathbf{l} \gamma(0) \mathbf{I}_{2,0, \Omega}^{2}+b\left(t, w, \varepsilon_{3}\right) \Psi_{23}\left(a(w, t), b\left(t, w, \varepsilon_{3}\right)\right)\right],
\end{aligned}
$$

where $\Psi_{22}, \Psi_{23}$ are positive increasing continuous functions of their arguments, $\varepsilon_{i}(i=3,5,6)$ are sufficiently small positive constants; $a, b, h$ are given by (3.45), (3.46) and (3.16), respectively, and

$$
\begin{aligned}
d_{1}\left(t, \beta, \varepsilon_{5}\right) & =\left(\varepsilon_{5}+t^{a_{2}}\right)\left(\sup _{t}\|\beta\|_{2, \Omega}^{2}+\sup _{t}\left\|\beta_{t}\right\|_{1, \Omega}^{2}+\sup _{t}\left\|\beta_{t t}\right\|_{0, \Omega}^{2}\right), \\
d_{2}(t, \eta) & =t^{a_{3}}\left(\sup _{t}\|\eta\|_{2, \Omega}^{2}+\sup _{t}\left\|\eta_{t}\right\|_{1, \Omega}^{2}+\sup _{t}\left\|\eta_{t t}\right\|_{0, \Omega}^{2}\right)
\end{aligned}
$$

$\left(a_{2}, a_{3}>0\right.$ are constants $)$.

Proof. We prove the lemma by using the methods of Lemma 3.5, Lemmas 3.2-3.4 and Lemma 2.2. In particular, setting $\sigma=\eta c_{v}(\eta, \beta)$ by Lemma 2.2 we get

$$
h\left(t, \sigma, \varepsilon_{2}\right) \leq \Psi_{24}\left(d_{1}(t, \eta), d_{2}\left(t, \beta, \varepsilon_{5}\right), h\left(t, \eta, \varepsilon_{6}\right)\right)
$$

(this is used in the proof of estimate (3.77)), where $\Psi_{24}$ is a positive increasing continuous function of its arguments.

4. Existence of solutions of problem (1.1). In order to prove the existence of a solution of problem (1.1) we apply the method of successive approximations. We consider the problems 


$$
\begin{aligned}
& \eta_{m} \partial_{t} u_{m+1}-\mu \nabla_{u_{m}}^{2} u_{m+1}-\nu \nabla_{u_{m}} \nabla_{u_{m}} \cdot u_{m+1} \\
& =-\nabla_{u_{m}} p\left(\eta_{m}, \gamma_{m}\right)+\eta_{m} g_{m} \\
& \eta_{m} c_{v}\left(\eta_{m}, \gamma_{m}\right) \partial_{t} \gamma_{m+1}-\kappa \nabla_{u_{m}}^{2} \gamma_{m+1} \\
& =-\gamma_{m} p_{\gamma}\left(\eta_{m}, \gamma_{m}\right) \nabla_{u_{m}} \cdot u_{m} \\
& +\frac{\mu}{2} \sum_{i, j=1}^{3}\left(\xi_{x_{i}} \cdot \nabla_{\xi} u_{m j}+\xi_{x_{j}} \cdot \nabla_{\xi} u_{m i}\right)^{2} \\
& +(\nu-\mu)\left(\nabla_{u_{m}} \cdot u_{m}\right)^{2}+\eta_{m} k_{m} \quad \text { in } \Omega^{T}, \\
& \eta_{m t}+\eta_{m} \nabla_{u_{m}} \cdot u_{m}=0 \quad \text { in } \Omega^{T} \text {, } \\
& \mathbb{D}_{u_{m}}\left(u_{m+1}\right) \cdot \bar{n}_{u_{m}}=\left[p\left(\eta_{m}, \gamma_{m}\right)-p_{0}\right] \bar{n}_{u_{m}} \quad \text { on } S^{T} \text {, } \\
& \bar{n}_{u_{m}} \cdot \nabla_{u_{m}} \gamma_{m+1}=\bar{\gamma}_{m} \quad \text { on } S^{T} \text {, } \\
& \left.u_{m+1}\right|_{t=0}=v_{0},\left.\quad \gamma_{m+1}\right|_{t=0}=\theta_{0},\left.\quad \eta_{m}\right|_{t=0}=\varrho_{0} \quad \text { in } \Omega,
\end{aligned}
$$

where $\xi_{x}$ is the inverse matrix to $x_{\xi}=I+\int_{0}^{t} u_{m \xi}\left(\xi, t^{\prime}\right) d t^{\prime}$ and

$$
\begin{aligned}
& g_{m}(\xi, t)=f\left(X_{u_{m}}(\xi, t), t\right)=f\left(\xi+\int_{0}^{t} u_{m}\left(\xi, t^{\prime}\right) d t^{\prime}, t\right), \\
& k_{m}(\xi, t)=r\left(X_{u_{m}}(\xi, t), t\right)=r\left(\xi+\int_{0}^{t} u_{m}\left(\xi, t^{\prime}\right) d t^{\prime}, t\right), \\
& \bar{\gamma}_{m}(\xi, t)=\bar{\theta}\left(X_{u_{m}}(\xi, t), t\right)=\bar{\theta}\left(\xi+\int_{0}^{t} u_{m}\left(\xi, t^{\prime}\right) d t^{\prime}, t\right) .
\end{aligned}
$$

For $u_{0}$ we take a function which is a solution of the problem

$$
\begin{array}{ll}
u_{0 t}-\operatorname{div} \mathbb{D}\left(u_{0}\right)=0 & \text { in } \Omega^{T}, \\
\mathbb{D}\left(u_{0}\right) \cdot \bar{n}_{0}=\left(p\left(\varrho_{0}, \theta_{0}\right)-p_{0}\right) \bar{n}_{0} & \text { on } S^{T}, \\
\left.u_{0}\right|_{t=0}=v_{0} & \text { in } \Omega .
\end{array}
$$

By the methods of Lemmas 3.2-3.3 one can prove the following estimate:

$$
\left\|u_{0}\right\|_{\mathcal{A}_{t}}^{2} \leq C_{1}(t)\left[\left\|\left[p\left(\varrho_{0}, \theta_{0}\right)-p_{0}\right] \bar{n}_{0}\right\|_{3 / 2, S}^{2}+\mid u_{0}(0) \mathbf{|}_{2,0, \Omega}^{2}\right] \equiv F_{1}(t),
$$

where $C_{1}$ is a positive increasing continuous function of $t$.

Similarly, for $\gamma_{0}$ we take a function which is a solution of the problem

$$
\begin{array}{ll}
\gamma_{0 t}-\kappa \nabla_{\xi}^{2} \gamma_{0}=0 & \text { in } \Omega^{T}, \\
\bar{n}_{0} \cdot \nabla_{\xi} \gamma_{0}=\bar{\gamma}_{0} & \text { on } S^{T}, \\
\left.\gamma_{0}\right|_{t=0}=\theta_{0} & \text { in } \Omega,
\end{array}
$$

where $\bar{\gamma}_{0}(\xi, t)=\bar{\theta}\left(X_{u_{0}}(\xi, t), t\right)=\bar{\theta}\left(\xi+\int_{0}^{t} u_{0}\left(\xi, t^{\prime}\right) d t^{\prime}, t\right)$. Assume that $\bar{\theta} \in$ $L_{2}\left(0, T ; H_{\mathrm{loc}}^{3}\left(\mathbb{R}^{3}\right)\right) \cap C\left(0, T ; H_{\mathrm{loc}}^{2}\left(\mathbb{R}^{3}\right)\right), \bar{\theta}_{t} \in L_{2}\left(0, T ; H_{\mathrm{loc}}^{2}\left(\mathbb{R}^{3}\right)\right), \bar{\theta}_{t t} \in L_{2}(0, T ;$ 
$\left.H_{\text {loc }}^{1}\left(\mathbb{R}^{3}\right)\right)$. By the methods of Lemmas 3.2-3.4 we get the estimate

$$
\begin{aligned}
\left\|\gamma_{0}\right\|_{\mathcal{A}_{t}}^{2} \leq & C_{2}(t)\left[\left\|\bar{\gamma}_{0}\right\|_{3 / 2,2,2, S^{t}}^{2}+\left\|\bar{\gamma}_{0 t}\right\|_{1 / 2,2,2, S^{t}}^{2}\right. \\
& \left.+\varepsilon_{1}\left\|\bar{\gamma}_{0 t t}\right\|_{0, S^{t}}^{2}+\mathbf{|} \gamma_{0}(0) \mathbf{I}_{2,0, \Omega}^{2}\right]
\end{aligned}
$$

where $C_{2}$ is a positive increasing continuous function of $t$.

Let $A_{1}>0$ be a constant such that

$$
F_{1}(t) \leq A_{1} \quad \text { for } t \leq T .
$$

Define

$$
\Omega_{0 t}=\left\{\xi+\int_{0}^{t} u_{0}\left(\xi, t^{\prime}\right) d t^{\prime}: \xi \in \Omega\right\} .
$$

If $x=\xi+\int_{0}^{t} u_{0}\left(\xi, t^{\prime}\right) d t^{\prime}$, then by (4.6) we have

$$
\operatorname{det}\left\{x_{\xi}\right\} \geq 1-3 t^{1 / 2} A_{1}^{1 / 2}-6 t A_{1}-6 t^{3 / 2} A_{1}^{3 / 2} .
$$

Let $T_{0}>0$ be so small that

$$
1-3 T_{0}^{1 / 2} A_{1}^{1 / 2}-6 T_{0} A_{1}-6 T_{0}^{3 / 2} A_{1}^{3 / 2}>0 .
$$

Then if $T \leq T_{0}$ estimates (4.3), (4.5), (4.7) and (4.8) imply

$$
\begin{array}{r}
\Omega_{0 t} \subset D_{0}=\left\{x=\left(x_{1}, x_{2}, x_{3}\right): \xi_{i}-T_{0}^{1 / 2} A_{1}^{1 / 2} \leq x_{i} \leq \xi_{i}+T_{0}^{1 / 2} A_{1}^{1 / 2},\right. \\
\left.i=1,2,3, \xi=\left(\xi_{1}, \xi_{2}, \xi_{3}\right) \in \Omega\right\} \quad \text { for } t \leq T
\end{array}
$$

and

$$
\begin{aligned}
& \left\|\bar{\gamma}_{0}\right\|_{3 / 2,2,2, S^{t}}^{2}+\left\|\bar{\gamma}_{0 t}\right\|_{1 / 2,2,2, S^{t}}^{2}+\varepsilon_{1}\left\|\bar{\gamma}_{0 t t}\right\|_{0, S^{t}}^{2} \\
& \quad \leq \Psi_{25}\left(\left\|f_{1}\right\|_{C\left(\widetilde{D}_{0}\right)}, A_{1}, t\right)\left(|\nabla \bar{\theta}|_{2,0, D_{0}^{t}}^{2}+\sup _{t}\|\bar{\theta}\|_{2, D_{0}}^{2}\right) \quad \text { for } t \leq T,
\end{aligned}
$$

where $\Psi_{25}$ is a positive increasing continuous function of its arguments, $\operatorname{det} \xi_{x}=f_{1}\left(I+\int_{0}^{t} u_{0 \xi} d t^{\prime}\right), f_{1}$ is a continuous function, $\widetilde{D}_{0}=\left\{z=\left(z_{i j}\right)_{i, j=1,2,3}\right.$ : $\left.\delta_{i j}-T_{0}^{1 / 2} A_{1}^{1 / 2} \leq z_{i j} \leq \delta_{i j}+T_{0}^{1 / 2} A_{1}^{1 / 2}, i=1,2,3\right\}, \delta_{i j}$ is the Kronecker delta, and $D_{0}^{t}=D_{0} \times(0, t)$. By (4.5) and (4.9) we have

$$
\begin{aligned}
\left\|\gamma_{0}\right\|_{\mathcal{A}_{t}}^{2} \leq & \Psi_{26}\left(\left\|f_{1}\right\|_{C\left(\widetilde{D}_{0}\right)}, A_{1}, t\right) \\
& \times\left[|\nabla \bar{\theta}|_{2,0, D_{0}^{t}}^{2}+\sup _{t}\|\bar{\theta}\|_{2, D_{0}}^{2}+\left|\mathbf{l} \gamma_{0}(0)\right|_{2,0, \Omega}^{2}\right] \equiv F_{2}(t) .
\end{aligned}
$$

Finally, $\eta_{0}$ is a solution of the problem

$$
\begin{array}{ll}
\eta_{0 t}+\eta_{0} \nabla_{u_{0}} \cdot u_{0}=0 & \text { in } \Omega^{T}, \\
\left.\eta_{0}\right|_{t=0}=\varrho_{0} & \text { in } \Omega .
\end{array}
$$

Now, we prove the following lemma.

LEMmA 4.1. Assume that $v_{0} \in H^{2}(\Omega), \varrho_{0} \in H^{2}(\Omega), \theta_{0} \in H^{2}(\Omega)$ and there exist positive constants $\varrho_{*}, \varrho^{*}, \theta_{*}, \theta^{*}\left(\varrho_{*}<\varrho^{*}, \theta_{*}<\theta^{*}\right)$ such that

$$
\varrho_{*}<\varrho_{0}<\varrho^{*}, \quad \theta_{*}<\theta_{0}<\theta^{*} .
$$


Moreover, assume that $u_{t}(0), \gamma_{t}(0) \in H^{1}(\Omega), u_{t t}(0), \gamma_{t t}(0) \in L_{2}(\Omega)$ (where $u_{t}(0), u_{t t}(0), \gamma_{t}(0), \gamma_{t t}(0)$ are calculated from system $\left.(1.4)\right), u_{0 t}(0), \gamma_{0 t}(0) \in$ $H^{1}(\Omega), u_{0 t t}(0), \gamma_{0 t t}(0) \in L_{2}(\Omega)$, (where $u_{0 t}(0), u_{0 t t}(0), \gamma_{0 t}(0), \gamma_{0 t t}(0)$ are calculated from systems (4.2) and (4.4)). Let $f, r \in L_{2}\left(0, T ; H_{\text {loc }}^{2}\left(\mathbb{R}^{3}\right)\right), f_{t}, r_{t}$ $\in L_{2}\left(0, T ; H_{\mathrm{loc}}^{1}\left(\mathbb{R}^{3}\right)\right), f_{t t}, r_{t t} \in L_{2}\left(0, T ; L_{2}\right.$ loc $\left.\left(\mathbb{R}^{3}\right)\right), \bar{\theta} \in L_{2}\left(0, T ; H_{\mathrm{loc}}^{2}\left(\mathbb{R}^{3}\right)\right) \cap$ $C\left(0, T ; H_{\mathrm{loc}}^{2}\left(\mathbb{R}^{3}\right)\right), \bar{\theta}_{t} \in L_{2}\left(0, T ; H_{\mathrm{loc}}^{2}\left(\mathbb{R}^{3}\right)\right), \bar{\theta}_{t t} \in L_{2}\left(0, T ; H_{\mathrm{loc}}^{1}\left(\mathbb{R}^{3}\right)\right), S \in$ $H^{5 / 2}, p \in C^{3}\left(\mathbb{R}_{+}^{2}\right), c_{v} \in C^{2}\left(\mathbb{R}_{+}^{2}\right), c_{v}>0$ and assume the following compatibility conditions are satisfied:

$$
\mathbb{D}\left(v_{0}\right) \cdot \bar{n}_{0}=\left(p\left(\varrho_{0}, \theta_{0}\right)-p_{0}\right) \bar{n}_{0} \quad \text { on } S
$$

and

Define

$$
\bar{n}_{0} \cdot \nabla_{\xi} \theta_{0}=\bar{\theta}(\xi, 0) \quad \text { on } S .
$$

Let $A>0$ be a constant satisfying (4.31) and

$$
\alpha_{m}(t)=\left\|u_{m}\right\|_{\mathcal{A}_{t}}^{2}+\left\|\gamma_{m}\right\|_{\mathcal{A}_{t}}^{2} .
$$

$$
F_{1}(t)+F_{2}(t) \leq A \quad \text { for } t \leq T_{0}
$$

(where $F_{1}(t)$ and $F_{2}(t)$ are defined in (4.3) and (4.10), and $T_{0}$ is given by (4.8)). Then there exists $0<T_{*} \leq T_{0}$ such that if $T \leq T_{*}$ we have

$$
\alpha_{m}(t) \leq A \quad \text { for } t \leq T \text { and } m=0,1,2, \ldots
$$

Pro of. By (4.12) we have

$$
\alpha_{0}(t) \leq A \quad \text { for } t \leq T_{0} .
$$

Now, we assume that for some $m>0$ there exists $0<T_{*} \leq T_{0}$ such that if $T \leq T_{*}$ then

$$
\alpha_{m}(t) \leq A \quad \text { for } t \leq T .
$$

We shall prove that $\alpha_{m+1}(t) \leq A$ for $t \leq T$. In order to do this define

(4.16) $\widetilde{M}=\bar{\gamma}_{m}+\left[p\left(\eta_{m}, \gamma_{m}\right)-p_{0}\right] \bar{n}_{u_{m}}$

and introduce the set

$$
\Omega_{m t}=\left\{\xi+\int_{0}^{t} u_{m}\left(\xi, t^{\prime}\right) d t^{\prime}: \xi \in \Omega\right\} .
$$

If $x=\xi+\int_{0}^{t} u_{m}\left(\xi, t^{\prime}\right) d t^{\prime}$, then by (4.14) we have

$$
\operatorname{det}\left\{x_{\xi}\right\} \geq 1-3 t^{1 / 2} A^{1 / 2}-6 t A-6 t^{3 / 2} A^{3 / 2} .
$$

Let $T_{*}>0$ be so small that

$$
1-3 T_{*}^{1 / 2} A^{1 / 2}-6 T_{*} A-6 T_{*}^{3 / 2} A^{3 / 2}>0 .
$$


Then for $T \leq T_{*}$ estimates (4.14), (4.17), (4.18) imply

$$
\begin{array}{r}
\Omega_{m t} \subset D_{*}=\left\{x=\left(x_{1}, x_{2}, x_{3}\right): \xi_{i}-T_{*}^{1 / 2} A^{1 / 2} \leq x_{i} \leq \xi_{i}+T_{*}^{1 / 2} A^{1 / 2},\right. \\
\left.i=1,2,3, \xi=\left(\xi_{1}, \xi_{2}, \xi_{3}\right) \in \Omega\right\} \quad \text { for } t \leq T
\end{array}
$$

and

$$
\begin{aligned}
& \left|g_{m}\right|_{2,0, \Omega^{t}}^{2}+\sup _{t}\left\|g_{m}\right\|_{0, \Omega}^{2}+\left|k_{m}\right|_{2,0, \Omega^{t}}^{2}+\sup _{t}\left\|k_{m}\right\|_{0, \Omega}^{2} \\
& \leq \Psi_{27}\left(\left\|f_{1}\right\|_{C\left(\widetilde{D}_{*}\right)},|f|_{2,0, D_{*}^{T *}}^{2}, \sup _{t}\|f\|_{0, D_{*}}^{2},|r|_{2,0, D_{*}^{T *}}^{2},\right. \\
& \left.\sup _{t}\|r\|_{0, D_{*}}^{2}, a\left(u_{m}, t\right), b\left(t, u_{m}, \varepsilon_{3}\right)\right),
\end{aligned}
$$

$$
\left\|\bar{\gamma}_{m}\right\|_{3 / 2,2,2, S^{t}}^{2}+\left\|\bar{\gamma}_{m t}\right\|_{1 / 2,2,2, S^{t}}^{2}+\left\|\bar{\gamma}_{m t t}\right\|_{0, S^{t}}^{2}+\sup _{t}\left\|\bar{\gamma}_{m}\right\|_{0, S}^{2}
$$

$\leq \Psi_{28}\left(\left\|f_{1}\right\|_{C\left(\widetilde{D}_{*}\right)},|\nabla \bar{\theta}|_{2,0, D_{*}^{T *}}^{2}, \sup _{t}\|\bar{\theta}\|_{2, D_{*}}^{2}, a\left(u_{m}, t\right), b\left(t, u_{m}, \varepsilon_{3}\right)\right) \quad$ for $t \leq T$,

where $D_{*}^{T_{*}}=D_{*} \times\left(0, T_{*}\right), f_{1}=\operatorname{det} \xi_{x}$ is a continuous function of $I+$ $\int_{0}^{t} u_{m \xi} d t^{\prime}, \widetilde{D}_{*}=\left\{z=\left(z_{i j}\right)_{i, j=1,2,3}: \delta_{i j}-T_{*}^{1 / 2} A^{1 / 2} \leq z_{i j} \leq \delta_{i j}+T_{*}^{1 / 2} A^{1 / 2}\right.$, $i, j=1,2,3\}, \Psi_{27}$ and $\Psi_{28}$ are positive increasing continuous functions of their arguments.

By (4.15), (4.16), (4.19) and (4.20) we have

$$
\begin{aligned}
& \|\widetilde{L}\|_{1,2,2, \Omega^{t}}^{2}+\left\|\widetilde{L}_{t}\right\|_{0, \Omega^{t}}^{2}+\varepsilon_{1}\left\|\widetilde{L}_{t t}\right\|_{0, \Omega^{t}}^{2}+\sup _{t}\|\widetilde{L}\|_{0, \Omega}^{2} \\
& \quad+\|\widetilde{M}\|_{3 / 2,2,2, S^{t}}^{2}+\left\|\widetilde{M}_{t}\right\|_{1 / 2,2,2, S^{t}}^{2}+\varepsilon_{1}\left\|\widetilde{M}_{t t}\right\|_{0, S^{t}}^{2}+\sup _{t}\|\widetilde{M}\|_{0, S}^{2} \\
& \leq \Psi_{29}\left(\omega_{*}, e\left(t, \eta_{m}\right), a\left(u_{m}, t\right), b\left(t, u_{m}, \varepsilon_{3}\right), b\left(t, \gamma_{m}, \varepsilon_{8}\right)\right) \\
& \quad+\varepsilon_{1}\left(\left|\eta_{m t}\right|_{2,1, \Omega^{t}}^{2}+\left|\gamma_{m t}\right|_{2,1, \Omega^{t}}^{2}+\left\|\gamma_{m t t}\right\|_{1,2,2}^{2}\right) \\
& \quad \times \Psi_{30}\left(e\left(t, \eta_{m}\right), b\left(t, u_{m}, \varepsilon_{3}\right), b\left(t, \gamma_{m}, \varepsilon_{8}\right)\right),
\end{aligned}
$$

where $\Psi_{29}$ and $\Psi_{30}$ are positive increasing continuous functions, $\varepsilon_{1}>0$ is a sufficiently small constant and

$$
\begin{aligned}
\omega_{*}= & \left\|f_{1}\right\|_{C\left(\widetilde{D}_{*}\right)}+|f|_{2,0, D_{*}^{T *}}^{2}+\sup _{t}\|f\|_{0, D_{*}}^{2}+|r|_{2,0, D_{*}^{T_{*}}}^{2} \\
& +\sup _{t}\|r\|_{0, D_{*}}^{2}+|\nabla \bar{\theta}|_{2,0, D_{*}^{2}}^{2}+\sup _{t}\|\bar{\theta}\|_{2, D_{*}}^{2}, \\
e\left(t, \eta_{m}\right)= & \sup _{t}\left\|\eta_{m t t}\right\|_{0, \Omega}^{2}+\sup _{t}\left\|\eta_{m t}\right\|_{1, \Omega}^{2}+\sup _{t}\left\|\eta_{m}\right\|_{2, \Omega}^{2} .
\end{aligned}
$$

The functions $\Psi_{i}$ in (4.19)-(4.21) also depend on $\|p\|_{C^{3}\left(\bar{V}_{m}\right)}$, where $V_{m} \subset \mathbb{R}_{+}^{3}$ is a bounded domain such that $\left(\eta_{m}(\xi, t), \gamma_{m}(\xi, t)\right) \in V_{m}$ for any $(\xi, t) \in \Omega^{T}$. Assuming that $\varepsilon_{i}=t^{a}(i=1,3,5,7,8)$ (where $a=\min \left(a_{1}, a_{2}, a_{3}, 1\right), a_{i}>0$ $(i=1,2,3)$ are constants from Lemma $2.3,(3.78)$ and (3.79), respectively), using the definitions (3.16), (3.45), (3.46), (3.78), (3.79), Lemma 2.3 and 
the estimates

$$
\begin{aligned}
& \sup _{t}\left\|\eta_{m}\right\|_{0, \Omega}^{2} \leq \sup _{t}\left\|\int_{0}^{t} \eta_{m t} d t^{\prime}\right\|_{0, \Omega}^{2}+\left\|\varrho_{0}\right\|_{0, \Omega}^{2} \leq t \sup _{t}\left\|\eta_{m t}\right\|_{0, \Omega}^{2}+\left\|\varrho_{0}\right\|_{0, \Omega}^{2}, \\
& \sup _{t}\left\|\eta_{m t}\right\|_{0, \Omega}^{2} \leq t \sup _{t}\left\|\eta_{m t t}\right\|_{0, \Omega}^{2}+c\left\|\varrho_{0}\right\|_{1, \Omega}^{2}\left\|v_{0}\right\|_{2, \Omega}^{2}
\end{aligned}
$$

we obtain

$$
\begin{aligned}
d_{1}\left(t, \gamma_{m}, \varepsilon_{5}\right)+d_{2}( & \left.t, \eta_{m}\right)+h\left(t, \eta_{m}, \varepsilon_{7}\right)+a\left(u_{m}, t\right)+b\left(t, u_{m}, \varepsilon_{3}\right) \\
& +b\left(t, \gamma_{m}, \varepsilon_{8}\right)+e\left(t, \eta_{m}\right) \\
& +\varepsilon_{1}\left(\left|\eta_{m t}\right|_{2,1, \Omega^{t}}^{2}+\left|\gamma_{m t}\right|_{2,1, \Omega^{t}}^{2}+\left\|u_{m t t}\right\|_{1,2,2, \Omega^{t}}^{2}\right) \\
\leq & c\left(t^{a} \alpha_{m}+F_{0}\right),
\end{aligned}
$$

where $c>0$ is a constant and

$$
F_{0}=\left\|v_{0}\right\|_{2, \Omega}^{2}+\left\|u_{t}(0)\right\|_{1, \Omega}^{2}+\left\|\theta_{0}\right\|_{2, \Omega}^{2}+\left\|\gamma_{t}(0)\right\|_{1, \Omega}^{2}+\left\|\varrho_{0}\right\|_{2, \Omega}^{2}
$$

$\left(u_{t}(0)\right.$ and $\gamma_{t}(0)$ are calculated from (1.4)).

Now, by (4.14) we have

$$
\begin{aligned}
\theta_{0}-\left(c_{0} t A\right)^{1 / 2} & \leq \theta_{0}-t^{1 / 2}\left(\int_{0}^{t}\left|\partial_{t} \gamma_{m}\left(\xi, t^{\prime}\right)\right|^{2} d t^{\prime}\right)^{1 / 2} \leq \gamma_{m}(t) \\
& \leq \theta_{0}+t^{1 / 2}\left(\int_{0}^{t}\left|\partial_{t} \gamma_{m}\left(\xi, t^{\prime}\right)\right|^{2} d t^{\prime}\right)^{1 / 2} \\
& \leq \theta_{0}+\left(c_{0} t A\right)^{1 / 2} \text { for } t \leq T
\end{aligned}
$$

(where $c_{0}>0$ is a constant from the inequality $|w|_{\infty, \Omega} \leq c_{0}\|w\|_{2, \Omega}^{2}$ ) and

$$
\begin{aligned}
\varrho_{0} \exp \left[-\left(c_{0} t A\right)^{1 / 2}\right] & \leq \varrho_{0} \exp \left[t^{1 / 2}\left(\int_{0}^{t}\left|\nabla_{u_{m}} \cdot u_{m}\right|^{2} d t^{\prime}\right)^{1 / 2}\right] \\
& \leq \eta_{m}(t) \leq \varrho_{0} \exp \left[t^{1 / 2}\left(\int_{0}^{t}\left|\nabla_{u_{m}} \cdot u_{m}\right|^{2} d t^{\prime}\right)^{1 / 2}\right] \\
& \leq \varrho_{0} \exp \left[\left(c_{0} t A\right)^{1 / 2}\right] \quad \text { for } t \leq T .
\end{aligned}
$$

Let $T_{*}$ be so small that (see assumption (4.11))

$$
\theta_{0}-\left(c_{0} T_{*} A\right)^{1 / 2}>\theta_{*}, \quad \theta_{0}+\left(c_{0} T_{*} A\right)^{1 / 2}<\theta^{*}
$$

and

$$
\varrho_{0} \exp \left[-\left(c_{0} T_{*} A\right)^{1 / 2}\right]>\varrho_{*}, \quad \varrho_{0} \exp \left[\left(c_{0} T_{*} A\right)^{1 / 2}\right]<\varrho^{*} .
$$

Then in view of $(4.23)-(4.26)$ we get

$$
\varrho_{*}<\gamma_{m}(t)<\varrho^{*}
$$


and

$$
\theta_{*}<\eta_{m}(t)<\theta^{*} \quad \text { for } t \leq T \leq T_{*} .
$$

Denote by $\sigma_{*}$ a constant satisfying

$$
\varrho_{0} c_{v}\left(\varrho_{0}, \theta_{0}\right) \geq \varrho_{*} \inf _{V} c_{v}(\eta, \gamma)>\sigma_{*}>0,
$$

where $V=\left\{(\eta, \gamma): \varrho_{*}<\eta<\varrho^{*}, \theta_{*}<\gamma<\theta^{*}\right\}$. Then (4.27)-(4.28) yield

$$
\eta_{m} c_{v}\left(\eta_{m}, \gamma_{m}\right)>\sigma_{*} \quad \text { for } t \leq T \text {. }
$$

Now, in view of (4.21), (4.22), (4.27) and (4.29), Lemmas 3.5-3.6 imply that if $T_{*}$ is sufficiently small then for $T \leq T_{*}$ there exists a unique solution $u_{m+1} \in \mathcal{A}_{T}$ of $(4.1)_{1}$ and a unique solution $\gamma_{m+1} \in \mathcal{A}_{T}$ of $(4.1)_{2}$ satisfying

$$
\alpha_{m+1}(t) \leq G\left(t, t^{a} \alpha_{m}(t), \omega_{*}, H_{0}\right) \quad \text { for } t \leq T,
$$

where $G$ is a positive increasing continuous function of its arguments,

$$
H_{0}=\frac{1}{\varrho_{*}}+\frac{1}{\sigma_{*}}+\varrho^{*}+\sigma^{*}+F_{0}+\left\|u_{t t}(0)\right\|_{0, \Omega}^{2}+\left\|\gamma_{t t}(0)\right\|_{0, \Omega}^{2} \leq \widetilde{H}_{0},
$$

and $\widetilde{H}_{0}$ is a constant. Assume that the constant $A$ is so large that

$$
G\left(0,0, \widetilde{H}_{0}\right)<A .
$$

Then by (4.14) and (4.30)-(4.31) if $T_{*}$ is so small that

$$
G\left(T_{*}, T_{*}^{a} A, \widetilde{H}_{0}\right)<A
$$

we obtain $\alpha_{m+1}(t) \leq A$ for $t \leq T \leq T_{*}$. This concludes the proof.

Now, we prove the main result of the paper.

THeOREM 4.2. Let the assumptions of Lemma 4.1 be satisfied. Then there exists $T_{*}>0$ such that for $T \leq T_{*}$ there exists a unique solution $(u, \gamma, \eta)$ of problem (1.4) such that $u, \gamma \in \mathcal{A}_{T}, \eta \in \mathcal{B}_{T}\left(\mathcal{A}_{T}\right.$ and $\mathcal{B}_{T}$ are given by (2.1) and (2.2)) and

$$
\|u\|_{\mathcal{A}_{T}}^{2}+\|\gamma\|_{\mathcal{A}_{T}}^{2} \leq A
$$

and

$$
\|\eta\|_{\mathcal{B}_{T}}^{2} \leq \phi(A),
$$

where $A>0$ is given by (4.12) and (4.31), and $\phi$ is a positive increasing continuous function.

P r o of. In view of Lemma 4.1 it remains to show the convergence of the sequence $\left(u_{m}, \gamma_{m}, \eta_{m}\right)$ and the uniqueness. Consider the following system of problems for the differences $U_{m+1}=u_{m+1}-u_{m}, \Gamma_{m+1}=\gamma_{m+1}-\gamma_{m}$ 
and $H_{m}=\eta_{m}-\eta_{m-1}$ :

$$
\begin{aligned}
& \eta_{m} \partial_{t} U_{m+1}-\mu \nabla_{u_{m}}^{2} U_{m+1}-\nu \nabla_{u_{m}} \nabla_{u_{m}} \cdot U_{m+1}=\sum_{i=1}^{5} F_{i} \equiv \widetilde{F}, \\
& \mathbb{D}_{u_{m}}\left(U_{m+1}\right) \cdot \bar{n}_{u_{m}}=\sum_{i=1}^{3} G_{i} \equiv \widetilde{G} \\
& \left.U_{m+1}\right|_{t=0}=0 \\
& \eta_{m} c_{v}\left(\eta_{m}, \gamma_{m}\right) \partial_{t} \Gamma_{m+1}-\kappa \nabla_{u_{m}}^{2} \Gamma_{m+1}=\sum_{i=1}^{7} I_{i} \equiv \widetilde{I}, \\
& \bar{n}_{u_{m}} \cdot \nabla_{u_{m}} \Gamma_{m+1}=J_{1}+J_{2} \equiv \widetilde{J}, \\
& \left.\Gamma_{m+1}\right|_{t=0}=0 \\
& \partial_{t} H_{m}+H_{m} \operatorname{div}_{u_{m}} u_{m}=-\eta_{m-1}\left(\operatorname{div}_{u_{m}} u_{m}-\operatorname{div}_{u_{m-1}} u_{m-1}\right), \\
& \left.H_{m}\right|_{t=0}=0
\end{aligned}
$$

where $\operatorname{div}_{u_{i}} u_{i}=\nabla_{u_{i}} \cdot u_{i}$ and

$$
\begin{aligned}
F_{1}= & -H_{m} \partial_{t} u_{m}, \\
F_{2}= & -\mu\left(\nabla_{u_{m}}^{2}-\nabla_{u_{m-1}}^{2}\right) u_{m}-\nu\left(\nabla_{u_{m}} \nabla_{u_{m}} \cdot-\nabla_{u_{m-1}} \nabla_{u_{m-1}} \cdot\right) u_{m} \\
F_{3}= & \nabla_{u_{m}} p\left(\eta_{m}, \gamma_{m}\right)-\nabla_{u_{m-1}} p\left(\eta_{m-1}, \gamma_{m-1}\right), \\
F_{4}= & H_{m} g_{m}, \quad F_{5}=\eta_{m-1}\left(g_{m}-g_{m-1}\right) \\
G_{1}= & -\left[\mathbb{D}_{u_{m}}\left(u_{m}\right) \cdot \bar{n}_{u_{m}}-\mathbb{D}_{u_{m-1}}\left(u_{m}\right) \cdot \bar{n}_{u_{m-1}}\right], \\
G_{2}= & p\left(\eta_{m}, \gamma_{m}\right) \bar{n}_{u_{m}}-p\left(\eta_{m-1}, \gamma_{m-1}\right) \bar{n}_{u_{m-1}}, \\
G_{3}= & -p_{0}\left(\bar{n}_{u_{m}}-\bar{n}_{u_{m-1}}\right) \\
I_{1}= & -H_{m} c_{v}\left(\eta_{m}, \gamma_{m}\right) \partial_{t} \gamma_{m}, \\
I_{2}= & \eta_{m-1} \partial_{t} \gamma_{m}\left[c_{v}\left(\eta_{m-1}, \gamma_{m-1}\right)-c_{v}\left(\eta_{m}, \gamma_{m}\right)\right] \\
I_{3}= & \frac{\mu}{2} \sum_{i, j=1}^{3}\left[\left(\xi_{x_{i}} \cdot \nabla_{\xi} u_{m j}+\xi_{x_{j}} \cdot \nabla_{\xi} u_{m i}\right)^{2}\right. \\
& \left.-\left(\xi_{x_{i}} \cdot \nabla_{\xi} u_{m-1, j}+\xi_{x_{j}} \cdot \nabla_{\xi} u_{m-1, i}\right)^{2}\right] \\
I_{4}= & (\nu-\mu)\left[\left(\nabla_{u_{m}} \cdot u_{m}\right)^{2}-\left(\nabla_{u_{m-1}} \cdot u_{m-1}\right)^{2}\right] \\
I_{5}= & -\gamma_{m} p_{r}\left(\eta_{m}, \gamma_{m}\right) \nabla_{u_{m}} \cdot u_{m}+\gamma_{m-1} p_{r}\left(\eta_{m-1}, \gamma_{m-1}\right) \nabla_{u_{m-1}} \cdot u_{m-1}, \\
I_{6}= & H_{m} k_{m}, \quad I_{7}=\eta_{m-1}\left(k_{m}-k_{m-1}\right) \\
J_{1}= & -\left(\bar{n}_{u_{m}} \cdot \nabla_{u_{m}} \gamma_{m}-\bar{n}_{u_{m-1}} \cdot \nabla_{u_{m-1}} \gamma_{m}\right), \\
J_{2}= & \bar{\gamma}_{m}-\bar{\gamma}_{m-1} \cdot
\end{aligned}
$$

Now, multiply $(4.34)_{1}$ by $U_{m+1} J_{u_{m}}$ (where $J_{u_{m}}$ is the Jacobian of the transformation $\left.x=\xi+\int_{0}^{t} u_{m} d t^{\prime}\right)$ and integrate over $\Omega$. Then integration by 
parts using $(4.34)_{2}$ yields

$$
\begin{aligned}
& \quad \frac{1}{2} \int_{\Omega}\left(\eta_{m} \frac{d}{d t} U_{m+1}^{2} J_{u_{m}}+\frac{\mu}{2}\left|\mathbb{S}_{u_{m}}\left(U_{m+1}\right)\right|^{2} J_{u_{m}}\right) d \xi \\
& +(\nu-\mu) \int_{\Omega}\left(\operatorname{div}_{u_{m}} U_{m+1}\right)^{2} J_{u_{m}} d \xi=\int_{\Omega} \widetilde{F} U_{m+1} J_{u_{m}} d \xi+\int_{S} \widetilde{G} U_{m+1} J_{u_{m}} d \xi .
\end{aligned}
$$

We now estimate each terms on the r.h.s. of (4.35). Using (4.13) we get

$$
\begin{aligned}
& \left|\int_{\Omega^{t}} H_{m} u_{m t} U_{m+1} J_{u_{m}} d \xi d t^{\prime}\right| \\
& \leq \varepsilon\left\|U_{m+1}\right\|_{1,2,2, \Omega^{t}}^{2}+c(\varepsilon) \phi(A) \sup _{t}\left\|H_{m}\right\|_{1, \Omega}^{2}
\end{aligned}
$$

(here and in the sequel we denote by $\phi$ different positive continuous functions of $A$ ),

$$
\begin{aligned}
& \left|\int_{\Omega^{t}} F_{2} U_{m+1} J_{u_{m}} d \xi d t^{\prime}\right| \\
& \leq c\left(\int_{\Omega^{t}}\left|\int_{0}^{t^{\prime}} U_{m \xi} d t^{\prime \prime}\right|\left|u_{m \xi \xi}\right|\left|U_{m+1}\right|\left|J_{u_{m}}\right| d \xi d t^{\prime}\right. \\
& +\int_{\Omega^{t}}\left|\int_{0}^{t} U_{m \xi} d t^{\prime \prime}\right|\left|\int_{0}^{t^{\prime}} u_{m \xi \xi} d t^{\prime \prime}\right|\left|u_{m \xi}\right|\left|U_{m+1}\right|\left|J_{u_{m}}\right| d \xi d t^{\prime} \\
& +\int_{\Omega^{t}}\left|\int_{0}^{t^{\prime}} U_{m \xi} d t^{\prime \prime}\right|\left|\int_{0}^{t^{\prime}} u_{m-1 \xi \xi} d t^{\prime \prime}\right|\left|u_{m \xi}\right|\left|U_{m+1}\right|\left|J_{u_{m}}\right| d \xi d t^{\prime} \\
& \left.+\int_{\Omega^{t}}\left|\int_{0}^{t^{\prime}} U_{m \xi \xi} d t^{\prime \prime}\right|\left|u_{m \xi}\right|\left|U_{m+1}\right|\left|J_{u_{m}}\right| d \xi d t^{\prime}\right) \\
& \leq \varepsilon\left\|U_{m+1}\right\|_{1,2,2, \Omega^{t}}^{2}+c(\varepsilon) \phi(A) t\left\|U_{m}\right\|_{2,2,2, \Omega^{t}}^{2}, \\
& \text { (4.38) }\left|\int_{\Omega^{t}} F_{3} U_{m+1} J_{u_{m}} d \xi d t^{\prime}\right| \\
& \leq \varepsilon\left\|U_{m+1}\right\|_{1,2,2, \Omega^{t}}^{2}+c(\varepsilon) \phi(A) t\left(\left\|U_{m}\right\|_{2,2,2, \Omega^{t}}^{2}\right. \\
& \left.+\sup _{t}\left\|H_{m}\right\|_{1, \Omega}^{2}+\sup _{t}\left\|\Gamma_{m}\right\|_{1, \Omega}^{2}\right), \\
& \leq \varepsilon\left\|U_{m+1}\right\|_{1,2,2, \Omega^{t}}^{2}+c(\varepsilon) \phi(A) t \sup _{t}\left\|H_{m}\right\|_{0, \Omega}^{2},
\end{aligned}
$$$$
\text { (4.39) }\left|\int_{\Omega^{t}} F_{4} U_{m+1} J_{u_{m}} d \xi d t^{\prime}\right|
$$ 
$(4.40)$

$$
\begin{gathered}
\text { (4.40) } \quad\left|\int_{\Omega^{t}} F_{5} U_{m+1} J_{u_{m}} d \xi d t^{\prime}\right| \\
\leq \varepsilon\left\|U_{m+1}\right\|_{1,2,2, \Omega^{t}}^{2}+c(\varepsilon) \phi(A) t\left\|U_{m}\right\|_{1,2,2, \Omega^{t}}^{2}, \\
(4.41) \quad\left|\int_{S^{t}}\left(G_{1}+G_{3}\right) U_{m+1} J_{u_{m}} d \xi_{s} d t^{\prime}\right| \\
\leq \varepsilon\left\|U_{m+1}\right\|_{1,2,2, \Omega^{t}}^{2}+c(\varepsilon) \phi(A) t\left\|U_{m}\right\|_{2,2,2, \Omega^{t}}^{2}, \\
(4.42) \quad\left|\int_{S^{t}} G_{2} U_{m+1} J_{u_{m}} d \xi_{s} d t^{\prime}\right| \\
\leq \varepsilon\left\|U_{m+1}\right\|_{1,2,2, \Omega^{t}}^{2}+c(\varepsilon) \phi(A) t\left(\left\|U_{m}\right\|_{2,2,2, \Omega^{t}}^{2}\right. \\
\left.\quad+\sup _{t}\left\|H_{m}\right\|_{1, \Omega}^{2}+\sup _{t}\left\|\Gamma_{m}\right\|_{1, \Omega}^{2}\right) .
\end{gathered}
$$

From (4.35)-(4.42) and the Korn inequality we obtain, for sufficiently small $\varepsilon$,

$$
\begin{aligned}
\left\|U_{m+1}\right\|_{0, \Omega}^{2}+\left\|U_{m+1}\right\|_{1,2,2, \Omega^{t}}^{2} & \leq t \phi(A)\left(\left\|U_{m}\right\|_{2,2,2, \Omega^{t}}^{2}+\sup _{t}\left\|H_{m}\right\|_{1, \Omega}^{2}+\sup _{t}\left\|\Gamma_{m}\right\|_{1, \Omega}^{2}\right) .
\end{aligned}
$$

Multiplying (4.34) $)_{1}$ by $U_{m+1, t} J_{u_{m}}$, integrating over $\Omega$ and by parts we get

$$
\begin{aligned}
\int_{\Omega} \eta_{m}\left|U_{m+1, t}\right|^{2} J_{u_{m}} d \xi+ & \frac{\mu}{2} \int_{\Omega} \mathbb{S}_{u_{m}}\left(U_{m+1}\right) \mathbb{S}_{u_{m}}\left(U_{m+1, t}\right) J_{u_{m}} d \xi \\
& +(\nu-\mu) \int_{\Omega} \operatorname{div}_{u_{m}} U_{m+1} \operatorname{div}_{u_{m}} U_{m+1, t} J_{u_{m}} d \xi \\
& =\int_{S} \widetilde{G} U_{m+1, t} J_{u_{m}} d \xi_{s}+\int_{\Omega} \widetilde{F} U_{m+1, t} J_{u_{m}} d \xi .
\end{aligned}
$$

Hence, by the same calculations as in [6] (see (3.51)-(3.52) of [6]) we have

$$
\begin{aligned}
\left\|U_{m+1, t}\right\|_{0, \Omega^{t}}^{2}+\left\|U_{m+1}\right\|_{1, \Omega}^{2} & \\
\leq & \varepsilon_{2} \sup _{t}\|\widetilde{G}\|_{0, S}^{2}+c\left(\varepsilon_{2}\right)\left(\varepsilon_{3}\left\|U_{m+1, \xi}\right\|_{0, \Omega}^{2}+c\left(\varepsilon_{3}\right)\left\|U_{m+1}\right\|_{0, \Omega}^{2}\right) \\
& +c\left\|U_{m+1}\right\|_{1,2,2, \Omega^{t}}^{2}+c\|\widetilde{F}\|_{0, \Omega^{t}}^{2} \\
& +\varepsilon_{1} \phi(A)\left(\left\|\widetilde{G}_{t}\right\|_{0, S^{t}}^{2}+\sup _{t}\|\widetilde{G}\|_{0, S}^{2}\right) e^{A}+c\left\|U_{m+1}\right\|_{0, \Omega}^{2} .
\end{aligned}
$$

Using (4.43) and (4.44) yields

$$
\begin{aligned}
& \left\|U_{m+1}\right\|_{1, \Omega}^{2}+\left\|U_{m+1, t}\right\|_{0, \Omega^{t}}^{2}+\left\|U_{m+1}\right\|_{1,2,2, \Omega^{t}}^{2} \\
& \leq \phi(A)\left[\varepsilon\left(\left\|\widetilde{G}_{t}\right\|_{0, S^{t}}^{2}+\sup _{t}\|\widetilde{G}\|_{0, S}^{2}\right)\right]+\|\widetilde{F}\|_{0, \Omega^{t}}^{2} \\
& \quad+t \phi(A)\left(\left\|U_{m}\right\|_{2,2,2, \Omega^{t}}^{2}+\sup _{t}\left\|H_{m}\right\|_{1, \Omega}^{2}+\sup _{t}\left\|\Gamma_{m}\right\|_{1, \Omega}^{2}\right) .
\end{aligned}
$$


From the regularity result for the parabolic problem

$$
\begin{array}{ll}
\eta_{m} U_{m+1, t}-\operatorname{div}_{u_{m}} \mathbb{D}_{u_{m}}\left(U_{m+1}\right)=\widetilde{F} & \text { in } \Omega^{T}, \\
\mathbb{D}_{u_{m}}\left(U_{m+1}\right) \cdot \bar{n}_{u_{m}}=\widetilde{G} & \text { on } S^{T}, \\
\left.U_{m+1}\right|_{t=0}=0 & \text { in } \Omega,
\end{array}
$$

we obtain (see Theorem A.1 and Remark A.2 of [6])

$$
\left\|U_{m+1}\right\|_{2,2,2, \Omega^{t}}^{2} \leq c\left(\|\widetilde{F}\|_{0, \Omega^{t}}^{2}+\|\widetilde{G}\|_{1 / 2,2,2, S^{t}}^{2}\right)+c\left\|U_{m+1}\right\|_{0, \Omega^{t}}^{2} .
$$

Using the forms of $\widetilde{F}$ and $\widetilde{G}$ we get

$$
\begin{aligned}
& \|\widetilde{G}\|_{0, S}^{2} \leq t \phi(A)\left(\left\|U_{m}\right\|_{2,2,2, \Omega^{t}}^{2}+\sup _{t}\left\|H_{m}\right\|_{1, \Omega}^{2}+\sup _{t}\left\|\Gamma_{m}\right\|_{1, \Omega}^{2}\right), \\
& \left\|\widetilde{G}_{t}\right\|_{0, S^{t}}^{2} \leq \phi(A)\left(\left\|U_{m}\right\|_{2,2,2, \Omega^{t}}^{2}+\left\|H_{m t}\right\|_{1,2,2, \Omega^{t}}^{2}+\left\|\Gamma_{m t}\right\|_{1,2,2, \Omega^{t}}^{2}\right), \\
& \|\widetilde{G}\|_{1 / 2,2,2, S^{t}}^{2}+\|\widetilde{F}\|_{0, \Omega^{t}}^{2} \\
& \quad \leq t \phi(A)\left(\left\|U_{m}\right\|_{2,2,2, \Omega^{t}}^{2}+\sup _{t}\left\|H_{m}\right\|_{1, \Omega}^{2}+\sup _{t}\left\|\Gamma_{m}\right\|_{1, \Omega}^{2}\right) .
\end{aligned}
$$

From $(4.34)_{7}$ it follows that

$$
\left\|H_{m t}\right\|_{1,2,2, \Omega^{t}}^{2} \leq \phi(A)\left(t \sup _{t}\left\|H_{m}\right\|_{1, \Omega}^{2}+\left\|U_{m}\right\|_{2,2,2, \Omega^{t}}^{2}\right) .
$$

Taking into account (4.45), (4.46)-(4.50) we get

$$
\begin{aligned}
&\left\|U_{m+1}\right\|_{1, \Omega}^{2}+\left\|U_{m+1, t}\right\|_{0, \Omega^{t}}^{2}+\left\|U_{m+1}\right\|_{2,2,2, \Omega^{t}}^{2} \\
& \leq(t+\varepsilon) \phi(A)\left(\left\|U_{m}\right\|_{2,2,2, \Omega^{t}}^{2}+\sup _{t}\left\|H_{m}\right\|_{1, \Omega}^{2}\right. \\
&\left.+\sup _{t}\left\|\Gamma_{m}\right\|_{1, \Omega}^{2}+\left\|\Gamma_{m t}\right\|_{1,2,2, \Omega^{t}}^{2}\right) .
\end{aligned}
$$

Now, integrating $(4.34)_{7}$ with respect to $t$ gives

$$
\begin{aligned}
H_{m}(\xi, t)= & -\exp \left(-\int_{0}^{t} \operatorname{div}_{u_{m}} u_{m} d t^{\prime}\right) \\
& \times \int_{0}^{t}\left[\eta_{m-1}\left(\operatorname{div}_{u_{m}} u_{m}-\operatorname{div}_{u_{m-1}} u_{m-1}\right) \exp \left(\int_{0}^{t^{\prime}} \operatorname{div}_{u_{m}} u_{m} d t^{\prime \prime}\right)\right] d t^{\prime} .
\end{aligned}
$$

Hence

$$
\sup _{t}\left\|H_{m}\right\|_{1, \Omega}^{2} \leq t \phi(A)\left\|U_{m}\right\|_{2,2,2, \Omega^{t}}^{2} .
$$

Using (4.52) and (4.51) we get

$$
\begin{aligned}
& \left\|U_{m+1}\right\|_{1, \Omega}^{2}+\left\|U_{m+1, t}\right\|_{0, \Omega^{t}}^{2}+\left\|U_{m+1}\right\|_{2,2,2, \Omega^{t}}^{2} \\
& \quad \leq(t+\varepsilon) \phi(A)\left(\left\|U_{m}\right\|_{2,2,2, \Omega^{t}}^{2}+\sup _{t}\left\|\Gamma_{m}\right\|_{1, \Omega}^{2}+\left\|\Gamma_{m t}\right\|_{1,2,2, \Omega^{t}}^{2}\right) .
\end{aligned}
$$


In order to estimate $\left\|U_{m+1}\right\|_{1,2,2, \Omega^{t}}^{2}$ we differentiate $(4.34)_{1}$ and $(4.34)_{2}$ with respect to $t$. We obtain

$$
\begin{aligned}
& \eta_{m} U_{m+1, t}-\left[\operatorname{div}_{u_{m}} \mathbb{D}_{u_{m}}\left(U_{m+1}\right]_{, t}=-\eta_{m t} U_{m+1, t}+\widetilde{F}_{t},\right. \\
& {\left[\mathbb{D}_{u_{m}}\left(U_{m+1}\right) \cdot \bar{n}_{u_{m}}\right]_{, t}=\widetilde{G}_{t} .}
\end{aligned}
$$

Multiplying (4.54) by $U_{m+1, t} J_{u_{m}}$, integrating over $\Omega$ and using (4.55) we get

$$
\begin{aligned}
\frac{1}{2} \frac{d}{d t} \int_{\Omega} \eta_{m} & U_{m+1, t}^{2} J_{u_{m}} d \xi+\frac{\mu}{2} \int_{\Omega}\left|\mathbb{S}_{u_{m}}\left(U_{m+1, t}\right)\right|^{2} J_{u_{m}} d \xi \\
& +(\nu-\mu) \int_{\Omega}\left(\operatorname{div}_{u_{m}} U_{m+1, t}\right)^{2} J_{u_{m}} d \xi \\
\leq & \varepsilon\left(\left\|U_{m+1, t}\right\|_{1, \Omega}^{2}+\left\|U_{m+1}\right\|_{2, \Omega}^{2}\right) \\
& +c(\varepsilon)\left\|u_{m}\right\|_{2, \Omega}^{2}\left(\varepsilon\left\|U_{m+1, t}\right\|_{1, \Omega}^{2}+c(\varepsilon)\left\|U_{m+1, t}\right\|_{0, \Omega}^{2}\right) \\
& +c(\varepsilon) t\left\|U_{m+1}\right\|_{2, \Omega}^{2} \sup _{t}\left\|u_{m}\right\|_{2, \Omega}^{2}\left\|u_{m}\right\|_{3,2,2, \Omega^{t}}^{2} \\
& +\int_{\Omega}\left|\widetilde{F}_{t}\right| \cdot\left|U_{m+1, t}\right| \cdot\left|J_{u_{m}}\right| d \xi+\int_{S}\left|\widetilde{G}_{t}\right| \cdot\left|U_{m+1, t}\right| \cdot\left|J_{u_{m}}\right| d \xi_{s} .
\end{aligned}
$$

Now, integrating (4.56) with respect to $t$, using (4.51) and the Korn and Gronwall inequalities we get

$$
\begin{aligned}
\left\|U_{m+1, t}\right\|_{0, \Omega}^{2}+ & \left\|U_{m+1, t}\right\|_{1,2,2, \Omega^{t}}^{2} \\
\leq & (t+\varepsilon) \phi(A)\left(\left\|U_{m}\right\|_{2,2,2, \Omega^{t}}^{2}+\sup _{t}\left\|H_{m}\right\|_{1, \Omega}^{2}\right. \\
& \left.+\left\|H_{m t}\right\|_{1,2,2, \Omega^{t}}^{2}+\sup _{t}\left\|\Gamma_{m}\right\|_{1, \Omega}^{2}+\left\|\Gamma_{m t}\right\|_{1,2,2, \Omega^{t}}^{2}\right) .
\end{aligned}
$$

Collecting (4.50), (4.52), (4.53) and (4.57) yields

$$
\begin{gathered}
\left\|U_{m+1}\right\|_{1, \Omega}^{2}+\left\|U_{m+1, t}\right\|_{0, \Omega}^{2}+\left\|U_{m+1}\right\|_{2,2,2, \Omega^{t}}^{2}+\left\|U_{m+1, t}\right\|_{1,2,2, \Omega^{t}}^{2} \\
\leq(t+\varepsilon) \phi(A)\left(\left\|U_{m}\right\|_{2,2,2, \Omega^{t}}^{2}+\sup _{t}\left\|U_{m}\right\|_{1, \Omega}^{2}\right. \\
\left.+\sup _{t}\left\|\Gamma_{m}\right\|_{1, \Omega}^{2}+\left\|\Gamma_{m t}\right\|_{1,2,2, \Omega^{t}}^{2}\right) .
\end{gathered}
$$

It remains to find estimates for $\left\|\Gamma_{m+1}\right\|_{0, \Omega}^{2}+\left\|\Gamma_{m+1, t}\right\|_{0, \Omega}^{2}+\left\|\Gamma_{m+1}\right\|_{2,2,2, \Omega^{t}}^{2}$ $+\left\|\Gamma_{m+1, t}\right\|_{1,2,2, \Omega^{t}}^{2}$. First, we multiply (4.34) $)_{4}$ by $\Gamma_{m+1} J_{u_{m}}$ and integrate over $\Omega$ to obtain

$$
\begin{array}{r}
\frac{1}{2} \int_{\Omega} \eta_{m} c_{v}\left(\eta_{m}, \gamma_{m}\right) \frac{d}{d t} \Gamma_{m+1}^{2} J_{u_{m}} d \xi+\kappa \int_{\Omega}\left|\nabla_{u_{m}} \Gamma_{m+1}\right|^{2} J_{u_{m}} d \xi \\
=\int_{\Omega} \widetilde{I} \Gamma_{m+1} J_{u_{m}} d \xi+\int_{S} \widetilde{J} \Gamma_{m+1} J_{u_{m}} d \xi_{s}
\end{array}
$$


where

(4.60) $\int_{\Omega^{t}}\left|\widetilde{I} \Gamma_{m+1} J_{u_{m}}\right| d \xi d t^{\prime} \leq \varepsilon\left\|\Gamma_{m+1}\right\|_{1,2,2, \Omega^{t}}^{2}+t \phi(A)\left(\left\|U_{m}\right\|_{2,2,2, \Omega^{t}}^{2}\right.$

$$
\left.+\sup _{t}\left\|U_{m}\right\|_{1, \Omega}^{2}+\sup _{t}\left\|H_{m}\right\|_{1, \Omega}^{2}+\sup _{t}\left\|\Gamma_{m}\right\|_{1, \Omega}^{2}\right)
$$

and

$$
\int_{S^{t}}\left|\widetilde{J} \Gamma_{m+1} J_{u_{m}}\right| d \xi_{s} d t^{\prime} \leq \varepsilon\left\|\Gamma_{m+1}\right\|_{1,2,2, \Omega^{t}}^{2}+t \phi(A)\left\|U_{m}\right\|_{2,2,2, \Omega^{t}}^{2} .
$$

Integrating (4.59) with respect to $t$, using (4.60)-(4.61), (4.52) and the Gronwall inequality yields

$$
\begin{aligned}
\left\|\Gamma_{m+1}\right\|_{0, \Omega}^{2}+\left\|\Gamma_{m+1}\right\|_{1,2,2, \Omega^{t}}^{2} & \\
& \leq t \phi(A)\left(\left\|U_{m}\right\|_{2,2,2, \Omega^{t}}^{2}+\sup _{t}\left\|U_{m}\right\|_{1, \Omega}^{2}+\sup _{t}\left\|\Gamma_{m}\right\|_{1, \Omega}^{2}\right) .
\end{aligned}
$$

Next, multiplying (4.34) $)_{4}$ by $\Gamma_{m+1, t} J_{u_{m}}$ and integrating over $\Omega$ we get

$$
\begin{array}{r}
\int_{\Omega} \eta_{m} c_{v}\left(\eta_{m}, \gamma_{m}\right)\left|\Gamma_{m+1, t}\right|^{2} J_{u_{m}} d \xi+\kappa \int_{\Omega} \nabla_{u_{m}} \Gamma_{m+1} \cdot \nabla_{u_{m}} \Gamma_{m+1, t} J_{u_{m}} d \xi \\
=\int_{S} \widetilde{J} \Gamma_{m+1, t} J_{u_{m}} d \xi_{s}+\int_{\Omega} \widetilde{I} \Gamma_{m+1, t} J_{u_{m}} d \xi .
\end{array}
$$

Hence

$$
\begin{aligned}
\int_{\Omega} \eta_{m} c_{v}\left(\eta_{m}, \gamma_{m}\right) \mid & \left.\Gamma_{m+1, t}\right|^{2} J_{u_{m}} d \xi \\
& +\kappa \int_{\Omega} \nabla_{u_{m}} \Gamma_{m+1} \frac{d}{d t}\left(\nabla_{u_{m}} \Gamma_{m+1}\right) J_{u_{m}} d \xi \\
& -\kappa \int_{\Omega} \nabla_{u_{m}} \Gamma_{m+1} \frac{d}{d t}\left(\nabla_{u_{m}}\right) \cdot \Gamma_{m+1} J_{u_{m}} d \xi \\
= & \frac{d}{d t} \int_{S} \widetilde{J} \Gamma_{m+1} J_{u_{m}} d \xi_{s}-\int_{S} \widetilde{J_{t}} \Gamma_{m+1} J_{u_{m}} d \xi_{s} \\
& -\int_{S} \widetilde{J} \Gamma_{m+1} \operatorname{div}_{u_{m}} u_{m} J_{u_{m}} d \xi_{s}+\int_{\Omega} \widetilde{I} \Gamma_{m+1, t} J_{u_{m}} d \xi .
\end{aligned}
$$

From (4.63) it follows that

$$
\begin{aligned}
& \int_{\Omega} \eta_{m} c_{v}\left(\eta_{m}, \gamma_{m}\right)\left|\Gamma_{m+1, t}\right|^{2} J_{u_{m}} d \xi+\kappa \frac{d}{d t} \int_{\Omega}\left|\nabla_{u_{m}} \Gamma_{m+1}\right|^{2} J_{u_{m}} d \xi \\
& \leq \frac{d}{d t} \int_{S} \widetilde{J} \Gamma_{m+1} J_{u_{m}} d \xi_{s}+\varepsilon_{1}\left\|\widetilde{J}_{t}\right\|_{0, S}^{2}+c\left(\varepsilon_{1}\right)\left\|\Gamma_{m+1}\right\|_{1, \Omega}^{2} \\
& \quad+\varepsilon\left\|\Gamma_{m+1, t}\right\|_{0, \Omega}^{2}+c(\varepsilon)\|\widetilde{I}\|_{0, \Omega}^{2}+\varepsilon_{1}\left\|u_{m}\right\|_{2, \Omega}^{2}\left\|J_{u_{m}}\right\|_{2, \Omega}^{2}\|\widetilde{J}\|_{0, S}^{2} \\
& \quad+c\left\|u_{m}\right\|_{3, \Omega}^{2} \int_{\Omega}\left|\nabla_{u_{m}} \Gamma_{m+1}\right|^{2} J_{u_{m}} d \xi .
\end{aligned}
$$


Integrating (4.64) with respect to $t$ and using the Gronwall inequality yields

$$
\begin{aligned}
\left\|\Gamma_{m+1, t}\right\|_{0, \Omega^{t}}^{2}+\left\|\Gamma_{m+1}\right\|_{1, \Omega}^{2} & \\
\leq & {\left[\varepsilon_{2} \sup _{t}\|\widetilde{J}\|_{0, S}^{2}+c\left(\varepsilon_{2}\right)\left(\varepsilon_{3}\left\|\Gamma_{m+1, \xi}\right\|_{0, \Omega}^{2}+c\left(\varepsilon_{3}\right)\left\|\Gamma_{m+1}\right\|_{0, \Omega}^{2}\right)\right.} \\
& +c\left(\varepsilon_{1}\right)\left\|\Gamma_{m+1}\right\|_{1,2,2, \Omega^{t}}^{2}+\varepsilon\left\|\Gamma_{m+1, t}\right\|_{0, \Omega}^{2}+c(\varepsilon)\|\widetilde{I}\|_{0, \Omega^{t}}^{2} \\
& \left.+\varepsilon_{1}\left(\left\|\widetilde{J}_{t}\right\|_{0, S^{t}}^{2}+\phi(A) \sup _{t}\|\widetilde{J}\|_{0, S}^{2}\right)\right] e^{A} .
\end{aligned}
$$

Now, from the regularity result for the parabolic problem

$$
\begin{array}{ll}
\eta_{m} c_{v}\left(\eta_{m}, \gamma_{m}\right) \Gamma_{m+1, t}-\kappa \nabla_{u_{m}}^{2} \Gamma_{m+1}=\widetilde{I} & \text { in } \Omega^{T}, \\
\bar{n}\left(u_{m}\right) \cdot \nabla_{u_{m}} \Gamma_{m+1}=\widetilde{J} & \text { on } S^{T}, \\
\left.\Gamma_{m+1}\right|_{t=0}=0 & \text { in } \Omega,
\end{array}
$$

we obtain as before (see Theorem A.1 and Remark A.2 of [6])

$$
\left\|\Gamma_{m+1}\right\|_{2,2,2, \Omega^{t}}^{2} \leq c\left(\|\widetilde{I}\|_{0, \Omega^{t}}^{2}+\|\widetilde{J}\|_{1 / 2,2,2, S^{t}}^{2}+\left\|\Gamma_{m+1}\right\|_{0, \Omega^{t}}^{2}\right) .
$$

Taking into account inequalities (4.62), (4.65) and (4.66) we get

$$
\begin{aligned}
\left\|\Gamma_{m+1}\right\|_{1, \Omega}^{2}+\left\|\Gamma_{m+1, t}\right\|_{0, \Omega^{t}}^{2}+\left\|\Gamma_{m+1}\right\|_{2,2,2, \Omega^{t}}^{2} & \\
\leq & \phi(A)\left(\varepsilon_{1}\left\|\widetilde{J}_{t}\right\|_{0, S^{t}}^{2}+\varepsilon_{1} \sup _{t}\|\widetilde{J}\|_{0, S}^{2}+\|\widetilde{I}\|_{0, \Omega^{t}}^{2}+\|\widetilde{J}\|_{1 / 2,2,2, S^{t}}^{2}\right) \\
& +t \phi(A)\left(\left\|U_{m}\right\|_{2,2,2, \Omega^{t}}^{2}+\sup _{t}\left\|U_{m}\right\|_{1, \Omega}^{2}+\sup _{t}\left\|\Gamma_{m}\right\|_{1, \Omega}^{2}\right) .
\end{aligned}
$$

We estimate the terms on the r.h.s. of (4.67). We have

$$
\begin{aligned}
&\|\widetilde{I}\|_{0, \Omega^{t}}^{2} \leq t \phi(A)\left(\left\|U_{m}\right\|_{2,2,2, \Omega^{t}}^{2}+\sup _{t}\left\|U_{m}\right\|_{1, \Omega}^{2}+\sup _{t}\left\|\Gamma_{m}\right\|_{1, \Omega}^{2}\right) \\
&+\left\|I_{3}\right\|_{0, \Omega^{t}}^{2}+\left\|I_{4}\right\|_{0, \Omega^{t}}^{2},
\end{aligned}
$$

where

$$
\begin{aligned}
\left\|I_{3}\right\|_{0, \Omega^{t}}^{2}+\left\|I_{4}\right\|_{0, \Omega^{t}}^{2} \leq & c\left(\int_{\Omega^{t}}\left|u_{m \xi}\right|^{2}\left|\int_{0}^{t^{\prime}} U_{m \xi} d t^{\prime \prime}\right|^{2}\left|u_{m \xi}\right|^{2} d \xi d t^{\prime}\right. \\
& +\int_{\Omega^{t}}\left|u_{m \xi}\right|^{2}\left|\int_{0}^{t^{\prime}} U_{m \xi t} d t^{\prime \prime}\right|^{2} d \xi d t^{\prime} \\
& +\int_{\Omega^{t}}\left|u_{m-1, \xi}\right|^{2}\left|\int_{0}^{t^{\prime}} U_{m \xi t} d t^{\prime \prime}\right|^{2} d \xi d t^{\prime} \\
& \left.+\int_{\Omega^{t}}\left|u_{m-1, \xi}\right|^{2}\left|\int_{0}^{t^{\prime}} U_{m \xi} d t^{\prime \prime}\right|^{2}\left|u_{m \xi}\right|^{2} d \xi d t^{\prime}\right) \\
\leq & t \phi(A)\left(\left\|U_{m}\right\|_{2,2,2, \Omega^{t}}^{2}+\left\|U_{m t}\right\|_{1,2,2, \Omega^{t}}^{2}\right) .
\end{aligned}
$$


Next,

$$
\begin{gathered}
\sup _{t}\|\widetilde{J}\|_{0, S}^{2} \leq t \phi(A)\left\|U_{m}\right\|_{2,2,2, \Omega^{t}}^{2}, \\
\left\|\widetilde{J_{t}}\right\|_{0, S^{t}}^{2} \leq(t+1) \phi(A)\left\|U_{m}\right\|_{2,2,2, \Omega^{t}}^{2}, \\
\|\widetilde{J}\|_{1 / 2,2,2, S^{t}}^{2} \leq t \phi(A)\left\|U_{m}\right\|_{2,2,2, \Omega^{t}}^{2} .
\end{gathered}
$$

Collecting inequalities (4.67)-(4.72) gives

$$
\begin{aligned}
\left\|\Gamma_{m+1}\right\|_{1, \Omega}^{2}+\| \Gamma_{m+1, t} & \left\|_{0, \Omega^{t}}^{2}+\right\| \Gamma_{m+1} \|_{2,2,2, \Omega^{t}}^{2} \\
\leq & \left(\varepsilon_{1}+t\right) \phi(A)\left(\left\|U_{m}\right\|_{2,2,2, \Omega^{t}}^{2}+\sup _{t}\left\|U_{m}\right\|_{1, \Omega}^{2}\right. \\
& \left.+\left\|U_{m t}\right\|_{1,2,2, \Omega^{t}}^{2}+\sup _{t}\left\|\Gamma_{m}\right\|_{1, \Omega}^{2}\right) .
\end{aligned}
$$

Finally, we obtain an estimate for $\left\|\Gamma_{m+1, t}\right\|_{1,2,2, \Omega^{t}}^{2}$. After differentiating $(4.34)_{4}$ with respect to $t$ we get

$$
\begin{gathered}
\eta_{m} c_{v}\left(\eta_{m}, \gamma_{m}\right) \Gamma_{m+1, t t}-\kappa\left(\nabla_{u_{m}}^{2} \Gamma_{m+1}\right)_{, t} \\
=-\eta_{m t} c_{v}\left(\eta_{m}, \gamma_{m}\right) \Gamma_{m+1, t}-\eta_{m}\left(c_{v \eta} \eta_{m t}+c_{v \gamma} \gamma_{m t}\right) \Gamma_{m+1, t}+\widetilde{I}_{t}, \\
\left(\bar{n}_{u_{m}} \cdot \nabla_{u_{m}} \Gamma_{m+1}\right)_{, t}=\widetilde{J}_{t} .
\end{gathered}
$$

Multiplying (4.74) by $\Gamma_{m+1, t} J_{u_{m}}$, integrating over $\Omega^{t}$, using the boundary condition (4.75) and next the Gronwall inequality and estimates (4.73), (4.50), (4.52) yields

$$
\begin{gathered}
\left\|\Gamma_{m+1}\right\|_{1, \Omega}^{2}+\left\|\Gamma_{m+1, t}\right\|_{0, \Omega^{t}}^{2}+\left\|\Gamma_{m+1, t}\right\|_{1,2,2, \Omega^{t}}^{2}+\left\|\Gamma_{m+1}\right\|_{2,2,2, \Omega^{t}}^{2} \\
\leq(\varepsilon+t) \phi(A)\left(\left\|U_{m}\right\|_{2,2,2, \Omega^{t}}^{2}+\sup _{t}\left\|U_{m}\right\|_{1, \Omega}^{2}\right. \\
\left.+\left\|U_{m t}\right\|_{1,2,2, \Omega^{t}}^{2}+\sup _{t}\left\|\Gamma_{m}\right\|_{1, \Omega}^{2}\right) .
\end{gathered}
$$

Adding (4.58) and (4.76) we obtain

$$
\begin{aligned}
\sup _{t}\left\|U_{m+1}\right\|_{1, \Omega}^{2} & +\sup _{t}\left\|\Gamma_{m+1}\right\|_{1, \Omega}^{2}+\left\|U_{m+1, t}\right\|_{0, \Omega^{t}}^{2} \\
& +\left\|\Gamma_{m+1, t}\right\|_{0, \Omega^{t}}^{2}+\left\|U_{m+1}\right\|_{2,2,2, \Omega^{t}}^{2}+\left\|\Gamma_{m+1}\right\|_{2,2,2, \Omega^{t}}^{2} \\
& +\left\|U_{m+1, t}\right\|_{1,2,2, \Omega^{t}}^{2}+\left\|\Gamma_{m+1, t}\right\|_{1,2,2, \Omega^{t}}^{2} \\
\leq & (\varepsilon+t) \phi(A)\left(\left\|U_{m}\right\|_{2,2,2, \Omega^{t}}^{2}++\sup _{t}\left\|U_{m}\right\|_{1, \Omega}^{2}\right. \\
& \left.+\left\|U_{m t}\right\|_{1,2,2, \Omega^{t}}^{2}+\left\|\Gamma_{m t}\right\|_{1,2,2, \Omega^{t}}^{2}+\sup _{t}\left\|\Gamma_{m}\right\|_{1, \Omega}^{2}\right) .
\end{aligned}
$$

Assume that $T_{*}>0$ from Lemma 4.1 is so small that

$$
\phi(A)\left(T_{*}+\varepsilon\right)<1 .
$$


Then for $t \leq T$ (where $T \leq T_{*}$ ) estimate (4.77) implies the strong convergence $\left(u_{m}, \gamma_{m}, \eta_{m}\right)$ to a solution $(u, \gamma, \eta)$ of problem (1.4) and $u, \gamma \in \mathcal{A}_{T}$, $\eta \in \mathcal{B}_{T}$. Moreover, inequality (4.13) implies (4.32) and by Lemma 2.3 we get (4.33).

The uniqueness follows for sufficiently small $T_{*}$ from the inequality

$$
\begin{aligned}
\sup _{t}\|U\|_{1, \Omega}^{2}+ & \sup _{t}\|\Gamma\|_{1, \Omega}^{2}+\left\|U_{t}\right\|_{0, \Omega^{t}}^{2}+\left\|\Gamma_{t}\right\|_{0, \Omega^{t}}^{2} \\
& +\|U\|_{2,2,2, \Omega^{t}}^{2}+\|\Gamma\|_{2,2,2, \Omega^{t}}^{2}+\left\|U_{t}\right\|_{1,2,2, \Omega^{t}}^{2}+\left\|\Gamma_{t}\right\|_{1,2,2, \Omega^{t}}^{2} \\
\leq & (\varepsilon+t) \phi(A)\left(\|U\|_{2,2,2, \Omega^{t}}^{2}+\sup _{t}\|U\|_{1, \Omega}^{2}+\left\|U_{t}\right\|_{1,2,2, \Omega^{t}}^{2}\right. \\
& \left.+\left\|\Gamma_{t}\right\|_{1,2,2, \Omega^{t}}^{2}+\sup _{t}\|\Gamma\|_{1, \Omega}^{2}\right)
\end{aligned}
$$

(where $U=u_{1}-u_{2}, \Gamma=\gamma_{1}-\gamma_{2} ;\left(u_{1}, \gamma_{1}\right),\left(u_{2}, \gamma_{2}\right)$ are two solutions of (1.4)), which can be obtained in the same way as (4.77).

This completes the proof of the theorem.

\section{References}

[1] O. V. Besov, V. P. Il'in and S. M. Nikol'skiŭ, Integral Representations of Functions and Imbedding Theorems, Nauka, Moscow, 1986 (in Russian).

[2] B. Ducomet, Hydrodynamical models od gaseous stars, Rep. Math. Phys., to appear.

[3] L. Landau and E. Lifschitz, Hydrodynamics, Nauka, Moscow, 1986 (in Russian); English transl.: Fluid Mechanics, Pergamon Press, Oxford, 1987.

[4] P. Secchi, On the motion of gaseous stars in presence of radiation, Comm. Partial Differential Equations 15 (1990), 185-204.

[5] V. A. Solonnikov, On boundary value problems for linear parabolic systems of differential equations of general type, Trudy Mat. Inst. Steklov. 83 (1965) (in Russian); English transl.: Proc. Steklov Inst. Math. 83 (1967).

[6] G. Ströhmer and W. M. Zajạczkowski, Local existence of solutions of free boundary problem for the equations of compressible barotropic viscous self-gravitating fluids, to appear.

[7] E. Zadrzyńska and W. M. Zajączkowski, On local motion of a general compressible viscous heat conducting fluid bounded by a free surface, Ann. Polon. Math. 59 (1994), 133-170.

[8] - - - Conservation laws in free boundary problems for viscous compressible heatconducting fluids, Bull. Polish Acad. Sci. Tech. Sci. 42 (1994), 197-207.

[9] - - - On a differential inequality for equations of a viscous compressible heat conducting fluid bounded by a free surface, Ann. Polon. Math. 61 (1995), 141-188.

[10] - - - On the global existence theorem for a free boundary problem for equations of a viscous compressible heat conducting fluid, ibid. 63 (1996), 199-221.

[11] - - - On global motion of a compressible viscous heat conducting fluid bounded by a free surface, Acta Appl. Math. 37 (1994), 221-231. 
[12] E. Zadrzyńska and W. M. Zajączkowski, On nonstationary motion of a fixed mass of a viscous compressible barotropic fluid bounded by a free boundary, in preparation.

Ewa Zadrzyńska

Institute of Mathematics and Operations Research Military University of Technology

S. Kaliskiego 2

01-489 Warszawa, Poland

E-mail: e.zadrzynska@imbo.wat.waw.pl
Wojciech M. Zajączkowski

Institute of Mathematics Polish Academy of Sciences Śniadeckich 8

00-950 Warszawa, Poland E-mail: wmzajacz@impan.gov.pl

Received on 1.4.1997 